JOURNAL OF THE

AMERICAN MATHEMATICAL SOCIETY

Volume 11, Number 4, October 1998, Pages 1001-1036

S 0894-0347(98)00272-0

\title{
UNE COMPACTIFICATION DES CHAMPS CLASSIFIANT LES CHTOUCAS DE DRINFELD
}

\author{
LAURENT LAFFORGUE
}

\section{SOMMAIRE}

Introduction

1. Le champ des chtoucas itérés. Algébricité. Lissité. Propreté

a) Le schéma des homomorphismes complets

b) Le champ des pré-chtoucas itérés

c) Description des différentes strates. Le champ des chtoucas itérés

d) Sous-objets. Troncatures

2. Vérification du critère valuatif de propreté

a) $\varphi$-réseaux itérés dans les $\varphi$-espaces

b) Chtoucas dégénérés associés aux $\varphi$-réseaux itérés

c) Transformations des chtoucas dégénérés

d) Critères d'existence de telles transformations

e) Dégénérescence des chtoucas en chtoucas itérés

Bibliographie

\section{INTRODUCTION}

Etant donnée $X$ une courbe projective lisse géométriquement connexe au-dessus d'un corps fini $\mathbb{F}_{q}$, Drinfeld a défini la notion de chtouca de rang $r$. Leurs champs classifiants $\mathrm{Cht}^{r}$ sont algébriques au sens de Deligne-Mumford, localement de type fini et lisses de dimension relative $2 r-2$ au-dessus de $X \times X$. Mais les composantes connexes de ces champs ne sont pas propres ni même de type fini dès que $r \geq 2$. Quand $r=2$, Drinfeld a également construit des compactifications modulaires lisses pour les ouverts de type fini de $\mathrm{Cht}^{2}$, ce qui constitue l'un des pas de sa démonstration de la correspondance de Langlands en rang 2 sur les corps de fonctions.

Dans ce travail, nous proposons une généralisation en rang quelconque de la construction par Drinfeld de ces compactifications.

Rappelons qu'un chtouca (à droite) de rang $r$ sur un schéma de base $S\left(\operatorname{sur} \mathbb{F}_{q}\right.$ ) consiste en la donnée de

- un fibré $\mathcal{E}$ localement libre de $\operatorname{rang} r$ sur $X \times S$,

- une modification (à droite) de $\mathcal{E}$, c'est-à-dire un diagramme $\mathcal{E} \stackrel{j}{\longleftrightarrow} \mathcal{E}^{\prime} \stackrel{t}{\longleftrightarrow}$ $\mathcal{E}^{\prime \prime}$ où $\mathcal{E}^{\prime}, \mathcal{E}^{\prime \prime}$ sont aussi des fibrés localement libres de $\operatorname{rang} r$ sur $X \times S$, et $j, t$ sont

Received by the editors June 9, 1997 and, in revised form, March 30, 1998.

1991 Mathematics Subject Classification. Primary 11R58, 11G09, 14G35.

Key words and phrases. Corps de fonctions, champs modulaires de Drinfeld, chtoucas.

(C)1998 American Mathematical Society 
des homomorphismes injectifs dont les conoyaux sont supportés par les graphes de deux morphismes "pôle" et "zéro" de $S$ dans $X$ et inversibles comme $\mathcal{O}_{S}$-Modules, - un isomorphisme ${ }^{\tau} \mathcal{E}=\left(\operatorname{Id}_{X} \times \operatorname{Frob}_{S}\right)^{*} \mathcal{E} \stackrel{\sim}{\longrightarrow} \mathcal{E}^{\prime \prime}$.

L'idée est d'élargir cette définition en remplaçant dans le dernier point la notion d'isomorphisme par celle plus souple d' "homomorphisme complet". Les "homomorphismes complets" sont essentiellement les morphismes à valeurs dans le schéma obtenu en éclatant dans le schéma matriciel $M_{r} \backslash\{0\}$ les fermés des homomorphismes de rang $\leq 1$, de rang $\leq 2$, etc. (voir [5] par exemple). A noter que le quotient de ce schéma éclaté par l'action de $\mathbb{G}_{m}$ n'est autre que le compactifié de $\left(\mathrm{PGL}_{r} \times \mathrm{PGL}_{r}\right) / \mathrm{PGL}_{r}$ à la façon de De Concini et Procesi.

Le champ $\overline{\mathrm{Cht}^{r}}$ classifiant ces nouveaux objets est algébrique au sens de DeligneMumford et localement de type fini sur $X \times X$; il contient $\mathrm{Cht}^{r}$ comme ouvert et il vérifie la partie d'existence (mais non d'unicité : il n'est pas séparé) du critère valuatif de propreté. Il se décompose en strates localement fermées $\overline{\mathrm{Cht}_{\underline{r}}^{r}}$ indexées par les partitions $\underline{r}=\left(0=r_{0}<r_{1}<\cdots<r_{k}=r\right)$ de l'entier $r$; chacune classifie essentiellement les familles de chtoucas de rangs $r_{1}, r_{2}-r_{1}, \ldots, r-r_{k-1}$ telles que le zéro de chacun soit égal au pôle du suivant ; toute telle strate est donc munie d'un morphisme lisse sur $X \times X \times X^{k-1}$, où le facteur $X^{k-1}$ généralise le "dégénérateur" introduit par Drinfeld quand $r=2$ et $\underline{r}=(0<1<2)$. C'est aussi pourquoi on appellera $\overline{\text { Cht }^{r}}$ le champ des chtoucas itérés de rang $r$.

On sait que le champ $\mathrm{Cht}^{r}$ est réunion filtrante des ouverts $\mathrm{Cht}^{r, \bar{p}} \leq p$ classifiant les chtoucas dont le polygone canonique de Harder-Narasimhan est majoré par un polygone de troncature $p:[0, r] \rightarrow \mathbb{R}$. Et chaque $\mathrm{Cht}^{r, \bar{p}} \leq p$ est réunion disjointe de champs de type fini. Quand $p$ est assez grand, on définit dans le fourre-tout $\overline{\mathrm{Cht}^{r}}$ un ouvert $\overline{\mathrm{Cht}^{r}, \bar{p} \leq p}$ dont la trace dans $\mathrm{Cht}^{r}$ est égale à $\mathrm{Cht}^{r}, \bar{p} \leq p$ et qui est réunion disjointe de champs propres et lisses (en particulier séparés et de type fini) sur $X \times X$.

Signalons que l'auteur compte exposer dans un prochain article la construction de semblables compactifications modulaires pour les chtoucas avec structures de niveau. Le présent texte donne les démonstrations de résultats annoncés dans une note aux Comptes Rendus de l'Académie des Sciences de Paris (tome 323, série I, pp. 491-494). En voici le contenu:

Le paragraphe 1 décrit les étapes successives de la construction du champ $\overline{\mathrm{Cht}^{r}}$ des chtoucas itérés de rang $r$, avec ses strates $\overline{\mathrm{Cht}_{\underline{r}}^{r}}$ et ses ouverts $\overline{\mathrm{Cht}^{r}, \bar{p} \leq p}$, ainsi que ses principales propriétés géométriques.

En a) on rappelle la définition du schéma $\Omega^{r}$ des "homomorphismes complets", sa stratification naturelle et la description modulaire de ses strates $\Omega_{\underline{r}}^{r}$.

En b) on substitue les "homomorphismes complets" aux isomorphismes dans les données de définition des chtoucas pour obtenir un nouveau champ $\mathcal{C}^{r}$, dit des pré-chtoucas itérés de rang $r$, qui est algébrique au sens d'Artin.

En c) on déduit de la stratification $\left(\Omega_{r}^{r}\right)$ de $\Omega^{r}$ une stratification $\left(\mathcal{C}_{r}^{r}\right)$ de $\mathcal{C}^{r}$. La description modulaire de chaque strate $\bar{\Omega}_{\underline{r}}^{r}$ induit une description modulaire de $\mathcal{C}_{\underline{r}}^{r}$ qui devient particulièrement simple dans un certain ouvert $\overline{\mathrm{Cht}_{\underline{r}}^{r}}$ de $\mathcal{C}_{\underline{\underline{r}}}^{r}$. Les ouverts $\overline{\mathrm{Cht}_{r}^{r}}$ des $\mathcal{C}_{r}^{r}$ proviennent d'un ouvert $\overline{\mathrm{Cht}^{r}}$ de $\mathcal{C}^{r}$, qu'on appelle champ des chtoucas itérés de rang $r$; il est algébrique au sens de Deligne-Mumford.

En d) on définit pour tout polygone de troncature $p:[0, r] \rightarrow \mathbb{R}$ un ouvert $\overline{\mathrm{Cht}^{r}, \bar{p} \leq p}$ de $\overline{\mathrm{Cht}^{r}}$ dont la trace dans $\mathrm{Cht}^{r}$ est égale à $\mathrm{Cht}^{r, \bar{p}} \leq p$. On prouve qu'il est 
lisse de dimension $2 r-2$ sur $X \times X$ si $p$ est assez grand et que ses composantes connexes sont de type fini. Afin de montrer que celles-ci sont propres, il suffira donc de vérifier le critère valuatif de propreté pour $\overline{\mathrm{Cht}^{r, \bar{p}} \leq p}$.

C'est l'objet du paragraphe 2. On procède à la manière de Drinfeld en rang 2. On se donne donc $\widetilde{\mathcal{E}}$ un point de $\mathrm{Cht}^{r, \bar{p} \leq p}$ à valeurs dans le corps des fractions $K_{A}$ d'un anneau de valuation discrète $A$ et on cherche à prouver que (quitte à remplacer $A$ par une extension finie normale) $\widetilde{\mathcal{E}}$ se prolonge de manière unique en un point de $\overline{\mathrm{Cht}^{r,}, \bar{p} \leq p}$ à valeurs dans $A$. Un tel prolongement va consister en la donnée de certains diagrammes de fibrés sur $X \otimes A$, donnée équivalente à celle d'un réseau $M$ dans la fibre générique $V$ de $\widetilde{\mathcal{E}}$.

En a) on introduit certaines premières propriétés que devra vérifier le réseau $M$ et on montre l'existence de réseaux les vérifiant, appelés " $\varphi$-réseaux itérés".

En b) on explicite comment les $\varphi$-réseaux itérés induisent des diagrammes de fibrés prolongés sur $X \otimes A$, qu'on appelle "chtoucas dégénérés".

En c) on introduit certaines opérations de transformation des $\varphi$-réseaux itérés et on étudie leurs effets sur les chtoucas dégénérés associés.

En d) on donne des conditions suffisantes pour que les opérations de c) soient possibles.

En e) enfin, on démontre le critère valuatif de propreté comme annoncé. Pour la partie d'existence, on part d'un $\varphi$-réseau itéré dont l'existence a été assurée en a) et on lui fait subir une série de transformations selon les procédés de c) et d); après un nombre fini de pas, on obtient un $\varphi$-réseau itéré dont le chtouca dégénéré associé vérifie toutes les propriétés requises.

Je remercie profondément Gérard Laumon pour sa disponibilité jamais lassée face à mes question mathématiques et pour son soutien moral constant.

Je tiens à exprimer aussi ma gratitude envers Vladimir Drinfeld dont l'encouragement à chercher des compactifications modulaires lisses fut pour moi décisif.

Enfin, j'adresse mes plus vifs remerciements à Mme Le Bronnec qui a assuré avec compétence et patience la saisie du manuscrit.

1. Le Champ des chtoucas itérés. Algébricité. Lissité. Propreté

a) Le schéma des homomorphismes complets. Ce paragraphe rappelle une construction connue. On renvoie par exemple à [5] pour une revue générale.

Soit $r \geq 1$ un nombre entier.

Soit $H^{r}$ le foncteur qui associe à tout anneau $A$ le module des $\left(u_{1}, u_{2}, \ldots, u_{r}\right.$; $\left.\alpha_{1}, \alpha_{2}, \ldots, \alpha_{r-1}\right) \in \operatorname{Hom}\left(A^{r}, A^{r}\right) \times \operatorname{Hom}\left(\Lambda^{2} A^{r}, \Lambda^{2} A^{r}\right) \times \cdots \times \operatorname{Hom}\left(\Lambda^{r} A^{r}, \Lambda^{r} A^{r}\right) \times$ $A^{r-1}$. Ce foncteur est représentable par un fibré vectoriel libre de rang fini audessus de Spec $\mathbb{Z}$.

Il contient comme sous-schéma localement fermé le schéma $\Omega_{(r)}^{r}$ classifiant les $\left(u_{1}, u_{2}, \ldots, u_{r} ; \alpha_{1}, \alpha_{2}, \ldots, \alpha_{r-1}\right)$ tels que $u_{1}$ soit un automorphisme, que $\alpha_{1}$, $\alpha_{2}, \ldots, \alpha_{r-1}$ soient inversibles et que $\Lambda^{2} u_{1}=\alpha_{1} u_{2}, \Lambda^{3} u_{1}=\alpha_{1}^{2} \alpha_{2} u_{3}, \ldots, \Lambda^{r} u_{1}=$ $\alpha_{1}^{r-1} \alpha_{2}^{r-2} \cdots \alpha_{r-1} u_{r}$.

Soit $\Omega^{r}$ l'adhérence schématique de $\Omega_{(r)}^{r}$ dans l'ouvert de $H^{r}$ défini par la condition que $u_{1}, u_{2}, \ldots, u_{r}$ soient partout non nuls. On voit déjà que $\Omega^{r}$ est plat et de type fini sur Spec $\mathbb{Z}$, de dimension relative $r^{2}+(r-1)$ et muni d'un morphisme $\left(\alpha_{1}, \alpha_{2}, \ldots, \alpha_{r-1}\right): \Omega^{r} \rightarrow \mathbb{A}^{r-1}$.

Pour toute partition de $r$, c'est-⿳亠㐅े-dire toute famille $\underline{r}=\left(r_{1}, \ldots, r_{k}\right)$ vérifiant $0<r_{1}<\cdots<r_{k}=r$ (et que l'on pourra compléter en posant $r_{0}=0$ ), soit $\Omega_{\underline{r}}^{r}$ 
le sous-schéma localement fermé de $\Omega^{r}$ défini par la condition que chaque $\alpha_{s}$ soit partout nul si $s \in \underline{r}$ et partout non nul si $s \notin \underline{r}$.

Proposition 1. Pour toute partition $\underline{r}=\left(r_{1}, \ldots, r_{k}\right)$ comme ci-dessus, le schéma $\Omega_{\underline{r}}^{r}$ représente le foncteur qui à tout anneau $A$ associe l'ensemble des uplets

$$
\left(v_{1}, v_{2}, \ldots, v_{k} ; \alpha_{1}, \ldots, \alpha_{r_{1}-1}, \alpha_{r_{1}+1}, \ldots, \alpha_{r_{2}-1}, \alpha_{r_{2}+1}, \ldots\right)
$$

tels que:

- les $\alpha_{s}, s \notin \underline{r}$, sont des scalaires partout inversibles,

- $v_{1}: A^{r} \rightarrow A^{r}$ est un homomorphisme de rang partout égal à $r_{1}$,

- $v_{2}: \operatorname{Ker} v_{1} \rightarrow A^{r} / \operatorname{Im} v_{1}$ est un homomorphisme de rang partout égal à $r_{2}-r_{1}$,

- $v_{3}: \operatorname{Ker} v_{2} \rightarrow\left(A^{r} / \operatorname{Im} v_{1}\right) / \operatorname{Im} v_{2}$ est un homomorphisme de rang partout égal $\grave{a} r_{3}-r_{2}$,

- etc.

A fortiori, le morphisme $\left(\alpha_{1}, \ldots, \alpha_{r_{1}-1}, \alpha_{r_{1}+1}, \ldots, \alpha_{r_{2}-1}, \alpha_{r_{2}+1}, \ldots\right): \Omega_{\underline{r}}^{r} \rightarrow$ $\mathbb{G}_{m}^{r-k}$ est lisse de dimension relative $r^{2}$.

Démonstration. Soit $V$ le foncteur qui à tout anneau $A$ associe l'ensemble des $v=\left(v_{1}, v_{2}, \ldots, v_{k}\right)$ vérifiant les conditions de l'énoncé. Se donner un tel $v$ revient à choisir successivement:

- un sous-module $E_{1}$ de $E_{0}=A^{r}$ tel que $E_{0} / E_{1}$ soit localement libre de rang $r_{1}$,

- un homomorphisme $\overline{v_{1}}: E_{0} / E_{1} \rightarrow A^{r}$ partout injectif,

- un sous-module $E_{2}$ de $E_{1}$ tel que $E_{1} / E_{2}$ soit localement libre de rang $r_{2}-r_{1}$,

- un homomorphisme $\overline{v_{2}}: E_{1} / E_{2} \rightarrow A^{r} / \operatorname{Im} \overline{v_{1}}$ partout injectif,

- etc.

Par conséquent, le foncteur $V$ est représentable par un schéma lisse sur $\operatorname{Spec} \mathbb{Z}$ de dimension relative

$$
\begin{aligned}
& \sum_{1 \leq i \leq k}\left[\left(r_{i}-r_{i-1}\right)\left(r-r_{i}\right)+\left(r_{i}-r_{i-1}\right)\left(r-r_{i-1}\right)\right] \\
& =2 r \sum_{1 \leq i \leq k}\left(r_{i}-r_{i-1}\right)-\sum_{1 \leq i \leq k}\left(r_{i}^{2}-r_{i-1}^{2}\right)=r^{2} .
\end{aligned}
$$

Définissons maintenant un morphisme $V \times \mathbb{G}_{m}^{r-k} \rightarrow H^{r}$ dont nous montrerons ensuite qu'il se factorise à travers l'immersion localement fermée $\Omega_{r}^{r} \hookrightarrow H^{r}$ en un isomorphisme $V \times \mathbb{G}_{m}^{r-k} \stackrel{\sim}{\longrightarrow} \Omega_{\underline{r}}^{r}$ au-dessus de $\mathbb{G}_{m}^{r-k}$.

A tout point $\left(v_{1}, \ldots, v_{k} ; \alpha_{1}, \ldots, \alpha_{r_{1}-1}, \alpha_{r_{1}+1}, \ldots, \alpha_{r_{2}-1}, \alpha_{r_{2}+1}, \ldots\right)$ de $V \times$ $\mathbb{G}_{m}^{r-k}$ à valeurs dans un anneau $A$, on associe le point

$$
\left(u_{1}, \ldots, u_{r} ; \alpha_{1}, \ldots, \alpha_{r_{1}-1}, 0, \alpha_{r_{1}+1}, \ldots, \alpha_{r_{2}-1}, 0, \alpha_{r_{2}+1}, \ldots\right)
$$

où $u_{1}, \ldots, u_{r}$ sont définis de la manière suivante: pour $1 \leq i \leq k$ et $r_{i-1}<s \leq r_{i}$, $u_{s}$ est égal à

$$
\left(\prod_{\substack{1 \leq t<s \\ t \neq r_{1}, r_{2}, \ldots, r_{i-1}}} \alpha_{t}^{s-t}\right)^{-1} \operatorname{det}\left(v_{1}\right) \otimes \cdots \otimes \operatorname{det}\left(v_{i-1}\right) \otimes \Lambda^{s-r_{i-1}} v_{i}
$$


où $\operatorname{det}\left(v_{1}\right) \otimes \cdots \otimes \operatorname{det}\left(v_{i-1}\right) \otimes \Lambda^{s-r_{i-1}} v_{i}$ désigne le composé de l'homomorphisme surjectif canonique

$$
\begin{gathered}
\Lambda^{s} A^{r} \longrightarrow \Lambda^{r_{1}}\left(A^{r} / \operatorname{Ker} v_{1}\right) \otimes \Lambda^{r_{2}-r_{1}}\left(\operatorname{Ker} v_{1} / \operatorname{Ker} v_{2}\right) \\
\otimes \cdots \otimes \Lambda^{s-r_{i-1}}\left(\operatorname{Ker} v_{i-1} / \operatorname{Ker} v_{i}\right),
\end{gathered}
$$

du produit tensoriel des isomorphismes induits par $v_{1}, v_{2}, \ldots, v_{i}$

$$
\begin{aligned}
& \Lambda^{r_{1}}\left(A^{r} / \operatorname{Ker} v_{1}\right) \longrightarrow \Lambda^{r_{1}} \operatorname{Im} v_{1}, \\
& \Lambda^{r_{2}-r_{1}}\left(\operatorname{Ker} v_{1} / \operatorname{Ker} v_{2}\right) \longrightarrow \Lambda^{r_{2}-r_{1}} \operatorname{Im} v_{2}, \\
& \ldots \\
& \Lambda^{s-r_{i-1}}\left(\operatorname{Ker} v_{i-1} / \operatorname{Ker} v_{i}\right) \longrightarrow \Lambda^{s-r_{i-1}} \operatorname{Im} v_{i}
\end{aligned}
$$

et de l'homomorphisme injectif canonique

$$
\Lambda^{r_{1}} \operatorname{Im} v_{1} \otimes \Lambda^{r_{2}-r_{1}} \operatorname{Im} v_{2} \otimes \cdots \otimes \Lambda^{s-r_{i-1}} \operatorname{Im} v_{i} \longrightarrow \Lambda^{s} A^{r} .
$$

Afin de montrer que le morphisme $V \times \mathbb{G}_{m}^{r-k} \rightarrow H^{r}$ ainsi défini se factorise à travers l'immersion localement fermée $\Omega_{\underline{r}}^{r} \hookrightarrow H^{r}$ en un isomorphisme $V \times \mathbb{G}_{m}^{r-k} \stackrel{\sim}{\longrightarrow} \Omega_{\underline{r}}^{r}$, il suffit de vérifier que pour tout point de $H^{r}$ à valeurs dans un anneau locăl intègre $A$ tel que le point générique de Spec $A$ s'envoie dans $\Omega_{(r)}^{r}$ et que les conditions " $u_{1}, u_{2}, \ldots, u_{r}$ non nuls, les $\alpha_{s}, s \in \underline{r}$, nuls et les $\alpha_{s}, s \notin \underline{r}$, inversibles" définissent un sous-schéma fermé non vide $\operatorname{Spec} A / J$ de Spec $A$, alors le morphisme $\operatorname{Spec} A / J \rightarrow \Omega_{\underline{r}}^{r} \rightarrow H^{r}$ se factorise de manière unique en $\operatorname{Spec} A / J \rightarrow V \times \mathbb{G}_{m}^{r-k} \rightarrow$ $H^{r}$, et que de plus tout point géométrique de $V \times \mathbb{G}_{m}^{r-k}$ est image d'un tel morphisme $\operatorname{Spec} A / J \rightarrow V \times \mathbb{G}_{m}^{r-k}$.

Soit donc $\left(u_{1}, u_{2}, \ldots, u_{r} ; \alpha_{1}, \ldots, \alpha_{r-1}\right)$ un tel point de $H^{r}$ à valeurs dans $A$. Ainsi $\mathrm{a}-\mathrm{t}-\mathrm{On}$

$$
\Lambda^{s} u_{1}=\left(\prod_{1 \leq t<s} \alpha_{t}^{s-t}\right) u_{s}, \quad 1 \leq s \leq r,
$$

en le point générique $\operatorname{de} \operatorname{Spec} A$. Comme les $u_{s}$ sont non nuls en le point fermé de $\operatorname{Spec} A$, on voit que pour tout $s, 1 \leq s \leq r$, l'idéal de $A$ engendré par les mineurs d'ordre $s$ de $u_{1}$ est principal de générateur l'élément $\prod_{1 \leq t<s} \alpha_{t}^{s-t}$. On en déduit facilement que quitte à composer à droite et à gauche par deux matrices de $\mathrm{GL}_{r}(A), u_{1}$ est de la forme

$$
u_{1}=\left(\begin{array}{ccccc}
1 & & & & \\
& \alpha_{1} & & & \\
& & \alpha_{1} \alpha_{2} & & 0 \\
& 0 & & \ddots & \\
& & & & \alpha_{1} \alpha_{2} \cdots \alpha_{r-1}
\end{array}\right) .
$$

Ce qu'on voulait en résulte immédiatement.

La proposition 1 a la conséquence immédiate suivante:

Corollaire 2. Le morphisme $\left(\alpha_{1}, \alpha_{2}, \ldots, \alpha_{r}\right): \Omega^{r} \rightarrow \mathbb{A}^{r-1}$ est lisse de dimension relative $r^{2}$. 
Le quotient de $\Omega^{r}$ par l'action libre du tore $\mathbb{G}_{m}^{r}$ est l'adhérence schématique de l'orbite du groupe $\mathrm{PGL}_{r} \times \mathrm{PGL}_{r}$ agissant sur $\prod_{0 \leq s \leq r}^{m} \mathbb{P}\left(\Lambda^{s} \mathbb{A}^{r} \otimes \Lambda^{r-s} \mathbb{A}^{r}\right)$ engendrée par le point associé à l'immersion diagonale de $\mathbb{A}^{r}$ dans $\mathbb{A}^{r} \oplus \mathbb{A}^{r}$; il est projectif, contient $\left(\mathrm{PGL}_{r} \times \mathrm{PGL}_{r}\right) / \mathrm{PGL}_{r}$ comme ouvert dense et a pour bord un diviseur à croisements normaux. C'est un cas particulier des compactifications de De Concini et Procesi.

b) Le champ des pré-chtoucas itérés. On fixe $X$ une courbe projective lisse géométriquement connexe au-dessus d'un corps fini $\mathbb{F}_{q}$.

Etant donnés $S$ un schéma sur $\mathbb{F}_{q}, \mathcal{E}$ un $\mathcal{O}_{X \times S}$-Module localement libre de rang $r$ et $\infty, 0: S \rightarrow X$ deux morphismes, on appelle modification (à droite) de $\mathcal{E}$ de pôle $\infty$ et de zéro 0 tout diagramme

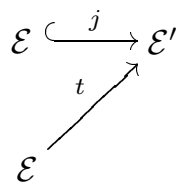

où $\mathcal{E}^{\prime}, \mathcal{E}^{\prime \prime}$ sont des $\mathcal{O}_{X \times S^{-}}$Modules localement libres de rang $r$, et $j, t$ sont des homomorphismes $\mathcal{O}_{X \times S^{-}}$-linéaires injectifs dont les conoyaux sont supportés par les graphes de $\infty, 0$ respectivement et sont localement libres de rang 1 sur $\mathcal{O}_{S}$.

D'autre part, dans cette même situation, on notera ${ }^{\tau} \mathcal{E}$ l'image réciproque de $\mathcal{E}$ par le morphisme $\operatorname{Id}_{X} \times$ Frob $_{S}$.

On rappelle qu'un chtouca (à droite) de rang $r$ sur $S$ consiste en la donnée

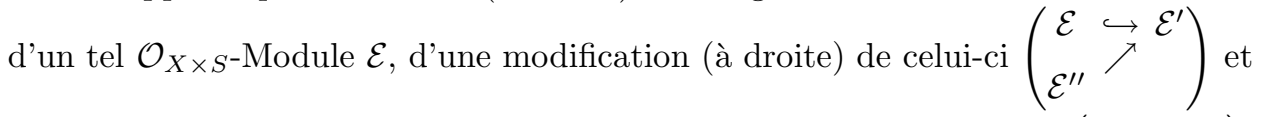
d'un isomorphisme ${ }^{\tau} \mathcal{E} \stackrel{\sim}{\rightarrow} \mathcal{E}^{\prime \prime}$, c'est-à-dire en résumé d'un diagramme $\left(\begin{array}{cc}\mathcal{E} & \hookrightarrow \\ \tau \mathcal{E} & \mathcal{E}^{\prime}\end{array}\right)$ soumis à certaines conditions.

Maintenant, posons:

Définition 3. Pour $r \geq 1$ un entier et $S$ un schéma sur $\mathbb{F}_{q}$, on appelle pré-chtouca itéré (à droite) de rang $r$ sur $S$ la donnée de

- un $\mathcal{O}_{X \times S}$-Module $\mathcal{E}$ sur $X \times S$ localement libre de rang $r$,

- une modification (à droite) $\left(\begin{array}{lll}\mathcal{E} & \mathcal{E}^{\prime} \\ \mathcal{E}^{\prime \prime} & \nearrow\end{array}\right)$ de $\mathcal{E}$,

- des $\mathcal{O}_{S}$-Modules inversibles $\mathcal{L}_{1}, \mathcal{L}_{2}, \ldots, \mathcal{L}_{r-1}$ sur $S$, munis de sections globales $\ell_{1}, \ell_{2}, \ldots, \ell_{r-1}$, libres

- pour tout $s, 1 \leq s \leq r$, un homomorphisme de $\mathcal{O}_{X \times S}$-Modules localement

$$
\tau\left(\Lambda^{s} \mathcal{E} \otimes \bigotimes_{1 \leq t<s} \mathcal{L}_{t}^{\otimes(s-t)}\right) \stackrel{u_{s}}{\longrightarrow} \Lambda^{s} \mathcal{E}^{\prime \prime} \otimes \bigotimes_{1 \leq t<s} \mathcal{L}_{t}^{\otimes(s-t)}
$$

qui peut aussi se voir comme

$$
\Lambda^{s}\left({ }^{\tau} \mathcal{E}\right) \otimes \bigotimes_{1 \leq t<s} \mathcal{L}_{t}^{\otimes(q-1)(s-t)} \stackrel{u_{s}}{\longrightarrow} \Lambda^{s} \mathcal{E}^{\prime \prime}
$$

ces données étant soumises aux deux conditions suivantes: 
(i) Pour un (et donc pour tout) choix de trivialisations de $\mathcal{L}_{1}, \mathcal{L}_{2}, \ldots, \mathcal{L}_{r-1}$ localement sur $S$ et de $\mathcal{E}, \mathcal{E}^{\prime \prime}$ localement sur $X \times S$, la famille $\left(u_{1}, u_{2}, \ldots, u_{r}\right.$; $\left.\ell_{1}^{q-1}, \ell_{2}^{q-1}, \ldots, \ell_{r-1}^{q-1}\right)$ prend ses valeurs dans le sous-schéma localement fermé $\Omega^{r}$ de $H^{r}$.

(ii) $S$ i on identifie $\mathcal{E}$ et sa modification $\mathcal{E}^{\prime \prime}$ sur l'ouvert de $X \times S$ complémentaire des graphes du pôle et du zéro de cette modification, alors aucun des homomorphismes induits

$$
\tau\left(\Lambda^{s} \mathcal{E} \otimes \bigotimes_{1 \leq t<s} \mathcal{L}_{t}^{\otimes(s-t)}\right) \stackrel{u_{s}}{\longrightarrow} \Lambda^{s} \mathcal{E} \otimes \bigotimes_{1 \leq t<s} \mathcal{L}_{t}^{\otimes(s-t)}
$$

n'est nilpotent au-dessus d'aucun des points géométriques de S.

Remarquons tout de suite:

Lemme 4. La condition (ii) dans la définition 3 est équivalente à ce que génériquement au-dessus de tout point géométrique de $S$ chaque homomorphisme

$$
\tau\left(\Lambda^{s} \mathcal{E} \otimes \bigotimes_{1 \leq t<s} \mathcal{L}_{t}^{\otimes(s-t)}\right) \stackrel{u_{s}}{\longrightarrow} \Lambda^{s} \mathcal{E} \otimes \bigotimes_{1 \leq t<s} \mathcal{L}_{t}^{\otimes(s-t)}
$$

ait son noyau et le transformé par $\tau$ de son image en somme directe (autrement dit que toutes ses puissances aient même rang que lui).

Démonstration. On peut supposer que $S$ est le spectre d'un corps algébriquement clos. Il est évident que la nouvelle condition ci-dessus entraîne la condition (ii).

Réciproquement, supposons satisfaite ladite condition (ii). Notons $r_{1}, r_{2}, \ldots$ ceux des $t, 1 \leq t<r$, tels que $\ell_{t}=0$. D'après la proposition $1 \mathrm{du}$ premier paragraphe, il suffit de vérifier que toutes les puissances de $u_{1}, u_{r_{1}+1}, u_{r_{2}+1}, \ldots$ sont de rangs $r_{1}, r_{2}, r_{3}, \ldots$ et pour cela que $u_{r_{1}}, u_{r_{2}}, u_{r_{3}}, \ldots$ ne sont pas nilpotents, ce qui est vrai par hypothèse.

Pour tout entier $r \geq 1$, on notera $\mathcal{C}^{r}$ la catégorie fibrée qui à tout schéma $S$ sur $\mathbb{F}_{q}$ associe le groupoïde $\mathcal{C}^{r}(S)$ des pré-chtoucas itérés de rang $r$ sur $S$. Il est immédiat que c'est un champ pour la topologie f.p.q.c.

Proposition 5. Pour tout entier $r \geq 1$, le champ $\mathcal{C}^{r}$ des pré-chtoucas itérés de rang $r$ est algébrique (au sens d'Artin) et localement de type fini.

Démonstration. La catégorie fibrée qui à tout schéma $S$ sur $\mathbb{F}_{q}$ associe le groupoïde des familles de fibrés inversibles $\mathcal{L}_{1}, \mathcal{L}_{2}, \ldots, \mathcal{L}_{r-1}$ sur $S$, munis de sections globales $\ell_{1}, \ell_{2}, \ldots, \ell_{r-1}$, n'est autre que le champ algébrique $\left(\mathbb{A}^{1} / \mathbb{G}_{m}\right)^{r-1}$.

D'autre part, on sait que la catégorie fibrée qui à tout schéma $S$ sur $\mathbb{F}_{q}$ associe le groupoïde des $\mathcal{O}_{X \times S^{-}}$-Modules $\mathcal{E}$ localement libres de rang $r$ sur $X \times S$ est un champ algébrique localement de type fini.

Puis le choix d'une modification $\left(\begin{array}{lll}\mathcal{E} & \hookrightarrow & \mathcal{E}^{\prime} \\ \mathcal{E}^{\prime \prime} & \nearrow\end{array}\right)$ est représentable par un morphisme projectif.

Maintenant, la condition (i) de la définition 1 est localement fermée sur chaque $X \times S$ donc aussi sur chaque $S$ (voir [4], lemme $3 \mathrm{du}$ paragraphe I.2).

Et la condition (ii) est évidemment ouverte sur chaque base $S$. 
c) Description des différentes strates. Le champ des chtoucas itérés. Fixons une partition $\underline{r}=\left(r_{1}, \ldots, r_{k}\right)$ de $r$ avec donc $0<r_{1}<\cdots<r_{k}=r$. Pour tout $s \in \underline{r}$, on notera $s^{-}$son prédécesseur dans $\underline{r}^{-}=\left(r_{0}=0, r_{1}, \ldots, r_{k-1}\right)$ et pour tout $s \in \underline{r}^{-}$, on notera $s^{+}$son successeur dans $\underline{r}$.

Notons $\mathcal{C}_{\underline{r}}^{r}$ le sous-champ algébrique localement fermé de $\mathcal{C}^{r}$ défini par les conditions suivantes relatives aux sections $\ell_{1}, \ell_{2}, \ldots, \ell_{r-1}$ de $\mathcal{L}_{1}, \mathcal{L}_{2}, \ldots, \mathcal{L}_{r-1}$ :

- les $\ell_{s}, s \in \underline{r}$, sont nulles,

- les $\ell_{s}, s \notin \underline{r}$, sont partout inversibles.

$\mathcal{C}_{\underline{r}}^{r}$ pourra être appelé le champ des pré-chtoucas itérés de type $\underline{r}$.

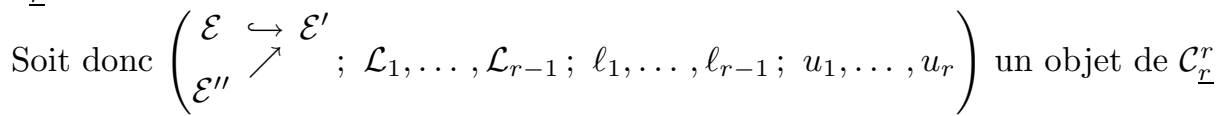
au-dessus d'un schéma $S$ sur $\mathbb{F}_{q}$. Pour tout $s \notin \underline{r}$, le fibré $\mathcal{L}_{s}$ sur $S$ muni de la section inversible $\ell_{s}$ peut être identifié au fibré trivial $\mathcal{O}_{S}$ muni de la section 1 . Ceci étant dit et d'après la proposition 1 du premier paragraphe, la donnée de la famille d'homomorphismes $u_{1}, u_{2}, \ldots, u_{r}$ est équivalente à la donnée de

- une filtration décroissante ${ }^{\tau} \mathcal{E}=\overline{\mathcal{E}}_{0} \supseteq \cdots \nsupseteq \overline{\mathcal{E}}_{s} \supseteq \cdots \nsupseteq \overline{\mathcal{E}}_{r}=0$ de ${ }^{\tau} \mathcal{E}$ par des $\mathcal{O}_{X \times S^{-}}$Modules localement libres, telle que les quotients successifs $\overline{\mathcal{E}}_{s^{-}} / \overline{\mathcal{E}}_{s}, s \in \underline{r}$, soient localement libres de rangs $s-s^{-}$,

- une filtration croissante $0=\mathcal{E}_{0}^{\prime \prime} \varsubsetneqq \cdots \varsubsetneqq \mathcal{E}_{s}^{\prime \prime} \varsubsetneqq \cdots \varsubsetneqq \mathcal{E}_{r}^{\prime \prime}=\mathcal{E}^{\prime \prime}$ de $\mathcal{E}^{\prime \prime}$ par des $\mathcal{O}_{X \times S^{-}}$Modules localement libres, telle que les quotients successifs $\mathcal{E}_{s}^{\prime \prime} / \mathcal{E}_{s^{-}}^{\prime \prime}, s \in \underline{r}$, soient localement libres de rangs $s-s^{-}$,

- une famille d'isomorphismes, $s \in \underline{r}$,

$$
\overline{\mathcal{E}}_{s^{-}} / \overline{\mathcal{E}}_{s} \otimes \tau\left(\bigotimes_{\substack{t \in \underline{r} \\ t<s}} \mathcal{L}_{t}\right) \stackrel{\sim}{\longrightarrow} \mathcal{E}_{s}^{\prime \prime} / \mathcal{E}_{s^{-}}^{\prime \prime} \otimes\left(\bigotimes_{\substack{t \in \underline{r} \\ t<\bar{s}}} \mathcal{L}_{t}\right)
$$

Lemme 6. Avec les notations ci-dessus, les trois conditions suivantes définissent un sous-champ ouvert $\overline{\mathrm{Cht}_{\underline{r}}^{r}}$ de $\mathcal{C}_{\underline{r}}^{r}$, qu'on appellera le champ des chtoucas itérés de type $\underline{r}$ :

(i) En notant $\mathcal{E}_{s}^{\prime}=\mathcal{E}_{s}^{\prime \prime}$ si $s \in \underline{r}^{-}$et $\mathcal{E}_{r}^{\prime}=\mathcal{E}^{\prime}$, tous les quotients $\mathcal{E}^{\prime} / \mathcal{E}_{s}^{\prime}$ sont localement libres sur $\mathcal{O}_{X \times S}$.

(ii) Pour tout $s \in \underline{r}$, l'homomorphisme $\mathcal{E}_{s}^{\prime} \longrightarrow \mathcal{E}^{\prime} / \mathcal{E}$ est surjectif, si bien que son noyau $\mathcal{E}_{s}$ est localement libre sur $\mathcal{O}_{X \times S}$, tout comme $\mathcal{E}_{0}=0$.

(iii) Pour tout $s \in \underline{r}$, on a $\overline{\mathcal{E}}_{s^{-}}+{ }^{\tau} \mathcal{E}_{s}={ }^{\tau} \mathcal{E}$.

De plus, il existe un unique sous-champ ouvert $\overline{\mathrm{Cht}^{r}}$ de $\mathcal{C}^{r}$ dont la trace dans chaque strate $\mathcal{C}_{\underline{r}}^{r}$ soit égale à $\overline{\mathrm{Cht}_{\underline{r}}^{r}}$.

On appellera $\overline{\mathrm{Cht}^{r}}$ le champ des chtoucas itérés de rang $r$.

Démonstration. La première assertion est évidente.

La seconde résulte de la première et de ce que toute strate $\mathcal{C}_{\underline{r}}^{r}$ de $\mathcal{C}^{r}$ est un ouvert dans le fermé réunion des $\mathcal{C}_{\underline{r}^{\prime}}^{r}, \underline{r}^{\prime} \supseteq \underline{r}$. On utilise le fait qu'au-dessus de ce fermé les $\overline{\mathcal{E}}_{s}$ et $\mathcal{E}_{s}^{\prime \prime}, s \in \underline{r}$, se recollent en des $\mathcal{O}_{X \times S}$-Modules localement libres. 
Supposons maintenant que notre pré-chtouca itéré

$$
\left(\begin{array}{l}
\mathcal{E} \\
\mathcal{E}^{\prime \prime}
\end{array} \stackrel{\mathcal{E}}{\prime}^{\prime} ; \mathcal{L}_{1}, \ldots, \mathcal{L}_{r-1} ; \ell_{1}, \ldots, \ell_{r-1} ; u_{1}, \ldots, u_{r}\right)
$$

est un chtouca itéré de type $\underline{r}$ et poursuivons la discussion, en usant des notations déjà introduites.

Notons $\mathcal{A}_{r_{1}}=\mathcal{E}_{r_{1}} \subseteq \mathcal{E}$ et $\mathcal{A}_{r_{1}}^{\prime}=\mathcal{E}_{r_{1}}^{\prime}=\mathcal{E}_{r_{1}}^{\prime \prime}$. Alors le composé ${ }^{\tau} \mathcal{A}_{r_{1}} \hookrightarrow{ }^{\tau} \mathcal{E} \rightarrow$ $\tau \mathcal{E} / \overline{\mathcal{E}}_{r_{1}} \stackrel{\sim}{\longrightarrow} \mathcal{E}_{r_{1}}^{\prime \prime}=\mathcal{A}_{r_{1}}^{\prime}$ s'intègre dans un diagramme $\left(\begin{array}{ccc}\mathcal{A}_{r_{1}} & \hookrightarrow & \mathcal{A}_{r_{1}}^{\prime} \\ & \nearrow & \end{array}\right)=\widetilde{\mathcal{A}}_{r_{1}}$ qui définit un chtouca à droite de rang $r_{1}$ sur $S$. De plus, on a un isomorphisme canonique $\mathcal{A}_{r_{1}}^{\prime} / \mathcal{A}_{r_{1}} \stackrel{\sim}{\longrightarrow} \mathcal{E}^{\prime} / \mathcal{E}$ d'où il ressort que ce chtouca a même pôle que la modification $\left(\begin{array}{lll}\mathcal{E} & \hookrightarrow & \mathcal{E}^{\prime} \\ & \nearrow & \\ \mathcal{E}^{\prime \prime} & & \end{array}\right)$.

Puis, pour $s \in \underline{r}, s>r_{1}=0^{+}$, notons $\mathcal{A}_{s}^{\prime}=\overline{\mathcal{E}}_{s^{-}} \cap{ }^{\tau} \mathcal{E}_{s}$ le noyau de l'homomorphisme surjectif $\overline{\mathcal{E}}_{s^{-}} \oplus{ }^{\tau} \mathcal{E}_{s} \rightarrow{ }^{\tau} \mathcal{E}$. Et notons $\mathcal{A}_{s}=\mathcal{E}_{s} / \mathcal{E}_{s^{-}} \cong \mathcal{E}_{s}^{\prime} / \mathcal{E}_{s^{-}}^{\prime}$. On dispose des deux composés

$$
\mathcal{A}_{s}^{\prime} \hookrightarrow{ }^{\tau} \mathcal{E}_{s} \rightarrow{ }^{\tau} \mathcal{E}_{s} /{ }^{\tau} \mathcal{E}_{s^{-}}={ }^{\tau} \mathcal{A}_{s}
$$

et

$$
\begin{gathered}
\mathcal{A}_{s}^{\prime} \otimes^{\tau}\left(\bigotimes_{\substack{t \in \underline{r} \\
t<\bar{s}}} \mathcal{L}_{t}\right) \hookrightarrow \overline{\mathcal{E}}_{s^{-}} \otimes^{\tau}\left(\bigotimes_{\substack{t \in \underline{r} \\
t<s}} \mathcal{L}_{t}\right) \longrightarrow \overline{\mathcal{E}}_{s^{-}} / \overline{\mathcal{E}}_{s} \otimes^{\tau}\left(\bigotimes_{\substack{t \in \underline{r} \\
t<s}} \mathcal{L}_{t}\right) \\
\longrightarrow \mathcal{E}_{s}^{\prime \prime} / \mathcal{E}_{s^{-}}^{\prime \prime} \otimes\left(\bigotimes_{\substack{t \in \underline{r} \\
t<s}} \mathcal{L}_{t}\right) \hookrightarrow \mathcal{A}_{s} \otimes\left(\bigotimes_{\substack{t \in \underline{r} \\
t<s}} \mathcal{L}_{t}\right) .
\end{gathered}
$$

Ils s'intègrent dans un diagramme

$$
\left(\begin{array}{ccc} 
& \mathcal{A}_{s} \otimes\left(\otimes_{\substack{t \in r \\
t<s}} \mathcal{L}_{t}\right) \\
\mathcal{A}_{s}^{\prime} \otimes \tau\left(\otimes_{\substack{t \in r \\
t<s}} \mathcal{L}_{t}\right) & \nearrow & \tau^{\tau} \mathcal{A}_{s} \otimes \tau\left(\otimes_{\substack{t \in r \\
t<s}} \mathcal{L}_{t}\right)
\end{array}\right)=\widetilde{\mathcal{A}}_{s}
$$

qui définit un chtouca à gauche de rang $s-s_{-}$au-dessus de $S$.

Lorsque $s=r$ est le plus grand élément de $\underline{r}$, on a un isomorphisme canonique

$$
\mathcal{A}_{r} \otimes\left(\bigotimes_{\substack{t \in \underline{r} \\ t<r}} \mathcal{L}_{t}\right) / \mathcal{A}_{r}^{\prime} \otimes \tau\left(\bigotimes_{\substack{t \in \underline{r} \\ t<r}} \mathcal{L}_{t}\right) \stackrel{\sim}{\longrightarrow} \mathcal{E}^{\prime} / \mathcal{E}^{\prime \prime} \otimes\left(\bigotimes_{\substack{t \in \underline{r} \\ t<r}} \mathcal{L}_{t}\right)
$$

d'où il ressort que le zéro de $\widetilde{\mathcal{A}}_{r}$ se confond avec celui de la modification $\left(\begin{array}{cc}\mathcal{E} & \hookrightarrow \\ & \nearrow \\ \mathcal{E}^{\prime \prime} & \nearrow\end{array}\right)$. 
D'autre part, pour $s \in \underline{r} \cap \underline{r}^{-}$c'est-⿳亠े⿵-dire $0^{+}=r_{1} \leq s \leq r_{k-1}=r^{-}$, on a des isomorphismes canoniques

$$
\begin{aligned}
& \tau \mathcal{E} /\left(\overline{\mathcal{E}}_{s} \oplus{ }^{\tau} \mathcal{E}_{s}\right) \cong\left(\overline{\mathcal{E}}_{s} \oplus{ }^{\tau} \mathcal{E}_{s^{+}}\right) /\left(\overline{\mathcal{E}}_{s} \cap{ }^{\tau} \mathcal{E}_{s^{+}}\right) /\left(\overline{\mathcal{E}}_{s} \oplus{ }^{\tau} \mathcal{E}_{s}\right) \\
& \cong\left({ }^{\tau} \mathcal{E}_{s^{+}} /{ }^{\tau} \mathcal{E}_{s}\right) /\left(\overline{\mathcal{E}}_{s} \cap{ }^{\tau} \mathcal{E}_{s^{+}}\right)={ }^{\tau} \mathcal{A}_{s^{+}} / \mathcal{A}_{s^{+}}^{\prime} \text { et } \\
& \tau \mathcal{E} /\left(\overline{\mathcal{E}}_{s} \oplus^{\tau} \mathcal{E}_{s}\right) \cong\left(\overline{\mathcal{E}}_{s^{-}} \oplus^{\tau} \mathcal{E}_{s}\right) /\left(\overline{\mathcal{E}}_{s^{-}} \cap{ }^{\tau} \mathcal{E}_{s}\right) /\left(\overline{\mathcal{E}}_{s} \oplus^{\tau} \mathcal{E}_{s}\right) \\
& \cong\left(\overline{\mathcal{E}}_{s^{-}} / \overline{\mathcal{E}}_{s}\right) /\left(\overline{\mathcal{E}}_{s^{-}} \cap{ }^{\tau} \mathcal{E}_{s}\right) \\
& \cong\left\{\begin{array}{l}
\mathcal{A}_{r_{1}}^{\prime} /{ }^{\tau} \mathcal{A}_{r_{1}} \text { si } s=r_{1}, \\
\mathcal{A}_{s} \otimes{ }^{1-\tau}\left(\bigotimes_{t \in \underline{r}} \mathcal{L}_{t}\right) / \mathcal{A}_{s}^{\prime} \text { si } s>r_{1},
\end{array}\right.
\end{aligned}
$$

si bien que le zéro de $\widetilde{\mathcal{A}}_{s}$ se confond avec le pôle de $\widetilde{\mathcal{A}}_{s^{+}}$.

Proposition 7. Soit $\underline{r}=\left(r_{1}, \ldots, r_{k}\right)$ une partition de l'entier $r$, avec donc $0<$ $r_{1}<\cdots<r_{k-1}<r_{k}=r$.

Soit $\mathrm{Cht}^{\underline{\underline{r}}}$ la catégorie fibrée qui à tout schéma $S$ sur $\mathbb{F}_{q}$ associe le groupoüde des familles constituées de

- des $\mathcal{O}_{S}$-Modules inversibles sur $S$ au nombre de $k-1$ que l'on note $\mathcal{L}_{s}, s \in \underline{r}$, $s<r_{k}=r$,

- un chtouca à droite $\widetilde{\mathcal{A}}_{r_{1}}=\left(\begin{array}{ccc}\mathcal{A}_{r_{1}} & \hookrightarrow & \mathcal{A}_{r_{1}}^{\prime} \\ & \nearrow & \end{array}\right)$ de rang $r_{1}$ au-dessus de $S$ et $k-1$ chtoucas à gauche de rangs respectifs $s-s_{-}, s \in \underline{r}, s>r_{1}$, qu'on écrit sous la forme

$$
\widetilde{\mathcal{A}}_{s}=\left(\begin{array}{ccc} 
& & \mathcal{A}_{s} \otimes\left(\bigotimes_{\substack{t \in \underline{r} \\
t<s}} \mathcal{L}_{t}\right) \\
\mathcal{A}_{s}^{\prime} \otimes \tau\left(\bigotimes_{\substack{t \in \underline{r} \\
t<s}} \mathcal{L}_{t}\right) & \hookrightarrow & \tau_{\mathcal{A}_{s}} \otimes \\
\tau & & \\
& & \left.\bigotimes_{\substack{t \in \underline{r} \\
t<s}} \mathcal{L}_{t}\right)
\end{array}\right),
$$

- des isomorphismes de $\mathcal{O}_{X \times S}-$ Modules

$$
\begin{gathered}
\mathcal{A}_{r_{1}}^{\prime} /{ }^{\tau} \mathcal{A}_{r_{1}} \stackrel{\sim}{\longrightarrow}{ }^{\tau} \mathcal{A}_{r_{2}} / \mathcal{A}_{r_{2}}^{\prime} \quad \text { et } \\
\mathcal{A}_{s} \otimes{ }^{1-\tau}\left(\bigotimes_{\substack{t \in \underline{r} \\
t<s}} \mathcal{L}_{t}\right) / \mathcal{A}_{s}^{\prime} \stackrel{\sim}{\longrightarrow} \mathcal{A}_{s^{+}} / \mathcal{A}_{s^{+}}^{\prime}, \quad s \in \underline{r}, r_{1}<s<r .
\end{gathered}
$$

Alors:

(i) La catégorie fibrée $\mathrm{Cht}^{\underline{r}}$ est un champ algébrique au sens de Deligne-Mumford, séparé et muni d'un morphisme naturel

$$
\mathrm{Cht}^{\underline{r}} \longrightarrow X \times X \times X^{k-1}
$$

qui est localement de type fini et lisse de dimension relative $2 r-2 k$.

(ii) La discussion qui précède l'énoncé de la présente proposition définit un morphisme naturel

$$
\overline{\mathrm{Cht}_{\underline{r}}^{r}} \longrightarrow \mathrm{Cht}^{\underline{r}}
$$

qui est fini, surjectif et radiciel.

(iii) Il existe une constante $\mu \geq 0$ telle que le morphisme

$$
\overline{\mathrm{Cht}_{\underline{r}}^{r}} \longrightarrow \mathrm{Cht}^{\underline{r}}
$$


devienne une gerbe (dont le groupe de structure est plat, fini et radiciel) au-dessus de l'ouvert de Cht ${ }^{\underline{\underline{T}}}$ défini par les conditions

$$
\mu^{-}\left(\widetilde{\mathcal{A}}_{s}\right) \geq \mu+\mu^{+}\left(\widetilde{\mathcal{A}}_{s^{+}}\right), s \in \underline{r}, s<r .
$$

$($ Pour $\mathcal{E}$ un fibré non nul sur une courbe projective lisse, on note $\mu(\mathcal{E})=$ $\operatorname{deg}(\mathcal{E}) / \operatorname{rg}(\mathcal{E})$ et $\mu^{+}(\mathcal{E})$ le maximum [resp. $\mu^{-}(\mathcal{E})$ le minimum] des $\mu(\mathcal{F})$ quand $\mathcal{F}$ décrit l'ensemble des fibrés non nuls qui sont sous-objets [resp. objets quotients] de $\mathcal{E}$.)

En particulier, au-dessus de cet ouvert, $\overline{\mathrm{Cht}_{\underline{r}}^{r}}$ est lisse sur $X \times X \times X^{k-1}$ de dimension relative $2 r-2 k$.

Remarque. C'est en pensant aux énoncés (ii) et (iii) qu'on a baptisé $\overline{\mathrm{Cht}_{\underline{r}}^{r}}$ champ des chtoucas itérés de type $\underline{r}$.

Démonstration de la proposition \%. (i) On sait que le champ $\mathrm{Cht}^{r_{1}}$ des chtoucas à droite de rang $r_{1}$ et les champs ${ }^{r_{2}-r_{1}}$ Cht $, \ldots,{ }^{r_{k}-r_{k-1}}$ Cht des chtoucas à gauche de rangs $r_{2}-r_{1}, \ldots, r_{k}-r_{k-1}$ sont algébriques au sens de Deligne-Mumford, séparés, localement de type fini et lisses de dimensions relatives

$$
2 r_{1}-2,2\left(r_{2}-r_{1}\right)-2, \ldots, 2\left(r_{k}-r_{k-1}\right)-2
$$

au-dessus de $X \times X$.

Par conséquent, le champ

$$
\mathrm{Cht}^{r_{1}} \times_{X}{ }^{r_{2}-r_{1}} \mathrm{Cht} \times_{X} \cdots \times_{X}{ }^{r_{k}-r_{k-1}} \mathrm{Cht}
$$

est lui-même algébrique au sens de Deligne-Mumford, séparé, localement de type fini et lisse de dimension relative $2 r-2 k$ au-dessus de $X \times X \times X^{k-1}$.

On en déduit le résultat annoncé.

(ii) On considère un objet de $\mathrm{Cht}^{\underline{\underline{r}}}$ au-dessus d'un schéma $S$ sur $\mathbb{F}_{q}$, comme précisé dans l'énoncé de la proposition.

On cherche à décrire la fibre au-dessus de cet objet du morphisme $\overline{\mathrm{Cht}_{r}^{r}} \rightarrow \mathrm{Cht}^{\underline{r}}$. Et d'après la discussion qui précède l'énoncé du lemme 6 , un objet de $\overline{\mathrm{Cht}_{\underline{r}}^{r}}$ est constitué d'une modification $\left(\begin{array}{ccc}\mathcal{E} & \hookrightarrow & \mathcal{E}^{\prime} \\ & \nearrow & \\ \mathcal{E}^{\prime \prime} & & \end{array}\right)$, d'une famille de fibrés inversibles $\mathcal{L}_{s}, s \in \underline{r}, s<r$, de filtrations ${ }^{\tau} \mathcal{E}=\overline{\mathcal{E}}_{0} \supseteq \cdots \nsupseteq \overline{\mathcal{E}}_{s} \supseteq \cdots \nsupseteq \overline{\mathcal{E}}_{r}=0,0=$ $\mathcal{E}_{0}^{\prime \prime} \varsubsetneqq \cdots \varsubsetneqq \mathcal{E}_{s}^{\prime \prime} \varsubsetneqq \cdots \varsubsetneqq \mathcal{E}_{r}^{\prime \prime}=\mathcal{E}^{\prime \prime}, 0=\mathcal{E}_{0}^{\prime} \varsubsetneqq \cdots \varsubsetneqq \mathcal{E}_{s}^{\prime} \varsubsetneqq \cdots \varsubsetneqq \mathcal{E}_{r}^{\prime}=\mathcal{E}^{\prime}$ et $0=\mathcal{E}_{0} \varsubsetneqq \cdots \varsubsetneqq \mathcal{E}_{s} \varsubsetneqq \cdots \varsubsetneqq \mathcal{E}_{r}=\mathcal{E}$ telles que $\mathcal{E}_{s}^{\prime} / \mathcal{E}_{s}=\mathcal{E}^{\prime} / \mathcal{E}, \forall s \in \underline{r}$, et $\mathcal{E}_{s}^{\prime}=\mathcal{E}_{s}^{\prime \prime}$, $\forall s \in \underline{r}^{-}$, et d'une famille d'isomorphismes

$$
\overline{\mathcal{E}}_{s^{-}} / \overline{\mathcal{E}}_{s} \otimes \tau\left(\bigotimes_{\substack{t \in \underline{r} \\ t<s}} \mathcal{L}_{t}\right) \stackrel{\sim}{\longrightarrow} \mathcal{E}_{s}^{\prime \prime} / \mathcal{E}_{s^{-}}^{\prime \prime} \otimes\left(\bigotimes_{\substack{t \in \underline{r} \\ t<s}} \mathcal{L}_{t}\right)
$$

Tout d'abord, il existe une unique façon de reconstituer ${ }^{\tau} \mathcal{E}$ muni de ses deux filtrations ${ }^{\tau} \mathcal{E}=\overline{\mathcal{E}}_{0} \supseteq \cdots \nsupseteq \overline{\mathcal{E}}_{s} \supseteq \cdots \nsupseteq \overline{\mathcal{E}}_{r}=0$ et $0={ }^{\tau} \mathcal{E}_{0} \varsubsetneqq \cdots \varsubsetneqq{ }^{\tau} \mathcal{E}_{s} \varsubsetneqq \cdots \varsubsetneqq{ }^{\tau} \mathcal{E}_{r}=$ ${ }^{\tau} \mathcal{E}$ à partir de l'objet considéré de $\mathrm{Cht}^{\underline{r}}$. Pour le voir, commençons par écrire la somme directe

$$
\mathcal{A}=\mathcal{A}_{r_{1}}^{\prime} \oplus \bigoplus_{\substack{s \in \underline{r} \\ r_{1}<s<r}}\left(\tau \mathcal{A}_{s} \oplus \mathcal{A}_{s} \otimes^{1-\tau}\left(\bigotimes_{\substack{t \in \underline{r} \\ t<s}} \mathcal{L}_{t}\right)\right) \oplus^{\tau} \mathcal{A}_{r}
$$


Il y a deux plongements naturels $a$ et $a^{\prime}$ de la somme directe $\bigoplus_{\substack{s \in \underline{r} \\ r_{1}<s<r}} \mathcal{A}_{s}^{\prime}$ dans $\mathcal{A}$. Ils s'obtiennent en faisant la somme directe de tous les homomorphismes de pôles ou bien de zéros dans les diagrammes $\widetilde{\mathcal{A}}_{s}, s \in \underline{r}, r_{1}<s<r$. D'autre part, il y a deux homomorphismes surjectifs naturels $\bar{a}$ et $\bar{a}^{\prime}$ de $\mathcal{A}$ dans la somme directe de quotients

$$
\mathcal{A}_{r_{1}}^{\prime} /{ }^{\tau} \mathcal{A}_{r_{1}} \oplus \bigoplus_{\substack{s \in \underline{r} \\ r_{1}<s<r}}\left(\mathcal{A}_{s} \otimes^{1-\tau}\left(\bigotimes_{\substack{t \in \underline{r} \\ t<s}} \mathcal{L}_{t}\right)\right) / \mathcal{A}_{s}^{\prime} \cong \bigoplus_{\substack{s \in \underline{r} \\ r_{1}<s}} \mathcal{A}_{s} / \mathcal{A}_{s}^{\prime}
$$

Alors on a ${ }^{\tau} \mathcal{E}=\operatorname{Ker}\left(\bar{a}^{\prime}-\bar{a}\right) / \operatorname{Im}\left(a^{\prime}-a\right)$.

Puis on cherche à construire $\mathcal{E}$ muni de la filtration $0=\mathcal{E}_{0} \varsubsetneqq \cdots \varsubsetneqq \mathcal{E}_{s} \varsubsetneqq \cdots \varsubsetneqq$ $\mathcal{E}_{r}=\mathcal{E}$, connaissant déjà ${ }^{\tau} \mathcal{E}$ et les quotients successifs $\mathcal{E}_{s} / \mathcal{E}_{s^{-}}=\mathcal{A}_{s}, s \in \underline{r}$.

En notant $\operatorname{Vec}_{X}^{r_{1}}, \operatorname{Vec}_{X}^{r_{2}-r_{1}}, \ldots, \operatorname{Vec}_{X}^{r_{k}-r_{k-1}}$ les champs classifiant les fibrés localement libres de rangs $r_{1}, r_{2}-r_{1}, \ldots, r_{k}-r_{k-1}$ sur $X$ et $\operatorname{Vec} \frac{r}{X}$ le champ classifiant les fibrés de rang $r$ sur $X$ qui sont munis d'une filtration dont les quotients successifs sont des fibrés localement libres de rangs $r_{1}, r_{2}-r_{1}, \ldots, r_{k}-r_{k-1}$, le problème de construire cet $\mathcal{E}$ est représenté par une fibre du morphisme

$$
\operatorname{Vec}_{X}^{\frac{r}{X}} \longrightarrow\left(\operatorname{Vec}_{X}^{r_{1}} \times \cdots \times \operatorname{Vec}_{X}^{r_{k}-r_{k-1}}\right) \times_{\left(\operatorname{Vec}_{X}^{r_{1}} \times \cdots \times \operatorname{Vec}_{X}^{r_{k}-r_{k-1}}\right)} \operatorname{Vec} \frac{r}{X}
$$

qui se déduit du carré commutatif

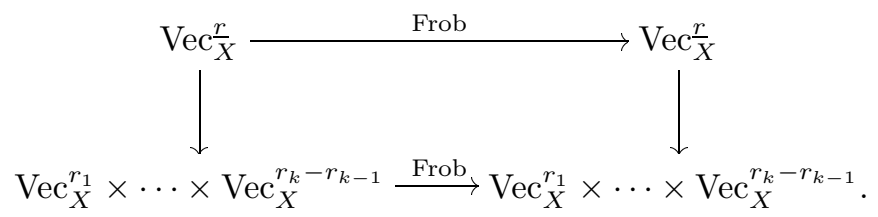

Ce problème est donc représenté par un morphisme fini, surjectif et radiciel.

Enfin, connaissant $\mathcal{E}$, on a

$$
\mathcal{E}^{\prime}=\left(\mathcal{E} \oplus \mathcal{A}_{r_{1}}^{\prime}\right) / \mathcal{A}_{r_{1}}, \quad \mathcal{E}^{\prime \prime}=\operatorname{Ker}\left[\mathcal{E}^{\prime} \rightarrow \mathcal{A}_{r} \rightarrow \mathcal{A}_{r} / \mathcal{A}_{r}^{\prime} \otimes^{\tau-1}\left(\bigotimes_{\substack{t \in \underline{r} \\ t<r}} \mathcal{L}_{t}\right)\right]
$$

et toutes les autres données qui entrent dans la composition des chtoucas itérés de type $\underline{r}$ se construisent d'une et d'une seule façon.

(iii) En effet, si $\mu^{-}\left(\mathcal{A}_{s}\right) \geq \mu+\mu^{+}\left(\mathcal{A}_{s^{+}}\right), s \in \underline{r}, s<r$, et si la constante $\mu$ est assez grande, il y a une unique possibilité pour $\mathcal{E}$ muni d'une filtration de quotients successifs les $\mathcal{A}_{s}, s \in \underline{r}$, à savoir $\mathcal{E}=\bigoplus_{s \in \underline{r}} \mathcal{A}_{s}$.

Ceci joint à la démonstration de (ii) prouve le résultat annoncé.

d) Sous-objets. Troncatures. Soient $K$ un corps contenant $\mathbb{F}_{q}, S$ le spectre de $K, r$ un entier positif, $\underline{r}=\left(r_{1}, \ldots, r_{k}\right)$ une partition de $r$, avec donc $0<r_{1}<$

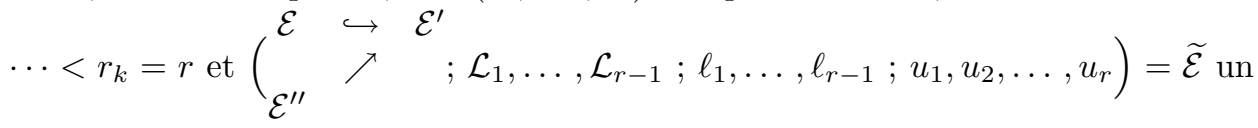
chtouca itéré de type $\underline{r}$ au-dessus de $S$.

Comme on a déjà dit, chaque $\mathcal{L}_{s}, s \notin \underline{r}$, muni de la section inversible $\ell_{s}$ peut être identifié au fibré trivial $\mathcal{O}_{S}$ muni de la section 1. D'autre part, pour tout $s \in \underline{r}$, $s<r$, la section $\ell_{s}$ est nulle et on peut toujours choisir une trivialisation $\mathcal{L}_{s} \cong \mathcal{O}_{S}$ de $\mathcal{L}_{s}$. 
Ceci étant posé, la donnée de la famille d'homomorphismes $u_{1}, u_{2}, \ldots, u_{r}$ est équivalente à celle de

- une filtration décroissante ${ }^{\tau} \mathcal{E}=\overline{\mathcal{E}}_{0} \nsupseteq \cdots \nsupseteq \overline{\mathcal{E}}_{s} \supseteq \cdots \nsupseteq \overline{\mathcal{E}}_{r}=0$ de ${ }^{\tau} \mathcal{E}$ par des sous-fibrés maximaux,

- une filtration croissante $0=\mathcal{E}_{0}^{\prime \prime} \varsubsetneqq \cdots \varsubsetneqq \mathcal{E}_{s}^{\prime \prime} \varsubsetneqq \cdots \varsubsetneqq \mathcal{E}_{r}^{\prime \prime}=\mathcal{E}^{\prime \prime}$ de $\mathcal{E}^{\prime \prime}$ par des sous-fibrés maximaux induisant des filtrations semblables $0=\mathcal{E}_{0}^{\prime} \varsubsetneqq \cdots \varsubsetneqq \mathcal{E}_{s}^{\prime} \varsubsetneqq$ $\cdots \varsubsetneqq \mathcal{E}_{r}^{\prime}=\mathcal{E}^{\prime}$ et $0=\mathcal{E}_{0} \varsubsetneqq \cdots \varsubsetneqq \mathcal{E}_{s} \varsubsetneqq \cdots \varsubsetneqq \mathcal{E}_{r}=\mathcal{E}$ de $\mathcal{E}^{\prime}$ et $\mathcal{E}$,

- une famille d'isomorphismes

$$
\overline{\mathcal{E}}_{s^{-}} / \overline{\mathcal{E}}_{s} \stackrel{\sim}{\longrightarrow} \mathcal{E}_{s}^{\prime \prime} / \mathcal{E}_{s^{-}}^{\prime \prime}, s \in \underline{r} .
$$

Définition 8. Avec les notations ci-dessus, on appellera sous-objet d'un chtouca itéré $\widetilde{\mathcal{E}}$ de type $\underline{r}$ au-dessus du spectre $S$ d'un corps $K$ contenant $\mathbb{F}_{q}$ tout couple $\left(\mathcal{A}, \mathcal{A}^{\prime}\right)$ constitué de deux sous-fibrés $\mathcal{A}$ et $\mathcal{A}^{\prime}$ de $\mathcal{E}$ et $\mathcal{E}^{\prime}$ ayant même rang et tels que le plongement $\mathcal{E} \hookrightarrow \mathcal{E}^{\prime}$ envoie $\mathcal{A}$ dans $\mathcal{A}^{\prime}$ et que chaque plongement $\overline{\mathcal{E}}_{s^{-}} / \overline{\mathcal{E}}_{s} \hookrightarrow$ $\mathcal{E}_{s}^{\prime} / \mathcal{E}_{s^{-}}^{\prime}, s \in \underline{r}$, envoie ${ }^{\tau} \mathcal{A} \cap \overline{\mathcal{E}}_{s^{-}} /{ }^{\tau} \mathcal{A} \cap \overline{\mathcal{E}}_{s}$ dans $\mathcal{A}^{\prime} \cap \mathcal{E}_{s}^{\prime} / \mathcal{A}^{\prime} \cap \mathcal{E}_{s^{-}}^{\prime}$.

Un tel sous-objet sera dit bon si $\mathcal{A}$ et $\mathcal{A}^{\prime}$ sont maximaux et s'il existe a $\in \underline{r}$ tel que

$$
\mathcal{E}_{a^{-}} \varsubsetneqq \mathcal{A} \subseteq \mathcal{E}_{a}, \quad \mathcal{E}_{a^{-}}^{\prime} \varsubsetneqq \mathcal{A}^{\prime} \subseteq \mathcal{E}_{a}^{\prime} .
$$

Un bon sous-objet sera dit de type I si $a=r_{1}=0^{+}$ou bien si $a>r_{1}=0^{+}$et $\operatorname{deg}\left({ }^{\tau} \mathcal{A} \cap \overline{\mathcal{E}}_{a^{-}}\right)<\operatorname{deg}\left(\mathcal{A} / \mathcal{E}_{a^{-}}\right)$, c'est-à-dire si ${ }^{\tau} \mathcal{A}+\overline{\mathcal{E}}_{a^{-}}={ }^{\tau} \mathcal{E}$.

Il sera dit de type II si a $>r_{1}=0^{+}$et $\operatorname{deg}\left({ }^{\tau} \mathcal{A} \cap \overline{\mathcal{E}}_{a^{-}}\right)=\operatorname{deg}\left(\mathcal{A} / \mathcal{E}_{a^{-}}\right)$, c'est-à-dire si ${ }^{\tau} \mathcal{A}+\overline{\mathcal{E}}_{a^{-}} \mp^{\tau} \mathcal{E}$.

Proposition 9. Soit $p:[0, r] \rightarrow \mathbb{R}_{+}$un polygone.

Il existe dans le champ algébrique $\overline{\mathrm{Cht}_{r}^{r}}$ des chtoucas itérés de type $\underline{r}$ un unique ouvert $\overline{\mathrm{Cht}_{\underline{r}}^{r, \bar{p} \leq p}}$ tel que pour tout point géométrique $\widetilde{\mathcal{E}}$ de $\overline{\mathrm{Cht}_{\underline{r}}^{r}}$ à valeurs dans le spectre $S$ d'un corps $K$ contenant $\mathbb{F}_{q}$, ce point est dans l'ouvert $\overline{\mathrm{Cht}_{\underline{r}}^{r, \bar{p}} \leq p}$ si et seulement si:

(i) Pour tout $s \in \underline{r}$, on $a$

$$
p(s)-1<\operatorname{deg} \mathcal{E}_{s}-\frac{s}{r} \operatorname{deg} \mathcal{E} \leq p(s) .
$$

(ii) Pour tout bon sous-objet $\left(\mathcal{A}, \mathcal{A}^{\prime}\right)$ de $\widetilde{\mathcal{E}}$, on a

$$
\operatorname{deg} \mathcal{A}-\frac{\operatorname{rg} \mathcal{A}}{r} \operatorname{deg} \mathcal{E} \leq p(\operatorname{rg} \mathcal{A})
$$

si $\left(\mathcal{A}, \mathcal{A}^{\prime}\right)$ est de type $\mathrm{I}$, et

$$
\operatorname{deg} \mathcal{A}-\frac{\operatorname{rg} \mathcal{A}}{r} \operatorname{deg} \mathcal{E} \leq p(\operatorname{rg} \mathcal{A})-1
$$

si $\left(\mathcal{A}, \mathcal{A}^{\prime}\right)$ est de type II.

De plus, si $\mathcal{L}$ est un fibré inversible sur $X$ de degré non nul, le quotient $\overline{\mathrm{Cht}_{r}^{r}, \bar{p} \leq p} / \mathcal{L}^{\mathbb{Z}}$ est de type fini au-dessus de $X \times X \times X^{k-1}$.

Démonstration. C'est une conséquence immédiate de la proposition 7 (ii) du paragraphe précédent et de ce qu'on sait à propos des champs de chtoucas.

Rappelons qu'étant donnée $\mu \geq 0$ une constante, un polygone $p:[0, r] \rightarrow \mathbb{R}$ est dit $\mu$-grand s'il vérifie les inégalités

$$
\left[p\left(r^{\prime}\right)-p\left(r^{\prime}-1\right)\right]-\left[p\left(r^{\prime}+1\right)-p\left(r^{\prime}\right)\right] \geq \mu, \quad 1 \leq r^{\prime}<r .
$$


Et dire qu'une propriété est vraie "pour tout polygone assez grand" signifie qu'il existe une constante $\mu$ telle que la propriété soit vraie pour tout polygone qui soit $\mu$-grand.

Lemme 10. Soit $p:[0, r] \rightarrow \mathbb{R}_{+}$un polygone qui soit 2 -grand. Soit $\widetilde{\mathcal{E}}$ un chtouca itéré de type $\underline{r}$ au-dessus du spectre $S$ d'un corps $K$ contenant $\mathbb{F}_{q}$.

On suppose que $\widetilde{\mathcal{E}}$ vérifie la propriété (i) de la proposition 9.

Alors la propriété (ii) de ladite proposition est équivalente à ce que tout sous$\operatorname{objet}\left(\mathcal{A}, \mathcal{A}^{\prime}\right)$ de $\widetilde{\mathcal{E}}$ satisfasse d'une part

$$
\operatorname{deg} \mathcal{A}-\frac{\operatorname{rg} \mathcal{A}}{r} \operatorname{deg} \mathcal{E} \leq p(\operatorname{rg} \mathcal{A})
$$

et d'autre part, pour tout $s \in \underline{r}$ vérifiant ${ }^{\tau} \mathcal{A} \cap \overline{\mathcal{E}}_{s} \neq 0$,

$$
\operatorname{deg}\left({ }^{\tau} \mathcal{E}_{s} \cap{ }^{\tau} \mathcal{A}\right)+\operatorname{deg}\left(\overline{\mathcal{E}}_{s} \cap{ }^{\tau} \mathcal{A}\right)-\frac{\operatorname{rg} \mathcal{A}}{r} \operatorname{deg} \mathcal{E} \leq p(\operatorname{rg} \mathcal{A})-1
$$

Démonstration. On remarque immédiatement que l'assertion (ii) de la proposition 9 appliquée à un bon sous-objet $\left(\mathcal{A}, \mathcal{A}^{\prime}\right)$ de $\widetilde{\mathcal{E}}$ est équivalente à l'assertion ci-dessus appliquée à ce même bon sous-objet.

La seule chose à vérifier est donc que dans l'hypothèse où l'assertion ci-dessus est satisfaite par tous les bons sous-objets, elle l'est par tous les sous-objets.

Ainsi, considérons un sous-objet $\left(\mathcal{A}, \mathcal{A}^{\prime}\right)$ de $\widetilde{\mathcal{E}}$. Pour tout $s \in \underline{r}$, notons $\mathcal{A}_{s}$ et $\mathcal{A}_{s}^{\prime}$ les images réciproques par les projections $\mathcal{E}_{s} \rightarrow \mathcal{E}_{s} / \mathcal{E}_{s^{-}}$et $\overline{\mathcal{E}}_{s}^{\prime} \rightarrow \mathcal{E}_{s}^{\prime} / \mathcal{E}_{s^{-}}^{\prime}$ des sous-fibrés maximaux de $\mathcal{E}_{s} / \mathcal{E}_{s^{-}}$et $\mathcal{E}_{s}^{\prime} / \mathcal{E}_{s^{-}}^{\prime}$ engendrés par $\mathcal{A} \cap \mathcal{E}_{s} / \mathcal{A} \cap \mathcal{E}_{s^{-}}$et $\mathcal{A}^{\prime} \cap \mathcal{E}_{s}^{\prime} / \mathcal{A}^{\prime} \cap \mathcal{E}_{s^{-}}^{\prime}$. Chaque paire $\left(\mathcal{A}_{s}, \mathcal{A}_{s}^{\prime}\right)$ définit un bon sous-objet de $\widetilde{\mathcal{E}}$ et on a

$$
\begin{aligned}
\operatorname{rg} \mathcal{A} & =\sum_{s \in \underline{r}}\left(\operatorname{rg} \mathcal{A}_{s}-\operatorname{rg} \mathcal{E}_{s^{-}}\right), \\
\operatorname{deg} \mathcal{A} & \leq \sum_{s \in \underline{r}}\left(\operatorname{deg} \mathcal{A}_{s}-\operatorname{deg} \mathcal{E}_{s^{-}}\right),
\end{aligned}
$$

d'où l'inégalité

$$
\operatorname{deg} \mathcal{A}-\frac{\operatorname{rg} \mathcal{A}}{r} \operatorname{deg} \mathcal{E} \leq \sum_{s \in \underline{r}}\left[\left(\operatorname{deg} \mathcal{A}_{s}-\frac{\operatorname{rg} \mathcal{A}_{s}}{r} \operatorname{deg} \mathcal{E}\right)-\left(\operatorname{deg} \mathcal{E}_{s^{-}}-\frac{\operatorname{rg} \mathcal{E}_{s^{-}}}{r} \operatorname{deg} \mathcal{E}\right)\right]
$$

Notant $u$ le plus grand élément de $\underline{r}$ tel que pour tout $s \leq u$ dans $\underline{r}$, on ait $\operatorname{rg} \mathcal{A}_{s}=$ $\operatorname{rg} \mathcal{E}_{s}$ et donc même $\mathcal{A}_{s}=\mathcal{E}_{s}$, le terme de droite s'écrit encore

$$
\begin{aligned}
& \left(\operatorname{deg} \mathcal{A}_{u}-\frac{\operatorname{rg} \mathcal{A}_{u}}{r} \operatorname{deg} \mathcal{E}\right) \\
& \quad+\sum_{\substack{s \in r, s>u \\
\operatorname{rg} \mathcal{A}_{s}>\operatorname{rg} \mathcal{E}_{s^{-}}}}\left[\left(\operatorname{deg} \mathcal{A}_{s}-\frac{\operatorname{rg} \mathcal{A}_{s}}{r} \operatorname{deg} \mathcal{E}\right)-\left(\operatorname{deg} \mathcal{E}_{s^{-}}-\frac{\operatorname{rg} \mathcal{E}_{s^{-}}}{r} \operatorname{deg} \mathcal{E}\right)\right] .
\end{aligned}
$$

D'après les hypothèses, il est majoré par

$$
p\left(\operatorname{rg} \mathcal{A}_{u}\right)+\sum_{\substack{s \in r, s>u \\ \operatorname{rg} \mathcal{A}_{s}>\operatorname{rg} \mathcal{E}_{s^{-}}}}\left(p\left(\operatorname{rg} \mathcal{A}_{s}\right)-p\left(\operatorname{rg} \mathcal{E}_{s^{-}}\right)+1\right)
$$

et à plus forte raison $\operatorname{par} p(\operatorname{rg} \mathcal{A})$ puisque le polygone $p$ est 2-grand. 
D'autre part, on a pour tout $s \in \underline{r}$

$$
\begin{aligned}
& \operatorname{deg}\left(\mathcal{E}_{s} \cap \mathcal{A}\right) \leq \sum_{\substack{t \in \underline{r} \\
t \leq s}}\left(\operatorname{deg} \mathcal{A}_{t}-\operatorname{deg} \mathcal{E}_{t^{-}}\right), \\
& \operatorname{deg}\left(\overline{\mathcal{E}}_{s} \cap \tau \mathcal{A}\right) \leq \operatorname{deg}\left(\overline{\mathcal{E}}_{s} \cap \tau{ }^{\tau} \mathcal{A}_{s^{+}}\right)+\sum_{\substack{t \in \underline{r} \\
t>s^{+}}}\left(\operatorname{deg} \mathcal{A}_{t}-\operatorname{deg} \mathcal{E}_{t^{-}}\right),
\end{aligned}
$$

d'où on déduit de la même façon

$$
\operatorname{deg}\left({ }^{\tau} \mathcal{E}_{s}+{ }^{\tau} \mathcal{A}\right)+\operatorname{deg}\left(\overline{\mathcal{E}}_{s} \cap{ }^{\tau} \mathcal{A}\right)-\frac{\operatorname{rg} \mathcal{A}}{r} \operatorname{deg} \mathcal{E} \leq p(\operatorname{rg} \mathcal{A})-1
$$

Corollaire 11. Soit $p:[0, r] \rightarrow \mathbb{R}_{+}$un polygone qui soit 2 -grand. Alors il existe dans le champ algébrique $\overline{\mathrm{Cht}^{r}}$ des chtoucas itérés de rang $r$ un unique ouvert $\overline{\mathrm{Cht}^{r}, \bar{p} \leq p}$ dont la trace dans chaque strate $\overline{\mathrm{Cht}_{r}^{r}}$ soit égale à $\overline{\mathrm{Cht}_{r}^{r, \bar{p}} \leq p}$.

De plus, si $\mathcal{L}$ est un fibré inversible sur $X$ de degré non nul, le quotient $\overline{\mathrm{Cht}^{r, \bar{p}} \leq p} / \mathcal{L}^{\mathbb{Z}}$ est de type fini sur $X \times X$. Il est lisse de dimension relative $2 r-2$ si $p$ est assez grand.

Démonstration. Compte tenu de la proposition 9 , on a seulement à prouver que si $\widetilde{\mathcal{E}}$ est un chtouca itéré de type $\underline{r}$ au-dessus d'un corps qui se spécialise en un chtouca itéré $\widetilde{\mathcal{F}}$ de type plus fin au-dessus d'un corps résiduel et si $\widetilde{\mathcal{F}}$ vérifie les propriétés de la proposition 9 , alors $\widetilde{\mathcal{E}}$ les vérifie également.

Or les $\overline{\mathcal{E}}_{s}, \mathcal{E}_{s}, \mathcal{E}_{s}^{\prime}, \mathcal{E}_{s}^{\prime \prime}, s \in \underline{r}$, se spécialisent en les $\overline{\mathcal{F}}_{s}, \mathcal{F}_{s}, \mathcal{F}_{s}^{\prime}, \mathcal{F}_{s}^{\prime \prime}$ et d'autre part tout bon sous-objet de $\widetilde{\mathcal{E}}$ se spécialise en un sous-objet de $\widetilde{\mathcal{F}}$.

On conclut d'après le lemme 10.

Quant à la lissité dès lors que $p$ est assez grand, elle résulte de la proposition 7 (iii) du paragraphe précédent.

Enonçons:

Théorème 12. Soient $p:[0, r] \rightarrow \mathbb{R}_{+}$un polygone qui soit 2 -grand et $\mathcal{L}$ un fibré inversible sur $X$ de degré non nul.

Alors le champ $\overline{\mathrm{Cht}^{r, \bar{p}} \leq p} / \mathcal{L}^{\mathbb{Z}}$ est propre (et en particulier séparé) au-dessus de $X \times X$.

Démonstration. On sait déjà que ce champ est de type fini. Il suffit donc de vérifier le critère valuatif de propreté, ce qui fait l'objet des paragraphes suivants.

\section{VÉRIFICATION DU CRITÈRE VALUATIF DE PROPRETÉ}

Pour la démonstration, nous allons nous inspirer de celle de Drinfeld en rang 2 (voir le paragraphe 3 de [3]).

a) $\varphi$-réseaux itérés dans les $\varphi$-espaces. Etant donné $A$ un anneau de valuation discrète contenant $\mathbb{F}_{q}$, on notera $K_{A}$ le corps des fractions de $A, \kappa_{A}$ son corps résiduel, $\pi_{A}$ un élément uniformisant et $\operatorname{deg}_{A}$ sa valuation.

On notera aussi $A_{X}$ l'anneau local du schéma $X \otimes A$ en le point générique de la fibre spéciale $X \otimes \kappa_{A}$. Ainsi $A_{X}$ est un anneau de valuation discrète dont $\pi_{A}$ est également élément uniformisant. Si $F$ désigne le corps des fonctions de la courbe 
$X$, on voit que le corps des fractions de $A_{X}$ s'identifie au corps des fractions de $F \otimes K_{A}$ et le corps résiduel de $A_{X}$ au corps des fractions de $F \otimes \kappa_{A}$.

On notera encore $\tau$ les endomorphismes de $A_{X}, K_{A_{X}}$ ou de leurs complétés $\widehat{A_{X}}, K_{\widehat{A_{X}}}$ induits par le produit tensoriel $\operatorname{Id}_{F} \otimes \operatorname{Frob}_{A}$. On remarque que pour tout scalaire $a \in K_{A_{X}}\left[\operatorname{resp} . K_{\widehat{A_{X}}}\right]$, on a $\operatorname{deg}_{A_{X}}(\tau(a))=q \operatorname{deg}_{A_{X}}(a)\left[\right.$ resp. $\operatorname{deg}_{\widehat{A_{X}}}(\tau(a))=$ $\left.q \operatorname{deg}_{\widehat{A_{X}}}(a)\right]$.

Considérons $(V, \varphi)$ un $\varphi$-espace de dimension $r$ sur $K_{A_{X}}$ c'est-à-dire la donnée d'un espace vectoriel $V$ de dimension $r$ sur $K_{A_{X}}$ et d'un isomorphisme $\tau^{*} V=$ ${ }^{\tau} V \stackrel{\sim}{\longrightarrow} V$ ou, ce qui revient au même, d'une application $\tau$-linéaire $\varphi: V \rightarrow V$ telle que l'image de $\varphi$ engendre $V$ (voir [2], paragraphe 2).

Rappelons qu'un réseau de $V$ [resp. de $\widehat{V}=V \otimes_{K_{A_{X}}} K_{\widehat{A_{X}}}$ ] est un sous-module de type fini sur $A_{X}$ [resp. $\left.\widehat{A_{X}}\right]$ qui engendre $V$ [resp. $\left.\widehat{V}\right]$ comme espace vectoriel; un tel réseau est nécessairement libre de rang $r$ comme module. On remarque que l'application $M \longmapsto M \otimes_{A_{X}} \widehat{A_{X}}$ définit une bijection de l'ensemble des réseaux de $V$ sur l'ensemble des réseaux de $\widehat{V}$.

Nous allons introduire une notion de " $\varphi$-réseau itéré" qui généralise celle de $\varphi$ réseau définie par Drinfeld dans le cas du rang $r=2$ (voir le paragraphe 3 de $[3])$ :

Définition 1. Soient $A$ un anneau de valuation discrète contenant $\mathbb{F}_{q},(V, \varphi)$ un $\varphi$-espace de dimension $r$ sur $K_{A_{X}}$ et $u:{ }^{\tau} V \rightarrow V$ l'isomorphisme associé.

On appellera $\varphi$-réseau itéré dans $V$ (relativement à une famille d'entiers $d_{1}$, $\left.d_{2}, \ldots, d_{r-1} \geq 0\right)$ tout réseau $M$ de $V$ tel que, si on note

$$
u_{s}=\left(\prod_{1 \leq t<s} \pi_{A}^{d_{t}(s-t)}\right)^{-(q-1)} \Lambda^{s} u, \quad 1 \leq s \leq r,
$$

les deux propriétés suivantes sont vérifiées:

(i) Pour n'importe quel choix de base de $M$ sur $A_{X}$, le point $\left(u_{1}, u_{2}, \ldots, u_{r}\right.$; $\left.\pi_{A}^{d_{1}(q-1)}, \ldots, \pi_{A}^{d_{r-1}(q-1)}\right)$ de $\Omega_{(r)}^{r}\left(K_{A_{X}}\right)$ se prolonge en un point de $\Omega^{r}\left(A_{X}\right)$.

(ii) Pour tout $s, 1 \leq s \leq r$, la réduction modulo $\pi_{A}$ de l'homomorphisme

$$
u_{s}: \tau\left(\prod_{1 \leq t<s} \pi_{A}^{-d_{t}(s-t)} \Lambda^{s} M\right) \longrightarrow \prod_{1 \leq t<s} \pi_{A}^{-d_{t}(s-t)} \Lambda^{s} M
$$

est telle que son noyau et le transformé par $\tau$ de son image soient en somme directe (autrement dit, que toutes ses puissances aient le même rang non nul).

Bien sûr, on appellera type d'un tel $\varphi$-réseau itéré $M$ relativement à une famille d'entiers $d_{1}, d_{2}, \ldots, d_{r-1} \geq 0$ la partition $\underline{r}=\left(r_{1}, \ldots, r_{k}\right)$ telle que $r_{k}=r$ et, pour $1 \leq s<r$,

$$
s \in \underline{r} \Longleftrightarrow d_{s} \geq 1 .
$$

Un $\varphi$-réseau itéré de type $(r)$ c'est-à-dire tel que la famille associée $d_{1}, \ldots, d_{r-1}$ soit nulle sera aussi appelé simplement un $\varphi$-réseau.

D'autre part, on dispose en un sens évident de la notion de $\varphi$-espace (de dimension finie) sur $K_{\widehat{A_{X}}}$ et, dans un tel $\varphi$-espace, de celles de $\varphi$-réseau itéré et de $\varphi$-réseau. On note que si $(V, \varphi)$ est un $\varphi$-espace sur $K_{A_{X}}, \widehat{V}=V \otimes_{K_{A_{X}}} K_{\widehat{A_{X}}}$ est un $\varphi$-espace et l'application $M \longmapsto M \otimes_{A_{X}} \widehat{A_{X}}$ est une bijection de l'ensemble des $\varphi$-réseaux itérés [resp. des $\varphi$-réseaux] de $V$ sur l'ensemble de ceux de $\widehat{V}$. 
Considérons $(\widehat{V}, \varphi)$ un $\varphi$-espace sur $K_{\widehat{A_{X}}}$ et $\widehat{M}$ un $\varphi$-réseau itéré de type $\underline{r}=$ $\left(r_{1}, \ldots, r_{k}\right)$ dans $\widehat{V}$ relativement à une famille $d_{1}, d_{2}, \ldots, d_{r-1} \geq 0$. Posons

$$
\begin{aligned}
& \widehat{M}_{r_{1}}=\bigcap_{n \geq 1} \widehat{A_{X}} \varphi^{n}(\widehat{M}), \quad \widehat{V}_{r_{1}}=\widehat{M}_{r_{1}} \otimes_{\widehat{A_{X}}} K_{\widehat{A_{X}}}, \\
& \widehat{M}_{r_{2}}=\bigcap_{n \geq 1} \widehat{A_{X}} \varphi^{n}\left(\pi_{A}^{-d r_{1}} \widehat{M} / \widehat{M}_{r_{1}}\right), \widehat{V}_{r_{2}} / \widehat{V}_{r_{1}}=\widehat{M}_{r_{2}} \otimes_{\widehat{A_{X}}} K_{\widehat{A_{X}}}, \\
& \text { etc. }
\end{aligned}
$$

Ainsi on associe à $\widehat{M}$ une filtration croissante $O=\widehat{V}_{0} \varsubsetneqq \widehat{V}_{r_{1}} \varsubsetneqq \widehat{V}_{r_{2}} \varsubsetneqq \cdots \varsubsetneqq \widehat{V}_{r_{k}}=$ $\widehat{V}$ de $\widehat{V}$ par des $\varphi$-espaces, telle que chaque quotient $\widehat{V}_{s} / \widehat{V}_{s^{-}}, s \in \underline{r}$, soit muni d'un $\varphi$-réseau $\widehat{M}_{s}$.

Proposition 2. Soient $A$ un anneau de valuation discrète contenant $\mathbb{F}_{q}$ et $(\widehat{V}, \varphi)$ un $\varphi$-espace sur $K_{\widehat{A_{X}}}$.

Alors:

(i) Il existe dans $\widehat{V}$ au plus un $\varphi$-réseau.

(ii) Plus généralement, si $0=\widehat{V}_{0} \varsubsetneqq \widehat{V}_{r_{1}} \varsubsetneqq \cdots \varsubsetneqq \widehat{V}_{r_{k}}=\widehat{V}$ est une filtration de $\widehat{V}$ par des $\varphi$-espaces et si $d_{1}, d_{2}, \ldots, d_{r-1}$ est une famille d'entiers telle que $s \in \underline{r}=\left(r_{1}, \ldots, r_{k}\right) \Rightarrow d_{s} \geq 1$ et $s \notin \underline{r} \Rightarrow d_{s}=0$, il existe dans $\widehat{V}$ au plus un $\varphi-$ réseau itéré relativement à $d_{1}, \ldots, d_{r-1}$ dont la filtration de $\widehat{V}$ associée soit égale $\grave{a}\left(\widehat{V}_{s}\right)_{s \in \underline{r}}$.

(iii) Dans la situation de (ii), supposons que chaque quotient $\widehat{V}_{s} / \widehat{V}_{s^{-}}, s \in \underline{r}$, admette un $\varphi$-réseau. Alors, si les $d_{s}, s \in \underline{r}$, sont assez grands, le problème posé en (ii) admet une solution.

Démonstration. (i) En effet, un tel $\varphi$-réseau est nécessairement l'ensemble des $v \in$ $\widehat{V}$ tels que le sous-module engendré sur $\widehat{A_{X}}$ par les $\varphi^{n}(v), n \geq 1$, soit de type fini.

(ii) Soient $\widehat{M}$ et $\widehat{N}$ deux tels $\varphi$-réseaux itérés. On sait déjà d'après (i) que pour tout $s \in \underline{r}$, on a $\widehat{M} \cap \widehat{V}_{s} / \widehat{M} \cap \widehat{V}_{s^{-}}=\widehat{N} \cap \widehat{V}_{s} / \widehat{N} \cap \widehat{V}_{s^{-}}$.

Montrons par récurrence descendante sur les $s \in \underline{r}$ que

$$
\widehat{M} / \widehat{M} \cap \widehat{V}_{s^{-}}=\widehat{N} / \widehat{N} \cap \widehat{V}_{s^{-}} .
$$

Supposant que l'on sait déjà $\widehat{M} / \widehat{M} \cap \widehat{V}_{s}=\widehat{N} / \widehat{N} \cap \widehat{V}_{s}$, considérons $m \in \widehat{M} / \widehat{M} \cap \widehat{V}_{s^{-}}$et $n \in \widehat{N} / \widehat{N} \cap \widehat{V}_{s^{-}}$deux éléments de $\widehat{V} / \widehat{V}_{s^{-}}$qui ont même réduction dans $\widehat{M} / \widehat{M} \cap \widehat{V}_{s}=$ $\widehat{N} / \widehat{N} \cap \widehat{V}_{s} \subseteq \widehat{V} / \widehat{V}_{s}$. Alors l'élément $m-n \in \widehat{V}_{s} / \widehat{V}_{s^{-}}$est tel que le sous-module engendré sur $\widehat{A_{X}}$ par ses transformés par les puissances de $\left(\prod_{t \in \underline{s}} \pi_{A}^{-d_{t}}\right) \varphi$ soit de type fini. Par conséquent, $m-n$ est comme voulu dans $\widehat{M} \cap \widehat{V}_{s} / \widehat{M} \cap \widehat{V}_{s^{-}}=\widehat{N} \cap \widehat{V}_{s} / \widehat{N} \cap \widehat{V}_{s^{-}}$.

(iii) Pour tout $s \in \underline{r}$, soit $\widehat{M}_{s}$ le $\varphi-$ réseau de $\widehat{V}_{s} / \widehat{V}_{s^{-}}$. On peut toujours le relever en un module $\widehat{M}_{s}^{\prime} \subseteq \widehat{V}_{s}$ libre de même rang sur $\widehat{A}_{X}$.

Il est clair que si les $d_{s}, s \in \underline{r}$, sont assez grands, le réseau $\widehat{M}=\sum_{s \in \underline{r}}\left(\prod_{\substack{t \in \underline{r} \\ t<s}} \pi_{A}^{d_{t}}\right) \widehat{M}_{s}^{\prime}$ répond à la question posée.

Nous allons maintenant montrer que, quitte à étendre la base, il existe des $\varphi^{-}$ réseaux itérés dans n'importe quel $\varphi$-espace. 
Lemme 3 (Drinfeld). Soient $A$ un anneau de valuation discrète contenant $\mathbb{F}_{q}$ et $(\widehat{V}, \varphi)$ un $\varphi$-espace sur $K_{\widehat{A_{X}}}$.

Alors, quitte à remplacer A par une extension finie totalement ramifiée, il existe dans $\widehat{V}$ un réseau $\widehat{M}$ stabilisé par $\varphi$ et tel que l'endomorphisme induit $\bar{\varphi}$ : $\widehat{M} / \pi_{A} \widehat{M} \rightarrow \widehat{M} / \pi_{A} \widehat{M}$ ne soit pas nilpotent.

Démonstration. On prétend qu'il existe sur $\widehat{V}$ une valuation $\operatorname{deg}_{\varphi}$ telle que $\operatorname{deg}_{\varphi}(\varphi(v))=q \operatorname{deg}_{\varphi}(v), \forall v \in \widehat{V}$.

En effet, il suffit pour s'en convaincre de partir d'une valuation quelconque deg sur $V$ et de remarquer qu'il existe une constante $C>0$ telle que pour tout $v \in \widehat{V}$ on ait

$$
|\operatorname{deg}(\varphi(v))-q \operatorname{deg}(v)| \leq C
$$

si bien qu'on peut définir

$$
\operatorname{deg}_{\varphi}(v)=\lim _{n \mapsto+\infty} q^{-n} \operatorname{deg}\left(\varphi^{n}(v)\right) .
$$

Comme le corps $K_{\widehat{A_{X}}}$ est complet, il existe des réels $c_{1}, c_{2}, \ldots, c_{r}$ et une base $v_{1}, \ldots, v_{r}$ de $\widehat{V}$ tels que pour tout $v \in \widehat{V}$ de coordonnées $a_{1}, a_{2}, \ldots, a_{r}$, on ait

$$
\operatorname{deg}_{\varphi}(v)=\min _{1 \leq i \leq r}\left\{c_{i}+\operatorname{deg}_{\widehat{A_{X}}}\left(a_{i}\right)\right\} .
$$

Comme d'autre part $\operatorname{deg}_{\varphi}\left(\varphi\left(v_{j}\right)\right)=q \operatorname{deg}_{\varphi}\left(v_{j}\right), 1 \leq j \leq r$, on voit que si $\left(a_{i j}\right)$ désigne la matrice de $\varphi$ dans la base considérée, on a

$$
q c_{j}=\min _{1 \leq i \leq r}\left\{c_{i}+\operatorname{deg}_{\widehat{A_{X}}}\left(a_{i j}\right)\right\}, \quad 1 \leq j \leq r .
$$

Les $\operatorname{deg}_{\widehat{A_{X}}}\left(a_{i j}\right)$ étant des entiers, il résulte de ces relations que $c_{1}, c_{2}, \ldots, c_{r}$ sont des rationnels. Et si $e \geq 1$ désigne un de leurs dénominateurs communs, on obtient que $\operatorname{deg}_{\varphi}$ prend ses valeurs dans $\frac{1}{e} \mathbb{Z}$.

Puis, quitte à remplacer $A$ par $A\left[\pi_{A}^{1 / e}\right]$, on peut supposer que la valuation $\operatorname{deg}_{\varphi}$ atteint la valeur 0 .

Alors le réseau $\widehat{M}=\left\{v \in \widehat{V}, \operatorname{deg}_{\varphi}(v) \geq 0\right\}$ répond à la question posée.

Proposition 4. Soient $A$ un anneau de valuation discrète contenant $\mathbb{F}_{q}$ et $(V, \varphi)$ $[$ resp. $(\widehat{V}, \varphi)]$ un $\varphi$-espace sur $K_{A_{X}}\left[\right.$ resp. $\left.K_{\widehat{A_{X}}}\right]$.

Alors, quitte à remplacer A par une extension finie totalement ramifiée, il existe dans $V[$ resp. $\widehat{V}]$ un $\varphi$-réseau itéré.

Démonstration. Il suffit de considérer le cas d'un $\varphi$-espace $(\widehat{V}, \varphi)$ sur $K_{\widehat{A_{X}}}$.

D'après le lemme 3 et quitte à étendre la base $A$, il existe dans $\widehat{V}$ un réseau $\widehat{N}_{1}$ stable par $\varphi$ et tel que $\widehat{M}_{r_{1}}=\bigcap_{n \geq 1} \widehat{A_{X}} \varphi^{n}\left(\widehat{N}_{1}\right)$ soit un module libre de rang $r_{1} \geq 1$ sur $\widehat{A_{X}}$. Alors $\widehat{M}_{r_{1}}$ est un $\varphi$-réseau de $\widehat{V}_{r_{1}}=\widehat{M}_{r_{1}} \otimes_{\widehat{A_{X}}} K_{\widehat{A_{X}}}$.

Puis en recommençant ce processus autant de fois que nécessaire, on construit dans $\widehat{V}$ une filtration croissante par des $\varphi$-espaces $\widehat{V}_{s}, s \in \underline{r}$, telle que chaque quotient $\widehat{V}_{s} / \widehat{V}_{s^{-}}$admette un $\varphi$-réseau. On conclut d'après la proposition 2 (iii).

Terminons ce paragraphe en introduisant quelques notations et notions nouvelles dont nous aurons besoin. 
Considérons $A$ un anneau de valuation discrète contenant $\mathbb{F}_{q},(V, \varphi)$ un $\varphi$-espace de dimension $r$ sur $K_{A_{X}}, M$ un $\varphi$-réseau itéré de type $\underline{r}=\left(r_{1}, \ldots, r_{k}\right)$ dans $V$ et $\widehat{M}=M \otimes_{A_{X}} \widehat{A_{X}}$ le $\varphi$-réseau itéré correspondant dans $\bar{V}=V \otimes_{K_{A_{X}}} K_{\widehat{A_{X}}}$.

On notera $V^{M}=M / \pi_{A} M=\widehat{M} / \pi_{A} \widehat{M}=\widehat{V}^{\widehat{M}}$. C'est un espace vectoriel de dimension $r$ sur le corps résiduel $\kappa_{A_{X}}$. D'après la définition 1 du présent paragraphe combinée à la proposition $1 \mathrm{du}$ paragraphe $1 \mathrm{a}$, on dispose

- dans $V^{M}$ d'une filtration croissante $0=V_{0}^{M} \varsubsetneqq V_{r_{1}}^{M} \varsubsetneqq \cdots \varsubsetneqq V_{s}^{M} \varsubsetneqq \cdots \varsubsetneqq$ $V_{r}^{M}=V^{M}$ par des sous-espaces vectoriels $V_{s}^{M}, s \in \underline{r}$, de dimension $s$,

- dans ${ }^{\tau} V^{M}$ d'une filtration décroissante ${ }^{\tau} V^{M}=\bar{V}_{0}^{M} \supseteq \cdots \supseteq \bar{V}_{s}^{M} \supseteq \cdots \supseteq$ $\bar{V}_{r}^{M}=0$ par des sous-espaces vectoriels $\bar{V}_{s}^{M}, s \in \underline{r}$, de codimension $s$,

- pour tout $s, s \in \underline{r}$, d'un isomorphisme

$$
\bar{V}_{s^{-}}^{M} / \bar{V}_{s}^{M} \stackrel{\sim}{\longrightarrow} V_{s}^{M} / V_{s^{-}}^{M} .
$$

De plus, la condition (ii) de la définition 1 est équivalente à ce que, pour tout $s \in \underline{r}$, $\bar{V}_{s}^{M}$ et ${ }^{\tau} V_{s}^{M}$ soient en somme directe dans ${ }^{\tau} V^{M}$.

On remarque que si $0=\widehat{V}_{0}(M) \varsubsetneqq \cdots \varsubsetneqq \widehat{V}_{s}(M) \varsubsetneqq \cdots \varsubsetneqq \widehat{V}_{r}(M)=\widehat{V}$ est la filtration de $\widehat{V}$ par des $\varphi$-espaces $\widehat{V}_{s}(M), s \in \underline{r}$, de dimension $s$, canoniquement associée au $\varphi$-réseau itéré $M$, alors pour tout $s \in \underline{r}$, on a

$$
V_{s}^{M}=M \cap \widehat{V}_{s}(M) / \pi_{A}\left(M \cap \widehat{V}_{s}(M)\right) .
$$

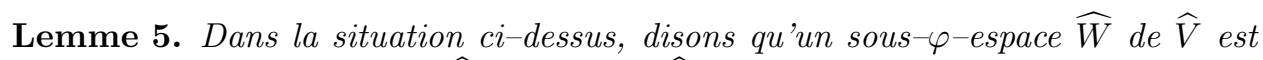
compatible avec la filtration $\left(\widehat{V}_{s}(M)\right)_{s \in \underline{r}}$ de $\widehat{V}$ canoniquement associée au $\varphi-r e ́ s e a u$ itéré $M$ s'il existe un $s \in \underline{r}$ tel que $\widehat{V}_{s^{-}}(M) \varsubsetneqq \widehat{W} \subseteq \widehat{V}_{s}(M)$.

D'autre part, un sous-espace $W$ de $V^{M}=\widehat{M} / \pi_{A} \widehat{M}$ sera dit bon s'il existe un $s \in \underline{r}$ tel que $V_{s^{-}}^{M} \varsubsetneqq W \subseteq V_{s}^{M}$ et que l'isomorphisme $\bar{V}_{s^{-}}^{M} / \bar{V}_{s}^{M} \stackrel{\sim}{\longrightarrow} V_{s}^{M} / V_{s^{-}}^{M}$ induise un isomorphisme $\bar{V}_{s^{-}}^{M} \cap^{\tau} W \stackrel{\sim}{\longrightarrow} W / V_{s^{-}}^{M}$.

Alors l'application

$$
\widehat{W} \longmapsto \widehat{W} \widehat{M}=\widehat{M} \cap \widehat{W} / \pi_{A}(\widehat{M} \cap \widehat{W})
$$

induit une bijection de l'ensemble des sous- $\varphi$-espaces compatibles de $\widehat{V}$ sur l'ensemble des bons sous-espaces de $V^{M}$.

Démonstration. Soit $\left(d_{1}, \ldots, d_{r-1}\right)$ la suite d'entiers relativement à laquelle $M$ est un $\varphi$-réseau itéré.

Considérons $W$ un bon sous-espace de $V^{M}=\widehat{M} / \pi_{A} \widehat{M}$ avec donc $V_{s^{-}}^{M} \varsubsetneqq W \subseteq$ $V_{s}^{M}$ pour un certain $s \in \underline{r}$. Choisissons un $\widehat{A_{X}}-$ module de type fini $\widehat{N}$ tel que $\widehat{N} \subseteq \widehat{M}, \widehat{N} \supseteq \widehat{M} \cap \widehat{V}_{s^{-}}(M)$ et $\widehat{N} / \pi_{A} \widehat{N}=W$

Soit $\widehat{W}$ l'unique sous-espace de $\widehat{V}$ qui contienne $\widehat{V}_{s^{-}}(M)$ et tel que $\widehat{W} / \widehat{V}_{s^{-}}(M)$ soit engendré sur $K_{\widehat{A_{X}}}$ par l'intersection

$$
\bigcap_{n \geq 1} \widehat{A_{X}}\left(\left(\prod_{\substack{t \in r \\ t<s}} \pi_{A}^{-d_{t}}\right) \varphi\right)^{n}\left(\widehat{N} / \widehat{M} \cap \widehat{V}_{s^{-}}(M)\right) .
$$

Alors $\widehat{W}$ est l'unique antécédent de $W$ par l'application considérée.

Proposition 6. Soient $A$ un anneau de valuation discrète contenant $\mathbb{F}_{q},(V, \varphi)$ un $\varphi$-espace de dimension $r$ sur $K_{A_{X}}, M$ un $\varphi$-réseau itéré dans $V$ relativement à 
une famille d'entiers $d_{1}, d_{2}, \ldots, d_{r-1} \geq 0$ et $\widehat{W}$ un sous- $\varphi$-espace de $\widehat{V}$ compatible avec la filtration $\left(\widehat{V}_{s}(M)\right)_{s \in \underline{r}}$ canoniquement associée à $M$.

Alors, $\widehat{W^{M}}$ désignant le bon sous-espace de $V^{M}=M / \pi_{A} M$ correspondant $\grave{a} \widehat{W}$, $M^{\prime}=\operatorname{Ker}\left[M \rightarrow V^{M} / \widehat{W}^{\widehat{M}}\right]$ est un $\varphi$-réseau itéré dans $V$.

Il l'est relativement à la famille $\left(d_{1}, \ldots, d_{r^{\prime}-1}, d_{r^{\prime}}+1, d_{r^{\prime}+1}, \ldots, d_{r-1}\right)$ où $r^{\prime}$ désigne la dimension de $\widehat{W}$ sur $K_{\widehat{A_{X}}}$.

Enfin, la filtration de $\widehat{V}$ associée à $M^{\prime}$ est égale à la réunion de celle $\left(\widehat{V}_{s}(M)\right)_{s \in \underline{r}}$ associée à $M$ et de l'élément $\widehat{W}$.

Dans la situation de la proposition 6, on dira que $M^{\prime}$ est le transformé de $M$ par $\widehat{W}$ ou $\widehat{W}^{\widehat{M}}$. On pourra dire aussi que $M$ est le transformé réciproque de $M^{\prime}$ par $\widehat{W}$.

Notant $\underline{r}^{\prime}$ la réunion de $\underline{r}$ et de l'élément $r^{\prime}=\operatorname{dim} \widehat{W}=\operatorname{dim} \widehat{W^{\widehat{M}}}$, la filtration croissante canonique $\left(V_{s}^{M^{\prime}}\right)_{s \in r^{\prime}}$ de $V^{M^{\prime}}=M^{\prime} / \pi_{A} M^{\prime}$ est telle que $V_{r^{\prime}}^{M^{\prime}} \cong \widehat{W^{M}}$, $V_{s}^{M^{\prime}}=V_{s}^{M}$ pour $s<r^{\prime}$ et $V_{s}^{M^{\prime}} / V_{r^{\prime}}^{M^{\prime}}=V_{s}^{M} / \widehat{W}^{\widehat{M}}$ pour $s>r^{\prime}$, et la filtration décroissante canonique $\left(\bar{V}_{s}^{M^{\prime}}\right)_{s \in \underline{r}^{\prime}}$ de ${ }^{\tau} V^{M^{\prime}}$ est telle que $\bar{V}_{r^{\prime}}^{M^{\prime}}={ }^{\tau}\left(V^{M} / \widehat{W^{M}}\right)$, $\bar{V}_{s}^{M^{\prime}}=\bar{V}_{s}^{M}$ pour $s>r^{\prime}$ et $\bar{V}_{s}^{M^{\prime}} / \bar{V}_{r^{\prime}}^{M^{\prime}}={ }^{\tau} \widehat{W}^{\widehat{M}} \cap \bar{V}_{s}^{M}$ pour $s<r^{\prime}$.

b) Chtoucas dégénérés associés aux $\varphi$-réseaux itérés. Nous allons nous servir du lemme suivant:

Lemme 7. Soit $A$ un anneau de valuation discrète contenant $\mathbb{F}_{q}$. Alors le foncteur qui à tout $\mathcal{O}_{X \otimes A}$-Module localement libre sur $X \otimes A$ associe d'une part sa restriction à la fibre générique $X \otimes K_{A}$ et d'autre part sa fibre au-dessus de Spec $A_{X}$ est une équivalence de catégories, c'est-à-dire admet un foncteur quasi-inverse qu'on notera $(\mathcal{E}, M) \longmapsto \mathcal{E}(M)$.

Dans tout ce qui suit, on fixera un chtouca $\left(\begin{array}{cc}\mathcal{E} & \hookrightarrow \mathcal{E}^{\prime} \\ \tau \mathcal{E}=\mathcal{E}^{\prime \prime} & \nearrow\end{array}\right)$ de $\operatorname{rang} r \geq 1 \mathrm{au}-$ dessus du point générique Spec $K_{A}$ d'un anneau de valuation discrète $A$ contenant $\mathbb{F}_{q}$. Sa fibre générique est un $\varphi$-espace $V$ de dimension $r$ sur $K_{A_{X}}$.

D'après le lemme 7 ci-dessus et la définition 1 du paragraphe $2 \mathrm{a}$, on peut associer à tout $\varphi$-réseau itéré $M$ de $V$

- un $\mathcal{O}_{X \otimes A^{-}}$Module $\mathcal{E}(M)$ localement libre de rang $r$,

- une modification $\left(\begin{array}{ccc}\mathcal{E}(M) & \hookrightarrow & \mathcal{E}^{\prime}(M) \\ \mathcal{E}^{\prime \prime}(M) & \nearrow\end{array}\right)$ de celui-ci,

- une famille d'homomorphismes

$$
{ }^{\tau} \Lambda^{s} \mathcal{E}(M) \longrightarrow \Lambda^{s} \mathcal{E}^{\prime \prime}(M), \quad 1 \leq s \leq r .
$$

L'ensemble de ces données pourra être appelé le chtouca dégénéré induit par $M$. Les restrictions $\mathcal{E}^{M}=\mathcal{E}(M) / \pi_{A} \mathcal{E}(M), \mathcal{E}^{\prime M}=\mathcal{E}^{\prime}(M) / \pi_{A} \mathcal{E}^{\prime}(M)$ et $\mathcal{E}^{\prime \prime M}=$ $\mathcal{E}^{\prime \prime}(M) / \pi_{A} \mathcal{E}^{\prime \prime}(M)$ à la fibre spéciale $X \otimes \kappa_{A}$ sont des fibrés (localement libres) de rang $r$ dont les fibres génériques s'identifient à $V^{M}=M / \pi_{A} M$.

Si $\underline{r}=\left(r_{1}, \ldots, r_{k}\right)$ désigne le type du $\varphi$-réseau itéré $M$, les filtrations $0=V_{0}^{M} \varsubsetneqq$ $V_{r_{1}}^{M} \varsubsetneqq \cdots \varsubsetneqq V_{s}^{M} \varsubsetneqq \cdots \varsubsetneqq V_{r}^{M}=V^{M}$ et ${ }^{\tau} V^{M}=\bar{V}_{0}^{M} \supseteq \cdots \nsupseteq \bar{V}_{s}^{M} \supseteq \cdots \nsupseteq \bar{V}_{r}^{M}=0$ 
induisent des filtrations

$$
\begin{aligned}
& 0=\mathcal{E}_{0}^{M} \varsubsetneqq \mathcal{E}_{r_{1}}^{M} \varsubsetneqq \cdots \varsubsetneqq \mathcal{E}_{s}^{M} \varsubsetneqq \cdots \varsubsetneqq \mathcal{E}_{r}^{M}=\mathcal{E}^{M}, \\
& 0=\mathcal{E}_{0}^{\prime M} \varsubsetneqq \mathcal{E}_{r_{1}}^{\prime M} \varsubsetneqq \cdots \varsubsetneqq \mathcal{E}_{s}^{\prime M} \varsubsetneqq \cdots \varsubsetneqq \mathcal{E}_{r}^{\prime M}=\mathcal{E}^{\prime M} \text {, } \\
& 0=\mathcal{E}_{0}^{\prime \prime M} \varsubsetneqq \mathcal{E}_{r_{1}}^{\prime \prime M} \varsubsetneqq \cdots \varsubsetneqq \mathcal{E}_{s}^{\prime \prime M} \varsubsetneqq \cdots \varsubsetneqq \mathcal{E}_{r}^{\prime M}=\mathcal{E}^{\prime \prime M} \\
& \text { et }{ }^{\tau} \mathcal{E}^{M}=\overline{\mathcal{E}}_{0}^{M} \supseteq \overline{\mathcal{E}}_{r_{1}}^{M} \supseteq \cdots \supseteq \overline{\mathcal{E}}_{s}^{M} \supseteq \cdots \supseteq \overline{\mathcal{E}}_{r}^{M}=0
\end{aligned}
$$

de $\mathcal{E}^{M}, \mathcal{E}^{M}, \mathcal{E}^{\prime \prime M}$ et ${ }^{\tau} \mathcal{E}^{M}$ par des sous-fibrés maximaux.

De plus on dispose, pour tout $s \in \underline{r}$, d'un isomorphisme au-dessus d'un ouvert non vide de $X \otimes \kappa_{A}$

$$
\overline{\mathcal{E}}_{s^{-}}^{M} / \overline{\mathcal{E}}_{s}^{M} \stackrel{\sim}{\longrightarrow} \mathcal{E}_{s}^{\prime \prime M} / \mathcal{E}_{s^{-}}^{\prime \prime M} .
$$

On sait enfin que pour tout $s \in \underline{r}, \overline{\mathcal{E}}_{s}^{M}$ et ${ }^{\tau} \mathcal{E}_{s}^{M}$ sont d'intersection nulle dans ${ }^{\tau} \mathcal{E}^{M}$ et que le quotient ${ }^{\tau} \mathcal{E}^{M} /\left(\overline{\mathcal{E}}_{s}^{M} \oplus{ }^{\tau} \mathcal{E}_{s}^{M}\right)$ est de torsion.

Proposition 8. Soit $M$ un $\varphi$-réseau itéré de type $\underline{r}=\left(r_{1}, \ldots, r_{k}\right)$ dans $V$.

(i) Pour tout $s \in \underline{r}$, le quotient ${ }^{\tau} \mathcal{E}^{M} /\left(\overline{\mathcal{E}}_{s}^{M} \oplus^{\tau} \mathcal{E}_{s}^{M}\right)$ est de dimension 0 ou 1 sur $\kappa_{A}$.

(ii) Il est équivalent de demander que pour tout $s \in \underline{r} \cap \underline{r}^{-}=\left(r_{1}, \ldots, r_{k-1}\right)$ le quotient ${ }^{\tau} \mathcal{E}^{M} /\left(\overline{\mathcal{E}}_{s}^{M} \oplus^{\tau} \mathcal{E}_{s}^{M}\right)$ soit de dimension 1 sur $\kappa_{A}$ ou bien que soient vérifiées les conditions suivantes:

- pour tout $s \in \underline{r}$, l'homomorphisme $\overline{\mathcal{E}}_{s^{-}}^{M} / \overline{\mathcal{E}}_{s}^{M} \rightarrow \mathcal{E}_{s}^{\prime \prime M} / \mathcal{E}_{s^{-}}^{\prime \prime M}$ est partout bien défini et inversible sur $X \otimes \kappa_{A}$,

- pour tout $s \in \underline{r}, \mathcal{E}_{s}^{\prime M} / \mathcal{E}_{s}^{M}$ est de dimension 1 sur $\kappa_{A}$,

- pour tout $s \in \underline{r}^{-}, \mathcal{E}_{s}^{\prime \prime M}=\mathcal{E}_{s}^{\prime M}$,

lesquelles impliquent que le chtouca dégénéré induit par $M$ soit un pré-chtouca itéré de rang $r$ sur $\mathrm{Spec} A$ dont la restriction au-dessus de $\mathrm{Spec} \kappa_{A}$ soit de type $\underline{r}$.

Démonstration. Il suffit de remarquer d'une part que pour tout $s \in \underline{r} \cup \underline{r}^{-}$les quotients $\mathcal{E}_{s}^{\prime M} / \mathcal{E}_{s}^{M}$ et $\mathcal{E}_{s}^{\prime M} / \mathcal{E}_{s}^{\prime \prime M}$ qui se plongent respectivement dans $\mathcal{E}^{\prime M} / \mathcal{E}^{M}$ et $\mathcal{E}^{\prime M} / \mathcal{E}^{\prime \prime M}$ sont de dimension 0 ou 1 sur $\kappa_{A}$ et d'autre part que pour tout $s \in \underline{r}$ on a des plongements partout bien définis sur $X \otimes \kappa_{A}$ entre fibrés de même rang

$$
\begin{gathered}
\operatorname{det}\left({ }^{\tau} \mathcal{E}^{M} / \overline{\mathcal{E}}_{s^{-}}^{M}\right) \hookrightarrow \operatorname{det}\left(\mathcal{E}_{s^{-}}^{\prime \prime M}\right), \\
\operatorname{det}\left({ }^{\tau} \mathcal{E}^{M} / \overline{\mathcal{E}}_{s^{-}}^{M}\right) \otimes \overline{\mathcal{E}}_{s^{-}}^{M} / \overline{\mathcal{E}}_{s}^{M} \hookrightarrow \operatorname{det}\left(\mathcal{E}_{s^{-}}^{\prime \prime M}\right) \otimes \mathcal{E}_{s}^{\prime \prime M} / \mathcal{E}_{s^{-}}^{\prime \prime M} .
\end{gathered}
$$

On conclut par des considérations de degrés.

Rappelons que dans le lemme $5 \mathrm{du}$ paragraphe $2 \mathrm{a}$, on a fait correspondre de manière biunivoque à tout sous- $\varphi$-espace $\widehat{W}$ de $\widehat{V}$ compatible avec la filtration $\left(\widehat{V}_{s}(M)\right)_{s \in \underline{r}}$ canoniquement associée au $\varphi$-réseau itéré $M$ un bon sous-espace $\widehat{W}^{\widehat{M}}$ de $V^{M}=M / \pi_{A} M$.

Comme $V^{M}$ s'identifie aux fibres génériques des fibrés $\mathcal{E}^{M}, \mathcal{E}^{\prime M}$ et $\mathcal{E}^{\prime \prime M}$ sur $X \otimes \kappa_{A}$, on peut considérer les sous-fibrés maximaux $\mathcal{E}_{W}^{M}, \mathcal{E}_{\widehat{W}}^{\prime M}$ et $\mathcal{E}_{W}^{\prime \prime M}$ engendrés par $\widehat{W}^{\widehat{M}}$. Ils sont tels que pour un certain $s \in \underline{r}$, on ait $\mathcal{E}_{s^{-}}^{M} \varsubsetneqq \mathcal{E}_{\widehat{W}}^{M} \subseteq \mathcal{E}_{s}^{M}$, $\mathcal{E}_{s^{-}}^{\prime M} \varsubsetneqq \mathcal{E}_{\widehat{W}}^{\prime M} \subseteq \mathcal{E}_{s}^{\prime M}$ et $\mathcal{E}_{s^{-}}^{\prime \prime M} \varsubsetneqq \mathcal{E}_{\widehat{W}}^{\prime \prime M} \subseteq \mathcal{E}_{s}^{\prime \prime M}$. Les sous-fibrés de $\mathcal{E}^{M}, \mathcal{E}^{\prime M}$ et $\mathcal{E}^{\prime \prime M}$ qui s'obtiennent de cette façon seront appelés les bons sous-objets. 
Proposition 9. Soient $M$ et $M^{\prime}$ deux $\varphi$-réseaux itérés dans $V$.

Et soit $\left(\widehat{W}_{w}\right)_{w \in \underline{w}}$ une filtration croissante de $\widehat{V}$ qui raffine les deux filtrations canoniquement associées à $M$ et $M^{\prime}$.

Soient alors $\left(\mathcal{E}_{\widehat{W}_{w}}^{M}=\mathcal{E}_{w}^{M}\right)_{w \in \underline{w}}$ et $\left(\mathcal{E}_{\widehat{W}_{w}}^{M^{\prime}}=\mathcal{E}_{w}^{M^{\prime}}\right)_{w \in \underline{w}}$ les deux filtrations correspondantes de $\mathcal{E}^{M}$ et $\mathcal{E}^{M^{\prime}}$ par des bons sous-objets.

(i) Si $\operatorname{deg} \mathcal{E}_{w}^{M}=\operatorname{deg} \mathcal{E}_{w}^{M^{\prime}}, \forall w \in \underline{w}$, on a des isomorphismes canoniques $\mathcal{E}_{w}^{M} / \mathcal{E}_{w^{-}}^{M}$ $\cong \mathcal{E}_{w}^{M^{\prime}} / \mathcal{E}_{w^{-}}^{M^{\prime}}, w \in \underline{w}$.

(ii) S'il existe $w_{0} \in \underline{w} \cap \underline{w}^{-}$tel que $\operatorname{deg} \mathcal{E}_{w_{0}}^{M}>\operatorname{deg} \mathcal{E}_{w_{0}}^{M^{\prime}}$ et $\operatorname{deg} \mathcal{E}_{w}^{M}=\operatorname{deg} \mathcal{E}_{w}^{M^{\prime}}$, $\forall w \in \underline{w}, w \neq w_{0}$, on a des isomorphismes canoniques

$$
\mathcal{E}_{w}^{M} / \mathcal{E}_{w^{-}}^{M} \cong \mathcal{E}_{w}^{M^{\prime}} / \mathcal{E}_{w^{-}}^{M^{\prime}}, w \in \underline{w}, w \neq w_{0}, w_{0}^{+},
$$

et des plongements canoniques

$$
\mathcal{E}_{w_{0}}^{M^{\prime}} / \mathcal{E}_{w_{0}^{-}}^{M^{\prime}} \hookrightarrow \mathcal{E}_{w_{0}}^{M} / \mathcal{E}_{w_{0}^{-}}^{M}, \mathcal{E}_{w_{0}^{+}}^{M} / \mathcal{E}_{w_{0}}^{M} \hookrightarrow \mathcal{E}_{w_{0}^{+}}^{M^{\prime}} / \mathcal{E}_{w_{0}}^{M^{\prime}} .
$$

Démonstration. Pour tout $d \in \mathbb{Z}, M^{\prime \prime}=M+\pi_{A}^{d} M^{\prime}$ est un réseau dans $V$. La fibre au-dessus de $X \otimes \kappa_{A}$ du fibré $\mathcal{E}\left(M^{\prime \prime}\right)$ localement libre sur $X \otimes A$ induit par $M^{\prime \prime}$ est un fibré localement libre $\mathcal{E}^{M^{\prime \prime}}$. La filtration $\left(\widehat{W}_{w}\right)_{w \in \underline{w}}$ de $\widehat{V}$ induit sur lui une filtration croissante $\left(\mathcal{E}_{w}^{M^{\prime \prime}}\right)_{w \in \underline{w}}$ par des sous-fibrés maximaux.

Les inclusions $M \subseteq M^{\prime \prime}, \pi_{A}^{d} M^{\prime} \subseteq M^{\prime \prime}$ induisent pour tout $w \in \underline{w}$ deux homomorphismes entre fibrés de même rang

$$
\mathcal{E}_{w}^{M} / \mathcal{E}_{w^{-}}^{M} \longrightarrow \mathcal{E}_{w}^{M^{\prime \prime}} / \mathcal{E}_{w^{-}}^{M^{\prime \prime}}, \mathcal{E}_{w}^{M^{\prime}} / \mathcal{E}_{w^{-}}^{M^{\prime}} \longrightarrow \mathcal{E}_{w}^{M^{\prime \prime}} / \mathcal{E}_{w^{-}}^{M^{\prime \prime}}
$$

dont l'un au moins est un plongement. Et pour tout $w_{1} \in \underline{w}$, il existe une unique façon d'avoir choisi $d$ - qu'on dit alors adapté à $w_{1}$ - de telle sorte que les deux homomorphismes correspondants soient des plongements.

Dans le cas (i), on voit donc que pour tout $w$

$$
\operatorname{deg} \mathcal{E}_{w}^{M^{\prime \prime}} / \mathcal{E}_{w^{-}}^{M^{\prime \prime}} \geq \operatorname{deg} \mathcal{E}_{w}^{M} / \mathcal{E}_{w^{-}}^{M}=\operatorname{deg} \mathcal{E}_{w}^{M^{\prime}} / \mathcal{E}_{w^{-}}^{M^{\prime}}
$$

et en fait il y a nécessairement égalité puisque $\operatorname{deg} \mathcal{E}^{M^{\prime \prime}}=\operatorname{deg} \mathcal{E}^{M}=\operatorname{deg} \mathcal{E}^{M^{\prime}}$.

Lorsque $d$ est adapté à $w_{1}$, les plongements

$$
\mathcal{E}_{w_{1}}^{M} / \mathcal{E}_{w_{1}^{-}}^{M} \hookrightarrow \mathcal{E}_{w_{1}}^{M^{\prime \prime}} / \mathcal{E}_{w_{1}^{-}}^{M^{\prime \prime}}, \mathcal{E}_{w_{1}}^{M^{\prime}} / \mathcal{E}_{w_{1}^{-}}^{M^{\prime}} \hookrightarrow \mathcal{E}_{w_{1}}^{M^{\prime \prime}} / \mathcal{E}_{w_{1}^{-}}^{M^{\prime \prime}}
$$

qui sont entre fibrés de même rang et même degré sont nécessairement des isomorphismes. On conclut en choisissant $d$ successivement adapté à chaque $w_{1} \in \underline{w}$.

Dans le cas (ii), on a

$$
\begin{aligned}
& \operatorname{deg} \mathcal{E}_{w}^{M^{\prime \prime}} / \mathcal{E}_{w^{-}}^{M^{\prime \prime}} \geq \operatorname{deg} \mathcal{E}_{w}^{M} / \mathcal{E}_{w^{-}}^{M}=\operatorname{deg} \mathcal{E}_{w}^{M^{\prime}} / \mathcal{E}_{w^{-}}^{M^{\prime}}, \forall w \neq w_{0}, w_{0}^{+}, \\
& \operatorname{deg} \mathcal{E}_{w_{0}}^{M^{\prime \prime}} / \mathcal{E}_{w_{0}^{-}}^{M^{\prime \prime}} \geq \operatorname{deg} \mathcal{E}_{w_{0}}^{M^{\prime}} / \mathcal{E}_{w_{0}^{-}}^{M^{\prime}}, \\
& \operatorname{deg} \mathcal{E}_{w_{0}^{+}}^{M^{\prime \prime}} / \mathcal{E}_{w_{0}}^{M^{\prime \prime}} \geq \operatorname{deg} \mathcal{E}_{w_{0}^{+}}^{M} / \mathcal{E}_{w_{0}}^{M} .
\end{aligned}
$$

Lorsque $d$ est choisi adapté à $w_{1}=w_{0}$, on a aussi $\operatorname{deg} \mathcal{E}_{w_{0}}^{M^{\prime \prime}} / \mathcal{E}_{w_{0}^{-}}^{M^{\prime \prime}} \geq \operatorname{deg} \mathcal{E}_{w_{0}}^{M} / \mathcal{E}_{w_{0}^{-}}^{M}$ donc $\mathcal{E}_{w_{0}}^{M} / \mathcal{E}_{w_{0}^{-}}^{M} \hookrightarrow \mathcal{E}_{w_{0}}^{M^{\prime \prime}} / \mathcal{E}_{w_{0}^{-}}^{M^{\prime \prime}}$ est un isomorphisme ainsi que $\mathcal{E}_{w_{0}^{+}}^{M} / \mathcal{E}_{w_{0}}^{M} \hookrightarrow \mathcal{E}_{w_{0}^{+}}^{M^{\prime \prime}} / \mathcal{E}_{w_{0}}^{M^{\prime \prime}}$.

Et lorsque $d$ est choisi adapté à $w_{1}=w_{0}^{+}$, on a aussi $\operatorname{deg} \mathcal{E}_{w_{0}^{+}}^{M^{\prime \prime}} / \mathcal{E}_{w_{0}}^{M^{\prime \prime}} \geq \operatorname{deg} \mathcal{E}_{w_{0}^{+}}^{M^{\prime}} / \mathcal{E}_{w_{0}}^{M^{\prime}}$ donc $\mathcal{E}_{w_{0}^{+}}^{M^{\prime}} / \mathcal{E}_{w_{0}}^{M^{\prime}} \hookrightarrow \mathcal{E}_{w_{0}^{+}}^{M^{\prime \prime}} / \mathcal{E}_{w_{0}}^{M^{\prime \prime}}$ est un isomorphisme ainsi que $\mathcal{E}_{w_{0}}^{M^{\prime}} / \mathcal{E}_{w_{0}^{-}}^{M^{\prime}} \hookrightarrow \mathcal{E}_{w_{0}}^{M^{\prime \prime}} / \mathcal{E}_{w_{0}^{-}}^{M^{\prime \prime}}$. 
Il résulte enfin de ce qu'on vient de voir dans ces deux cas que lorsque $d$ est choisi adapté à $w_{1} \neq w_{0}, w_{0}^{+}$, on a toujours ou bien $\operatorname{deg} \mathcal{E}_{w_{0}}^{M^{\prime \prime}} / \mathcal{E}_{w_{0}^{-}}^{M^{\prime \prime}} \geq \operatorname{deg} \mathcal{E}_{w_{0}}^{M} / \mathcal{E}_{w_{0}^{-}}^{M}>$ $\operatorname{deg} \mathcal{E}_{w_{0}}^{M^{\prime}} / \mathcal{E}_{w_{0}^{-}}^{M^{\prime}}$ ou bien $\operatorname{deg} \mathcal{E}_{w_{0}^{+}}^{M^{\prime \prime}} / \mathcal{E}_{w_{0}}^{M^{\prime \prime}} \geq \operatorname{deg} \mathcal{E}_{w_{0}^{+}}^{M^{\prime}} / \mathcal{E}_{w_{0}}^{M^{\prime}}>\operatorname{deg} \mathcal{E}_{w_{0}^{+}}^{M} / \mathcal{E}_{w_{0}}^{M}$.

On conclut alors comme dans la partie (i).

c) Transformations des chtoucas dégénérés. Dans ce paragraphe, nous allons introduire certaines opérations de transformations des $\varphi$-réseaux itérés en regardant leurs effets sur les chtoucas dégénérés associés, opérations que nous emploierons dans la construction finale au paragraphe $2 \mathrm{e}$.

Commençons par citer le lemme suivant:

Lemme 10 (Langton). Soient $A$ un anneau de valuation discrète contenant $\mathbb{F}_{q}, \mathcal{E}$ un fibré localement libre sur $X \otimes K_{A}, V$ sa fibre générique, $M$ un réseau dans $V$, $W$ un sous-espace vectoriel de $V^{M}=M / \pi_{A} M$ et $M^{\prime}$ le réseau $\operatorname{Ker}\left[M \rightarrow V^{M} / W\right]$.

Soient $\mathcal{E}(M)$ et $\mathcal{E}\left(M^{\prime}\right)$ les fibré localement libres sur $X \otimes A$ induits par $M$ et $M^{\prime}, \mathcal{E}^{M}$ et $\mathcal{E}^{M^{\prime}}$ leurs restrictions au-dessus de $X \otimes \kappa_{A}, \mathcal{E}_{W}^{M}$ et $\mathcal{E}_{W^{\prime}}^{M^{\prime}}$ les sous-fibrés maximaux de $\mathcal{E}^{M}$ et $\mathcal{E}^{M^{\prime}}$ engendrés par $W$ et $W^{\prime}=\operatorname{Ker}\left[M^{\prime} / \pi_{A} M^{\prime} \rightarrow W\right]$.

Alors on a les suites exactes canoniques

$$
\begin{aligned}
& 0 \longrightarrow \mathcal{E}_{W^{\prime}}^{M^{\prime}} \longrightarrow \mathcal{E}^{M^{\prime}} \longrightarrow \mathcal{E}_{W}^{M} \longrightarrow 0, \\
& 0 \longrightarrow \mathcal{E}_{W}^{M} \longrightarrow \mathcal{E}^{M} \longrightarrow \mathcal{E}_{W^{\prime}}^{M^{\prime}} \longrightarrow 0
\end{aligned}
$$

Comme dans le paragraphe précédent, on fixe un chtouca $\left(\begin{array}{c}\mathcal{E} \hookrightarrow \mathcal{E}^{\prime} \\ \mathcal{E}=\mathcal{E}^{\prime \prime} \nearrow\end{array}\right)$ de rang $r \geq 1$ au-dessus du point générique Spec $K_{A}$ d'un anneau de valuation discrète $A$ contenant $\mathbb{F}_{q}$. La fibre générique est un $\varphi$-espace $V$ de dimension $r$ sur $K_{A_{X}}$.

Du lemme 10 on déduit:

Proposition 11. Soient $M$ un $\varphi$-réseau itéré dans $V,\left(\widehat{W}_{w}\right)_{w \in \underline{w}}$ une filtration de $\widehat{V}$ par des sous- $\varphi$-espaces qui raffine la filtration canoniquement associée à $M$ et $w_{0}$ un élément de $\underline{w} \cap \underline{w}^{-}$.

(i) Soit $M^{1}$ le $\varphi$-réseau itéré transformé de $M$ par $\widehat{W}_{w_{0}}$ (qui donc figure dans la filtration de $\widehat{V}$ canoniquement associée à $M^{1}$ ). Alors, en notant $\mathcal{E}_{w_{0}}^{M}=\mathcal{E}_{\widehat{W}_{w_{0}}}^{M}$ le bon sous-objet de $\mathcal{E}^{M}$ induit par $\widehat{W}_{w_{0}}$, on a les suites exactes canoniques

$$
\begin{aligned}
& 0 \longrightarrow \overline{\mathcal{E}}_{w_{0}}^{M^{1}} \longrightarrow{ }^{\tau} \mathcal{E}^{M^{1}} \longrightarrow{ }^{\tau} \mathcal{E}_{w_{0}}^{M} \longrightarrow 0 \\
& 0 \longrightarrow{ }^{\tau} \mathcal{E}_{w_{0}}^{M} \longrightarrow{ }^{\tau} \mathcal{E}^{M} \longrightarrow \overline{\mathcal{E}}_{w_{0}}^{M^{1}} \longrightarrow 0
\end{aligned}
$$

De plus, on a un plongement canonique $\mathcal{E}_{w_{0}}^{M^{1}} \hookrightarrow \mathcal{E}_{w_{0}}^{M}$ et le quotient $\mathcal{E}_{w_{0}}^{M} / \mathcal{E}_{w_{0}}^{M^{1}}$ est de dimension 0 ou 1 sur $\kappa_{A}$.

(ii) Plus généralement, soit $M, M^{1}, M^{2}, \ldots, M^{m}=M^{\prime}$ une suite finie de transformés successifs de $M$ par $\widehat{W}_{w_{0}}$, telle que $\mathcal{E}_{w_{0}}^{M}=\mathcal{E}_{w_{0}}^{M^{1}}=\cdots=\mathcal{E}_{w_{0}}^{M^{m-1}}$. Alors on a des suites exactes canoniques

$$
\begin{gathered}
0 \longrightarrow \overline{\mathcal{E}}_{w_{0}}^{M^{\prime}} \longrightarrow{ }^{\tau} \mathcal{E}^{M^{\prime}} \longrightarrow{ }^{\tau} \mathcal{E}_{w_{0}}^{M} \longrightarrow 0 \\
0 \longrightarrow{ }^{\tau} \mathcal{E}_{w_{0}}^{M} \longrightarrow{ }^{\tau} \mathcal{E}^{M} \longrightarrow \overline{\mathcal{E}}_{w_{0}}^{M^{\prime}} \longrightarrow 0
\end{gathered}
$$

ainsi qu'un plongement canonique $\mathcal{E}_{w_{0}}^{M^{\prime}} \hookrightarrow \mathcal{E}_{w_{0}}^{M}$ dont le conoyau $\mathcal{E}_{w_{0}}^{M} / \mathcal{E}_{w_{0}}^{M^{\prime}}$ est de dimension 0 ou 1 sur $\kappa_{A}$. 
Démonstration. (i) Ces deux suites exactes ne sont autres que celles du lemme 10 dans le cas particulier que nous envisageons. De même, le plongement $\mathcal{E}_{w_{0}}^{M^{1}} \hookrightarrow \mathcal{E}_{w_{0}}^{M}$ est induit par la première suite exacte du lemme 10. Enfin, le quotient ${ }^{\tau} \mathcal{E}_{w_{0}}^{M} /{ }^{\tau} \mathcal{E}_{w_{0}}^{M^{1}}$ s'identifie à ${ }^{\tau} \mathcal{E}^{M^{1}} /\left(\overline{\mathcal{E}}_{w_{0}}^{M^{1}} \oplus{ }^{\tau} \mathcal{E}_{w_{0}}^{M^{1}}\right)$; il est de dimension 0 ou 1 sur $\kappa_{A}$ d'après la proposition 8 (i) du paragraphe $2 \mathrm{~b}$.

(ii) En effet, on a d'après (i) les deux suites exactes

$$
\begin{gathered}
0 \longrightarrow \overline{\mathcal{E}}_{w_{0}}^{M^{\prime}} \longrightarrow{ }^{\tau} \mathcal{E}^{M^{\prime}} \longrightarrow{ }^{\tau} \mathcal{E}_{w_{0}}^{M^{m-1}} \longrightarrow 0 \\
0 \longrightarrow{ }^{\tau} \mathcal{E}_{w_{0}}^{M} \longrightarrow{ }^{\tau} \mathcal{E}^{M} \longrightarrow \overline{\mathcal{E}}_{w_{0}}^{M^{1}} \longrightarrow 0
\end{gathered}
$$

et des isomorphismes canoniques

$$
\begin{gathered}
\mathcal{E}_{w_{0}}^{M^{m-1}}=\mathcal{E}_{w_{0}}^{M^{m-2}}=\cdots=\mathcal{E}_{w_{0}}^{M^{1}}=\mathcal{E}_{w_{0}}^{M}, \\
\overline{\mathcal{E}}_{w_{0}}^{M^{1}}={ }^{\tau} \mathcal{E}^{M^{1}} /{ }^{\tau} \mathcal{E}_{w_{0}}^{M^{1}}=\overline{\mathcal{E}}_{w_{0}}^{M^{2}}=\cdots={ }^{\tau} \mathcal{E}^{M^{m-1}} /{ }^{\tau} \mathcal{E}_{w_{0}}^{M^{m-1}}=\overline{\mathcal{E}}_{w_{0}}^{M^{\prime}} .
\end{gathered}
$$

Quant à la seconde assertion, elle se déduit immédiatement de celle de la partie (i).

Dans la situation de la proposition 11 (ii), on dira que $M^{\prime}$ est le transformé strict de $M$ par $\widehat{W}_{w_{0}}$ quand $\mathcal{E}_{w_{0}}^{M} / \mathcal{E}_{w_{0}}^{M^{\prime}}$ est de dimension 1 ou - ce qui revient au même - quand ${ }^{\tau} \mathcal{E}^{M^{\prime}} /\left(\overline{\mathcal{E}}_{w_{0}}^{M^{\prime}} \oplus{ }^{\tau} \mathcal{E}_{w_{0}}^{M^{\prime}}\right)$ est de dimension 1. Et $M$ sera alors appelé un transformé réciproque strict de $M^{\prime}$ par $\widehat{W}_{w_{0}}$.

Toujours dans cette situation, on dira que $M$ est un modifié de $M^{\prime}$ par $\widehat{W}_{w_{0}}$ lorsque le plongement $\mathcal{E}_{w_{0}}^{M^{\prime}} \hookrightarrow \mathcal{E}_{w_{0}}^{M}$ est un isomorphisme. Et $M$ sera appelé le modifié maximal de $M^{\prime}$ par $\widehat{W}_{w_{0}}$ (il en existe au plus un) si de plus

- ou bien $\widehat{W}_{w_{0}}$ ne figure pas dans la filtration de $\widehat{V}$ canoniquement associée au $\varphi$-réseau itéré $M$,

- ou bien $\widehat{W}_{w_{0}}$ figure dans cette filtration et le quotient ${ }^{\tau} \mathcal{E}^{M} /\left(\overline{\mathcal{E}}_{w_{0}}^{M} \oplus^{\tau} \mathcal{E}_{w_{0}}^{M}\right)$ est de dimension 1 (ce qui revient à dire, au cas où $M$ admettrait un transformé réciproque par $\widehat{W}_{w_{0}}$, que $M$ serait le transformé strict de celui-ci).

Enfin, étant donnés $M$ un $\varphi$-réseau itéré dans $V$, $\left(\widehat{W}_{w}\right)_{w \in \underline{w}}$ une filtration de $\widehat{V}$ raffinant celle associée à $M$ et $w_{0}$ un élément de $\underline{w} \cap \underline{w}^{-}$, on dira qu'un $\varphi$-réseau itéré $M^{\prime}$ est le modifié maximal de $M$ par les $\widehat{W}_{w}, w \geq w_{0}$, s'il existe un élément $w_{1} \geq w_{0}$ dans $\underline{w}$ et une suite $\left(M^{w}\right)_{w_{0} \leq w \leq w_{1}}$ de $\varphi$-réseaux itérés tels que:

- On a $M^{w_{0}}=M$ et pour tout $w, w_{0} \leq w<w_{1}, M^{w_{+}}$est le modifié maximal de $M^{w}$ par $\widehat{W}_{w}$ et $\widehat{W}_{w}$ ne figure pas dans la filtration de $\widehat{V}$ associée à $M^{w_{+}}$.

- Ou bien $w_{1}=r$, ou bien $w_{1}<r$ et $M^{\prime}$ est le modifié maximal de $M^{w_{1}}$ par $\widehat{W}_{w_{1}}$, $\widehat{W}_{w_{1}}$ figure dans la filtration de $\widehat{V}$ associée à $M^{\prime}$ et le quotient ${ }^{\tau} \mathcal{E}^{M^{\prime}} /\left(\overline{\mathcal{E}}_{w_{1}}^{M^{\prime}} \oplus^{\tau} \mathcal{E}_{w_{1}}^{M^{\prime}}\right)$ est de dimension 1.

Nous allons déduire de la proposition 11:

Proposition 12. Soient $M$ un $\varphi$-réseau itéré dans $V$ et $\left(\widehat{W}_{w}\right)_{w \in \underline{w}}$ une filtration de $\widehat{V}$ par des sous- $\varphi$-espaces qui est la réunion de la filtration canoniquement associée $\grave{a} M$ et d'un élément $\widehat{W}_{w_{0}}, w_{0} \in \underline{w} \cap \underline{w}^{-}$.

On suppose que pour tout $w>w_{0}$ dans $\underline{w}^{-}$, on a $\overline{\mathcal{E}}_{w}^{M} \oplus^{\tau} \mathcal{E}_{w}^{M} \mp^{\tau} \mathcal{E}^{M}$ et $\overline{\mathcal{E}}_{w}^{M}+$ ${ }^{\tau} \mathcal{E}_{w^{+}}^{M}={ }^{\tau} \mathcal{E}^{M}$.

On suppose d'autre part que $M$ admet un transformé strict $M^{1}$ par $\widehat{W}_{w_{0}}$. 
Si le conoyau du plongement canonique $\mathcal{E}^{M} / \mathcal{E}_{w_{0}^{+}}^{M} \hookrightarrow \mathcal{E}^{M^{1}} / \mathcal{E}_{w_{0}^{+}}^{M^{1}}$ est nul, on pose $M^{2}=M^{1}$.

Si au contraire ce conoyau est de dimension 1 sur $\kappa_{A}$, on suppose que $M^{1}$ admet un transformé réciproque $M^{2}$ par $\widehat{W}_{w_{0}^{+}}$.

Enfin, on suppose que $M^{2}$ admet un modifié maximal $M^{3}=M^{\prime}$ par les $\widehat{W}_{w}$, $w \geq w_{0}^{+}$.

Alors le chtouca dégénéré induit par $M^{\prime}$ vérifie:

(i) Pour tout $w \geq w_{0}^{+}$dans $\underline{w}^{-}$, on a l'égalité $\operatorname{deg} \mathcal{E}_{w}^{M^{\prime}}=\operatorname{deg} \mathcal{E}_{w}^{M}$ et un isomorphisme canonique $\mathcal{E}_{w^{+}}^{M^{\prime}} / \mathcal{E}_{w}^{M^{\prime}} \cong \mathcal{E}_{w^{+}}^{M} / \mathcal{E}_{w}^{M}$.

(ii) Pour tout $w \geq w_{0}^{+}$dans $\underline{w}^{-}$tel que $\widehat{W}_{w}$ figure dans la filtration de $\widehat{V}$ associée $\grave{a} M^{\prime}$, on a des isomorphismes canoniques

$$
\mathcal{E}^{M^{\prime}} / \mathcal{E}_{w}^{M^{\prime}} \cong \mathcal{E}^{M} / \mathcal{E}_{w}^{M}, \quad \overline{\mathcal{E}}_{w}^{M^{\prime}} \cong \overline{\mathcal{E}}_{w}^{M},
$$

avec donc

$$
\overline{\mathcal{E}}_{w}^{M^{\prime}} \oplus{ }^{\tau} \mathcal{E}_{w}^{M^{\prime}} \varsubsetneqq{ }^{\tau} \mathcal{E}^{M^{\prime}}, \quad \overline{\mathcal{E}}_{w}^{M^{\prime}}+{ }^{\tau} \mathcal{E}_{w^{+}}^{M^{\prime}}={ }^{\tau} \mathcal{E}^{M^{\prime}}
$$

(iii) On dispose de plongements canoniques

$$
\mathcal{E}_{w_{0}^{+}}^{M} / \mathcal{E}_{w_{0}}^{M} \hookrightarrow \mathcal{E}_{w_{0}^{+}}^{M^{\prime}} / \mathcal{E}_{w_{0}}^{M^{\prime}} \quad \text { et } \quad \mathcal{E}_{w_{0}}^{M^{\prime}} \hookrightarrow \mathcal{E}_{w_{0}}^{M}
$$

dont les conoyaux sont de même dimension 0 ou 1.

Lorsque ces conoyaux sont de dimension 1 , soit $\operatorname{deg} \mathcal{E}_{w_{0}}^{M^{\prime}}=\operatorname{deg} \mathcal{E}_{w_{0}}^{M}-1$, on a de plus $\overline{\mathcal{E}}_{w_{0}}^{M^{\prime}} \oplus{ }^{\tau} \mathcal{E}_{w_{0}}^{M^{\prime}} \varsubsetneqq \tau \mathcal{E}^{M^{\prime}}, \overline{\mathcal{E}}_{w_{0}}^{M^{\prime}}+{ }^{\tau} \mathcal{E}_{w_{0}^{+}}^{M^{\prime}}={ }^{\tau} \mathcal{E}^{M^{\prime}}$.

Démonstration. Montrons d'abord qu'il y a un isomorphisme canonique $\mathcal{E}^{M} / \mathcal{E}_{w_{0}^{+}}^{M} \cong$ $\mathcal{E}^{M^{2}} / \mathcal{E}_{w_{0}^{+}}^{M^{2}}$

C'est évident si le plongement $\mathcal{E}^{M} / \mathcal{E}_{w_{0}^{+}}^{M} \hookrightarrow \mathcal{E}^{M^{1}} / \mathcal{E}_{w_{0}^{+}}^{M^{1}}$ est un isomorphisme puisqu'alors on a posé $M^{2}=M^{1}$.

Dans le cas contraire, il suffit de prouver que le plongement

$$
{ }^{\tau} \mathcal{E}^{M^{2}} /{ }^{\tau} \mathcal{E}_{w_{0}^{+}}^{M^{2}} \cong \overline{\mathcal{E}}_{w_{0}^{+}}^{M^{1}} \hookrightarrow \overline{\mathcal{E}}_{w_{0}}^{M^{1}} / \overline{\mathcal{E}}_{w_{0}}^{M^{1}} \cap{ }^{\tau} \mathcal{E}_{w_{0}^{+}}^{M^{1}} \cong \tau{ }^{\tau} \mathcal{E}^{M} /{ }^{\tau} \mathcal{E}_{w_{0}^{+}}^{M}
$$

est un isomorphisme. Or on a

$$
\overline{\mathcal{E}}_{w_{0}^{+}}^{M^{1}} \oplus{ }^{\tau} \mathcal{E}_{w_{0}^{+}}^{M^{1}} \subseteq \overline{\mathcal{E}}_{w_{0}}^{M^{1}}+{ }^{\tau} \mathcal{E}_{w_{0}^{+}}^{M^{1}} \varsubsetneqq{ }^{\tau} \mathcal{E}^{M^{1}}
$$

donc la première inclusion est une égalité et on obtient $\overline{\mathcal{E}}_{w_{0}^{+}}^{M^{1}} \cong \overline{\mathcal{E}}_{w_{0}}^{M^{1}} / \overline{\mathcal{E}}_{w_{0}}^{M^{1}} \cap{ }^{\tau} \mathcal{E}_{w_{0}^{+}}^{M^{1}}$, puisque le quotient ${ }^{\tau} \mathcal{E}^{M^{1}} /\left(\overline{\mathcal{E}}_{w_{0}^{+}}^{M^{1}} \oplus{ }^{\tau} \mathcal{E}_{w_{0}^{+}}^{M^{1}}\right)$ est de dimension 1 au plus.

D'autre part, on dispose du plongement composé

$$
\mathcal{E}_{w_{0}^{+}}^{M} / \mathcal{E}_{w_{0}}^{M} \hookrightarrow \mathcal{E}_{w_{0}^{+}}^{M^{1}} / \mathcal{E}_{w_{0}}^{M^{1}} \hookrightarrow \mathcal{E}_{w_{0}^{+}}^{M^{2}} / \mathcal{E}_{w_{0}}^{M^{2}}
$$

ainsi que du plongement

$$
\mathcal{E}_{w_{0}}^{M^{2}} \hookrightarrow \mathcal{E}_{w_{0}}^{M}
$$

induit par l'inclusion $M^{2} \subseteq M$.

Comparons maintenant les chtoucas dégénérés induits par $M^{2}$ et par sa modification maximale $M^{\prime}=M^{3}$ par les $\widehat{W}_{w}, w \geq w_{0}^{+}$. Par construction, il existe $w_{1} \geq w_{0}^{+}$dans $\underline{w}$ tel que, pour $w_{0}^{+} \leq w<w_{1}, \widehat{W}_{w}$ ne figure pas dans la filtration 
de $\widehat{V}$ associée à $M^{\prime}$ et que, pour $w \geq w_{1}, \widehat{W}_{w}$ y figure. Et on a des isomorphismes canoniques

$$
\begin{aligned}
\mathcal{E}_{w_{0}^{+}}^{M^{2}} & \cong \mathcal{E}_{w_{0}^{+}}^{M^{\prime}}, \\
\mathcal{E}_{w^{+}}^{M^{2}} / \mathcal{E}_{w}^{M^{2}} & \cong \mathcal{E}_{w^{+}}^{M^{\prime}} / \mathcal{E}_{w}^{M^{\prime}}, \quad w_{0}^{+} \leq w<w_{1}, \\
\mathcal{E}^{M^{2}} / \mathcal{E}_{w_{1}}^{M^{2}} & \cong \mathcal{E}^{M^{\prime}} / \mathcal{E}_{w_{1}}^{M^{\prime}} .
\end{aligned}
$$

Ainsi (i) est démontré. D'autre part (ii) est vide si $w_{1}=r$. Sinon, par définition de la modification maximale $M^{\prime}$ de $M^{2}$, le conoyau de

$$
\overline{\mathcal{E}}_{w_{1}}^{M^{\prime}} \hookrightarrow{ }^{\tau} \mathcal{E}^{M^{\prime}} /{ }^{\tau} \mathcal{E}_{w_{1}}^{M^{\prime}} \cong{ }^{\tau} \mathcal{E}^{M} /{ }^{\tau} \mathcal{E}_{w_{1}}^{M}
$$

est de dimension 1 sur $\kappa_{A}$.

D'où un isomorphisme

$$
\overline{\mathcal{E}}_{w_{1}}^{M^{\prime}} \cong \overline{\mathcal{E}}_{w_{1}}^{M}
$$

et a fortiori, pour tout $w \geq w_{1}$ dans $\underline{w}^{-}$,

$$
\overline{\mathcal{E}}_{w}^{M^{\prime}} \cong \overline{\mathcal{E}}_{w}^{M}, \overline{\mathcal{E}}_{w}^{M^{\prime}} \oplus{ }^{\tau} \mathcal{E}_{w}^{M^{\prime}} \varsubsetneqq{ }^{\tau} \mathcal{E}^{M^{\prime}}, \overline{\mathcal{E}}_{w}^{M^{\prime}}+{ }^{\tau} \mathcal{E}_{w^{+}}^{M^{\prime}}={ }^{\tau} \mathcal{E}^{M^{\prime}},
$$

ce qui démontre (ii).

Par ailleurs, le conoyau du plongement $\mathcal{E}_{w_{0}}^{M^{1}} \hookrightarrow \mathcal{E}_{w_{0}}^{M}$ est de dimension 1 et celui du plongement $\mathcal{E}_{w_{0}}^{M^{1}} \hookrightarrow \mathcal{E}_{w_{0}}^{M^{2}}=\mathcal{E}_{w_{0}}^{M^{\prime}}$ est de dimension 0 ou 1 , ce qui achève de prouver la première assertion de (iii).

Voyons enfin ce qu'on peut déduire de l'hypothèse $\operatorname{deg} \mathcal{E}_{w_{0}}^{M^{\prime}}=\operatorname{deg} \mathcal{E}_{w_{0}}^{M}-1$ équivalente à $\mathcal{E}_{w_{0}}^{M^{1}}=\mathcal{E}_{w_{0}}^{M^{2}}=\mathcal{E}_{w_{0}}^{M^{\prime}}$.

D'après la relation $\overline{\mathcal{E}}_{w_{0}}^{M^{1}} \oplus{ }^{\tau} \mathcal{E}_{w_{0}}^{M^{1}} \varsubsetneqq^{\tau} \mathcal{E}^{M^{1}}$ et le plongement

$$
{ }^{\tau} \mathcal{E}^{M^{1}} / \overline{\mathcal{E}}_{w_{0}}^{M^{1}} \hookrightarrow{ }^{\tau} \mathcal{E}^{M^{2}} / \overline{\mathcal{E}}_{w_{0}}^{M^{2}} \hookrightarrow{ }^{\tau} \mathcal{E}^{M^{\prime}} / \overline{\mathcal{E}}_{w_{0}}^{M^{\prime}},
$$

on a $\overline{\mathcal{E}}_{w_{0}}^{M^{\prime}} \oplus{ }^{\tau} \mathcal{E}_{w_{0}}^{M^{\prime}} \varsubsetneqq{ }^{\tau} \mathcal{E}^{M^{\prime}}$. Et on note au passage que les quotients non nuls $\tau \mathcal{E}^{M^{1}} /\left(\overline{\mathcal{E}}_{w_{0}}^{M^{1}} \oplus \tau \mathcal{E}_{w_{0}}^{M^{1}}\right)$ et ${ }^{\tau} \mathcal{E}^{M^{\prime}} /\left(\overline{\mathcal{E}}_{w_{0}}^{M^{\prime}} \oplus{ }^{\tau} \mathcal{E}_{w_{0}}^{M^{\prime}}\right)$ étant de même dimension 1 , on a ${ }^{\tau} \mathcal{E}^{M^{1}} / \overline{\mathcal{E}}_{w_{0}}^{M^{1}} \cong \tau \mathcal{E}^{M^{2}} / \overline{\mathcal{E}}_{w_{0}}^{M^{2}} \cong \tau \mathcal{E}^{M^{\prime}} / \overline{\mathcal{E}}_{w_{0}}^{M^{\prime}}$.

Afin de montrer la relation

$$
\overline{\mathcal{E}}_{w_{0}}^{M^{\prime}}+{ }^{\tau} \mathcal{E}_{w_{0}^{+}}^{M^{\prime}}={ }^{\tau} \mathcal{E}^{M^{\prime}}
$$

il suffit donc de montrer $\overline{\mathcal{E}}_{w_{0}}^{M^{2}}+{ }^{\tau} \mathcal{E}_{w_{0}}^{M^{2}}={ }^{\tau} \mathcal{E}^{M^{2}}$.

Dans le cas où $M^{2}=M^{1}$, cela résulte de ce qu'alors ${ }^{\tau} \mathcal{E}^{M^{1}} /{ }^{\tau} \mathcal{E}_{w_{0}^{+}}^{M^{1}} \cong{ }^{\tau} \mathcal{E}^{M} /{ }^{\tau} \mathcal{E}_{w_{0}^{+}}^{M}$ et $\overline{\mathcal{E}}_{w_{0}}^{M^{1}} \cong{ }^{\tau} \mathcal{E}^{M} /{ }^{\tau} \mathcal{E}_{w_{0}}^{M}$. Dans le cas contraire, il s'agit de vérifier que le plongement $\overline{\mathcal{E}}_{w_{0}}^{M^{2}} / \overline{\mathcal{E}}_{w_{0}}^{M^{2}} \cap{ }^{\tau} \mathcal{E}_{w_{0}^{+}}^{M^{2}} \hookrightarrow{ }^{\tau} \mathcal{E}^{M^{2}} / \mathcal{E}_{w_{0}^{+}}^{M^{2}}$ est un isomorphisme, ce qui résulte de l'égalité des degrés de ces deux fibrés, laquelle est conséquence de

$$
\operatorname{deg} \overline{\mathcal{E}}_{w_{0}}^{M^{1}}=\operatorname{deg} \overline{\mathcal{E}}_{w_{0}}^{M^{2}}, \overline{\mathcal{E}}_{w_{0}}^{M^{2}} \cap{ }^{\tau} \mathcal{E}_{w_{0}^{+}}^{M^{2}} \cong \overline{\mathcal{E}}_{w_{0}}^{M^{1}} / \overline{\mathcal{E}}_{w_{0}^{+}}^{M^{1}},{ }^{\tau} \mathcal{E}^{M^{2}} /{ }^{\tau} \mathcal{E}_{w_{0}^{+}}^{M^{2}} \cong \overline{\mathcal{E}}_{w_{0}^{+}}^{M^{1}} .
$$

Ceci termine la démonstration de (iii). 
Dans la situation de la proposition 12, on dira que $M^{\prime}$ est le transformé de $M$ par les $\widehat{W}_{w}, w \geq w_{0}$.

Plus généralement, étant donnés $M$ un $\varphi$-réseau itéré de $V,\left(\widehat{W}_{w}\right)_{w \in \underline{w}}$ une filtration de $\widehat{V}$ raffinant celle $\left(\widehat{V}_{s}(M)\right)_{s \in \underline{r}}$ associée à $M$ et $w_{0}$ un élément de $\underline{w} \cap \underline{w}^{-}$, on appellera transformé de $M$ par les $\widehat{W}_{w}, w \geq w_{0}$, le transformé de $M$ (s'il existe) par $\widehat{W}_{w_{0}}$ et les $\widehat{V}_{s}(M), s>w_{0}$.

Puis, s'il existe une suite $M, M^{1}, M^{2}, \ldots, M^{m}=M^{\prime}$ de transformés successifs de $M$ par les $\widehat{W}_{w}, w \geq w_{0}$, telle que

$$
\operatorname{deg} \mathcal{E}_{w_{0}}^{M}=\operatorname{deg} \mathcal{E}_{w_{0}}^{M^{1}}=\cdots=\operatorname{deg} \mathcal{E}_{w_{0}}^{M^{m-1}}=\operatorname{deg} \mathcal{E}_{w_{0}}^{M^{\prime}}+1,
$$

on dira que $M^{\prime}$ est le transformé strict de $M$ par les $\widehat{W}_{w}, w \geq w_{0}$.

Proposition 13. Soient $M$ un $\varphi$-réseau itéré dans $V,\left(\widehat{W}_{w}\right)_{w \in \underline{w}}$ la filtration $d e \widehat{V}$ canoniquement associée à $M$ et $w_{0}$ un élément de $\underline{w} \cap \underline{w}^{-}$.

On suppose que pour tout $w \geq w_{0}^{+}$dans $\underline{w}^{-}$, on a

$$
\overline{\mathcal{E}}_{w}^{M} \oplus{ }^{\tau} \mathcal{E}_{w}^{M} \varsubsetneqq{ }^{\tau} \mathcal{E}^{M} \quad \text { et } \quad \overline{\mathcal{E}}_{w}^{M}+{ }^{\tau} \mathcal{E}_{w^{+}}^{M}={ }^{\tau} \mathcal{E}^{M} .
$$

On suppose d'autre part que $M$ admet un modifié maximal $M^{1}$ par $\widehat{W}_{w_{0}}$ et que $M^{1}$ admet un transformé réciproque $M^{2}$ par $\widehat{W}_{w_{0}}$.

Si le conoyau du plongement canonique $\mathcal{E}^{M^{2}} / \mathcal{E}_{w_{0}^{+}}^{M^{2}} \hookrightarrow \mathcal{E}^{M} / \mathcal{E}_{w_{0}^{+}}^{M}$ est nul, on pose $M^{\prime}=M^{2}$.

Si au contraire ce conoyau est de dimension 1 sur $\kappa_{A}$, on note $M^{\prime}$ le transformé de $M^{2}$ par $\widehat{W}_{w_{0}^{+}}$.

Alors le chtouca dégénéré induit par $M^{\prime}$ vérifie:

(i) Pour tout $w \geq w_{0}^{+}$dans $\underline{w}^{-}$, on a des isomorphismes canoniques

$$
\mathcal{E}^{M^{\prime}} / \mathcal{E}_{w}^{M^{\prime}} \cong \mathcal{E}^{M} / \mathcal{E}_{w}^{M}, \quad \overline{\mathcal{E}}_{w}^{M^{\prime}} \cong \overline{\mathcal{E}}_{w}^{M}
$$

avec donc

$$
\overline{\mathcal{E}}_{w}^{M^{\prime}} \oplus{ }^{\tau} \mathcal{E}_{w}^{M^{\prime}} \varsubsetneqq{ }^{\tau} \mathcal{E}^{M^{\prime}}, \quad \overline{\mathcal{E}}_{w}^{M^{\prime}}+{ }^{\tau} \mathcal{E}_{w^{+}}^{M^{\prime}}={ }^{\tau} \mathcal{E}^{M^{\prime}} .
$$

(ii) On dispose de plongements canoniques

$$
\mathcal{E}_{w_{0}^{+}}^{M^{\prime}} / \mathcal{E}_{w_{0}}^{M^{\prime}} \hookrightarrow \mathcal{E}_{w_{0}^{+}}^{M} / \mathcal{E}_{w_{0}}^{M} \quad \text { et } \quad \mathcal{E}_{w_{0}}^{M} \hookrightarrow \mathcal{E}_{w_{0}}^{M^{\prime}}
$$

dont les conoyaux sont de même dimension 0 ou 1 .

Démonstration. On a des plongements canoniques $\mathcal{E}_{w_{0}}^{M}=\mathcal{E}_{w_{0}}^{M^{1}} \hookrightarrow \mathcal{E}_{w_{0}}^{M^{2}}, \mathcal{E}^{M^{2}} / \mathcal{E}_{w_{0}}^{M^{2}}$ $\hookrightarrow \mathcal{E}^{M^{1}} / \mathcal{E}_{w_{0}}^{M^{1}}=\mathcal{E}^{M} / \mathcal{E}_{w_{0}}^{M}$ et $\overline{\mathcal{E}}_{w_{0}}^{M^{2}} \hookrightarrow \overline{\mathcal{E}}_{w_{0}}^{M^{1}} \hookrightarrow \overline{\mathcal{E}}_{w_{0}}^{M}$.

Comme le quotient ${ }^{\tau} \mathcal{E}^{M} /\left(\overline{\mathcal{E}}_{w_{0}^{+}}^{M} \oplus{ }^{\tau} \mathcal{E}_{w_{0}^{+}}^{M}\right)$ est de dimension 1 et les quotients ${ }^{\tau} \mathcal{E}^{M^{1}} /\left(\overline{\mathcal{E}}_{w_{0}^{+}}^{M^{1}} \oplus{ }^{\tau} \mathcal{E}_{w_{0}^{+}}^{M^{1}}\right)$ et ${ }^{\tau} \mathcal{E}^{M^{2}} /\left(\overline{\mathcal{E}}_{w_{0}^{+}}^{M^{2}} \oplus{ }^{\tau} \mathcal{E}_{w_{0}^{+}}^{M^{2}}\right)$ sont de dimension 1 au plus, on voit que nécessairement $\overline{\mathcal{E}}_{w_{0}^{+}}^{M^{1}}=\overline{\mathcal{E}}_{w_{0}^{+}}^{M}$ et même $\overline{\mathcal{E}}_{w_{0}^{+}}^{M^{2}}=\overline{\mathcal{E}}_{w_{0}^{+}}^{M} \operatorname{si} \operatorname{deg} \mathcal{E}_{w_{0}^{+}}^{M^{2}}=\operatorname{deg} \mathcal{E}_{w_{0}^{+}}^{M}$.

Ceci démontre ce qu'on voulait dans le cas $M^{\prime}=M^{2}$.

Dans le cas contraire où $\operatorname{deg} \mathcal{E}_{w_{0}^{+}}^{M^{2}}=\operatorname{deg} \mathcal{E}_{w_{0}^{+}}^{M}+1$, l'inclusion $M^{1} \subset M^{\prime}$ induit des plongements $\mathcal{E}_{w_{0}}^{M}=\mathcal{E}_{w_{0}}^{M^{1}} \hookrightarrow \mathcal{E}_{w_{0}}^{M^{\prime}}, \mathcal{E}^{M} / \mathcal{E}_{w_{0}^{+}}^{M}=\mathcal{E}^{M^{1}} / \mathcal{E}_{w_{0}^{+}}^{M^{1}} \hookrightarrow \mathcal{E}^{M^{\prime}} / \mathcal{E}_{w_{0}^{+}}^{M^{\prime}}$ et $\overline{\mathcal{E}}_{w_{0}^{+}}^{M}=$ 
$\overline{\mathcal{E}}_{w_{0}^{+}}^{M^{1}} \hookrightarrow \overline{\mathcal{E}}_{w_{0}^{+}}^{M^{\prime}}$. Or on a $\operatorname{deg} \overline{\mathcal{E}}_{w_{0}^{+}}^{M^{\prime}}=\operatorname{deg}{ }^{\tau} \mathcal{E}^{M^{2}} /{ }^{\tau} \mathcal{E}_{w_{0}^{+}}^{M^{2}}=\operatorname{deg} \overline{\mathcal{E}}_{w_{0}^{+}}^{M}$ donc les plongements $\overline{\mathcal{E}}_{w_{0}^{+}}^{M} \hookrightarrow \overline{\mathcal{E}}_{w_{0}^{+}}^{M^{\prime}}$ et $\mathcal{E}^{M} / \mathcal{E}_{w_{0}^{+}}^{M} \hookrightarrow \mathcal{E}^{M^{\prime}} / \mathcal{E}_{w_{0}^{+}}^{M^{\prime}}$ sont des isomorphismes.

Enfin, on dispose du plongement composé

$$
\mathcal{E}_{w_{0}^{+}}^{M^{\prime}} / \mathcal{E}_{w_{0}}^{M^{\prime}} \hookrightarrow \mathcal{E}_{w_{0}^{+}}^{M^{2}} / \mathcal{E}_{w_{0}}^{M^{2}} \cong \mathcal{E}_{w_{0}^{+}}^{M^{1}} / \mathcal{E}_{w_{0}}^{M^{1}} \cong \mathcal{E}_{w_{0}^{+}}^{M} / \mathcal{E}_{w_{0}}^{M},
$$

ce qui termine la démonstration.

Dans la situation de la proposition 13, on dira que $M^{\prime}$ est le transformé réciproque de $M$ par les $\widehat{W}_{w}, w \geq w_{0}$.

Plus généralement, étant donnés $M$ un $\varphi$-réseau itéré de $V,\left(\widehat{W}_{w}\right)_{w \in \underline{w}}$ une filtration de $\widehat{V}$ raffinant celle $\left(\widehat{V}_{s}(M)\right)_{s \in \underline{r}}$ associée à $M$ et $w_{0}$ un élément de $\underline{w} \cap \underline{w}^{-} \cap \underline{r}$, on appellera transformé réciproque de $M$ par les $\widehat{W}_{w}, w \geq w_{0}$, le transformé réciproque de $M$ (s'il existe) par les $\widehat{V}_{s}(M), s \geq w_{0}$.

Puis, s'il existe une suite $M, M^{1}, M^{2}, \ldots, M^{m}=M^{\prime}$ de transformés réciproques successifs de $M$ par les $\widehat{W}_{w}, w \geq w_{0}$, telle que

$$
\operatorname{deg} \mathcal{E}_{w_{0}}^{M}=\operatorname{deg} \mathcal{E}_{w_{0}}^{M^{1}}=\cdots=\operatorname{deg} \mathcal{E}_{w_{0}}^{M^{m-1}}=\operatorname{deg} \mathcal{E}_{w_{0}}^{M^{\prime}}-1,
$$

on dira que $M^{\prime}$ est le transformé réciproque strict de $M$ par les $\widehat{W}_{w}, w \geq w_{0}$.

d) Critères d'existence de telles transformations. Comme dans les paragraphes précédents, on fixe un chtouca $\left(\begin{array}{ccc}\mathcal{E} & \hookrightarrow & \mathcal{E}^{\prime} \\ \tau \mathcal{E}=\mathcal{E}^{\prime \prime} & \nearrow\end{array}\right)$ de $\operatorname{rang} r \geq 1$ audessus du point générique Spec $K_{A}$ d'un anneau de valuation discrète $A$ contenant $\mathbb{F}_{q}$. Sa fibre générique est un $\varphi$-espace $V$ de dimension $r$ sur $K_{A_{X}}$.

Proposition 14. Soit $p:[0, r] \rightarrow \mathbb{R}_{+}$un polygone tel que le chtouca $\left(\begin{array}{cccc}\mathcal{E} & \hookrightarrow & \mathcal{E}^{\prime} \\ & \nearrow & \\ \tau & & \end{array}\right)$ au-dessus de $\operatorname{Spec} K_{A}$ soit dans l'ouvert $\mathrm{Cht}^{r, \bar{p} \leq p}$ de $\mathrm{Cht}^{r}$.

Soient $M$ un $\varphi$-réseau itéré dans $V,\left(\widehat{W}_{w}\right)_{w \in \underline{w}}$ une filtration de $\widehat{V}$ raffinant celle associée à $M$ et $w_{0}$ un élément de $\underline{w} \cap \underline{w}^{-}$.

On suppose que le bon sous-objet $\mathcal{E}_{w_{0}}^{M}=\mathcal{E}_{\widehat{W}_{w_{0}}}^{M}$ de $\mathcal{E}^{M}$ induit par $\widehat{W}_{w_{0}}$ vérifie l'inégalité

$$
\operatorname{deg} \mathcal{E}_{w_{0}}^{M}-\frac{w_{0}}{r} \operatorname{deg} \mathcal{E}^{M}>p\left(w_{0}\right) .
$$

Alors $M$ admet un transformé strict par $\widehat{W}_{w_{0}}$.

Démonstration. Supposons que ce ne soit pas le cas.

Alors la suite $M, M^{1}, M^{2}, \ldots, M^{m}, \ldots$ des transformés successifs de $M$ par $\widehat{W}_{w_{0}}$ doit vérifier

$$
\mathcal{E}_{w_{0}}^{M}=\mathcal{E}_{w_{0}}^{M^{1}}=\mathcal{E}_{w_{0}}^{M^{2}}=\cdots=\mathcal{E}_{w_{0}}^{M^{m}}=\cdots .
$$

Pour tout $m \geq 1$, l'inclusion $M^{m} \subset M$ induit un plongement $\mathcal{E}\left(M^{m}\right) \hookrightarrow \mathcal{E}(M)$ entre fibrés localement libres sur $X \otimes A$, et il résulte de l'égalité $\mathcal{E}_{w_{0}}^{M^{m}}=\mathcal{E}_{w_{0}}^{M}$ que le quotient $\mathcal{B}^{m}=\mathcal{E}(M) / \mathcal{E}\left(M^{m}\right)$ est un fibré localement libre de rang $r-w_{0}$ au-dessus $\operatorname{de} X \otimes A / \pi_{A}^{m} A$. 
On en déduit que, pour $m^{\prime} \geq m \geq 1, \mathcal{B}^{m}=\mathcal{B}^{m^{\prime}} / \pi_{A}^{m} \mathcal{B}^{m^{\prime}}$ si bien que le système projectif des $\mathcal{B}^{m}$ définit un quotient $\mathcal{B}$ de $\mathcal{E}(M)$ sur le complété formel de $X \otimes A$ le long de $X \otimes \kappa_{A}$ et donc aussi sur $X \otimes A$.

Le noyau $\mathcal{A}=\operatorname{Ker}[\mathcal{E}(M) \rightarrow \mathcal{B}]$ est un fibré localement libre sur $X \otimes A$ dont la fibre spéciale s'identifie à $\mathcal{E}_{w_{0}}^{M}$. Sa fibre sur $X \otimes K_{A}$ est un bon sous-objet de $\mathcal{E}$ qui a même rang $w_{0}$ et même degré que $\mathcal{E}_{w_{0}}^{M}$. Il y a contradiction.

Proposition 15. Soient $\left(\widehat{W}_{w}\right)_{w \in \underline{w}}$ une filtration de $\widehat{V}$ par des sous- $\varphi$-espaces, $w_{0}$ un élément de $\underline{w} \cap \underline{w}^{-}$et $\mu \geq 0$ une constante.

Alors, quitte à remplacer l'anneau de valuation discrète A par une extension finie non ramifiée ne dépendant que de ces données, la propriété suivante est vérifiée:

Pour tout $\varphi$-réseau itéré $M$ dans $V$ tel que

- la filtration de $\widehat{V}$ associée à $M$ est contenue dans $\left(\widehat{W}_{w}\right)_{w \in \underline{w}}$ et comprend l'élément $\widehat{W}_{w_{0}}$,

- $M$ n'admet pas de transformé réciproque par $\widehat{W}_{w_{0}}$, on a l'inégalité suivante entre pentes minimales et maximales

$$
\mu^{-}\left(\overline{\mathcal{E}}_{w_{0}}^{M}\right)+\mu \leq \max _{\substack{w \in w \\ w \leq w_{0}}} \mu^{+}\left(\mathcal{E}_{w}^{M} / \mathcal{E}_{w^{-}}^{M}\right)
$$

Démonstration. Pour tout $w<w_{0}$ dans $\underline{w}^{-}$, l'homomorphisme

$$
\Lambda^{1+w \tau} \mathcal{E}(M) \longrightarrow \Lambda^{1+w} \mathcal{E}^{\prime \prime}(M)
$$

induit par quotient un homomorphisme bien défini

$$
\Lambda^{1+w} \tau \mathcal{E}^{M} \longrightarrow \Lambda^{1+w} \mathcal{E}^{\prime \prime}(M) \otimes_{A} A / \pi_{A}^{q} A
$$

qui se factorise à travers l'homomorphisme surjectif

$$
\Lambda^{1+w} \tau \mathcal{E}^{M} \longrightarrow \operatorname{det}\left({ }^{\tau} \mathcal{E}^{M} / \overline{\mathcal{E}}_{w}^{M}\right) \otimes \overline{\mathcal{E}}_{w}^{M}
$$

si bien que par restriction on obtient une flèche

$$
\operatorname{det}\left({ }^{\tau} \mathcal{E}^{M} / \overline{\mathcal{E}}_{w}^{M}\right) \otimes \overline{\mathcal{E}}_{w_{0}}^{M} \longrightarrow \Lambda^{1+w} \mathcal{E}^{\prime \prime}(M) \otimes_{A} A / \pi_{A}^{q} A .
$$

Comme par hypothèse $M$ n'admet pas de transformé réciproque par $\widehat{W}_{w_{0}}$, il faut que l'un au moins de ces homomorphismes soit non nul. Autrement dit, il existe $w<w_{0}$ dans $\underline{w}^{-}$et $e \in \mathbb{N}, 1 \leq e \leq q$, pour lesquels on dispose maintenant d'un homomorphisme bien défini et non nul

$$
\operatorname{det}\left({ }^{\tau} \mathcal{E}^{M} / \overline{\mathcal{E}}_{w}^{M}\right) \otimes \overline{\mathcal{E}}_{w_{0}}^{M} \longrightarrow \Lambda^{1+w} \mathcal{E}^{\prime \prime}(M) \otimes_{A} \pi_{A}^{e-1} A / \pi_{A}^{e} A \cong \Lambda^{1+w} \mathcal{E}^{\prime M}
$$

dont on remarque que l'image $\mathcal{A}^{\prime \prime}$ est nécessairement contenue dans le sous-fibré $\operatorname{det}\left(\mathcal{E}_{w}^{\prime \prime M}\right) \otimes \mathcal{E}^{\prime \prime M} / \mathcal{E}_{w}^{\prime \prime M} \operatorname{de~} \Lambda^{1+w} \mathcal{E}^{M}$.

Soit $\mathcal{A}$ le fibré intersection de $\mathcal{A}^{\prime \prime}$ avec $\operatorname{det}\left(\mathcal{E}_{w}^{M}\right) \otimes \mathcal{E}^{M} / \mathcal{E}_{w}^{M} \operatorname{dans} \operatorname{det}\left(\mathcal{E}_{w}^{\prime M}\right) \otimes$ $\mathcal{E}^{\prime M} / \mathcal{E}_{w}^{\prime M}$. Ainsi on a

$$
\operatorname{deg}\left({ }^{\tau} \mathcal{E}^{M} / \overline{\mathcal{E}}_{w}^{M}\right)+\mu^{-}\left(\overline{\mathcal{E}}_{w_{0}}^{M}\right) \leq \mu^{-}\left(\mathcal{A}^{\prime \prime}\right) \leq \mu^{-}(\mathcal{A})+1 .
$$

D'autre part, il résulte encore de nos hypothèses que le fibré $\overline{\mathcal{A}}$ intersection de ${ }^{\tau} \mathcal{A}$ avec $\operatorname{det}\left({ }^{\tau} \mathcal{E}_{w}^{M}\right) \otimes \overline{\mathcal{E}}_{w_{0}}^{M}$ a un rang strictement inférieur à celui de ${ }^{\tau} \mathcal{A}$ avec donc $\mu^{-}(\mathcal{A})=\mu^{-}\left({ }^{\tau} \mathcal{A}\right) \leq \mu^{-}\left({ }^{\tau} \mathcal{A} / \overline{\mathcal{A}}\right)$. 
Or d'après le lemme 16 ci-dessous et quitte à avoir remplacé $A$ par une extension finie non ramifiée, il existe dans $X$ un sous-schéma fermé fini $I$ dont on a pu choisir le degré arbitrairement grand et tel que l'homomorphisme

$$
\operatorname{det}\left({ }^{\tau} \mathcal{E}^{M} / \overline{\mathcal{E}}_{w}^{M}\right) \otimes \overline{\mathcal{E}}_{w_{0}}^{M} \rightarrow \Lambda^{1+w} \mathcal{E}^{\prime \prime}(M) \otimes_{A} A / \pi^{q} A
$$

s'annule modulo $I$, si bien que le plongement

$$
{ }^{\tau} \mathcal{A} / \overline{\mathcal{A}} \hookrightarrow \operatorname{det}\left({ }^{\tau} \mathcal{E}_{w}^{M}\right) \otimes{ }^{\tau} \mathcal{E}^{M} /\left({ }^{\tau} \mathcal{E}_{w}^{M} \oplus \overline{\mathcal{E}}_{w_{0}}^{M}\right)
$$

est lui-même nul modulo $I$.

On en déduit

$$
\begin{aligned}
\mu^{-}\left({ }^{\tau} \mathcal{A} / \overline{\mathcal{A}}\right)+\operatorname{deg} I & \leq \operatorname{deg}\left({ }^{\tau} \mathcal{E}_{w}^{M}\right)+1+\mu^{-}\left({ }^{\tau} \mathcal{E}_{w_{0}}^{M} /{ }^{\tau} \mathcal{E}_{w}^{M}\right) \\
& \leq \operatorname{deg}\left({ }^{\tau} \mathcal{E}^{M} / \overline{\mathcal{E}}_{w}^{M}\right)+1+\mu^{-}\left(\mathcal{E}_{w_{0}}^{M} / \mathcal{E}_{w}^{M}\right),
\end{aligned}
$$

ce qui termine la démonstration si $\operatorname{deg} I \geq \mu+2$.

Lemme 16. Soit $\left(\widehat{W}_{w}\right)_{w \in \underline{w}}$ une filtration de $\widehat{V}$ par des sous- $\varphi$-espaces.

Soit $\{M\}$ la famille de tous les $\varphi$-réseaux itérés $M$ de $V$ relativement à une famille d'entiers $d_{1}^{M}, \ldots, d_{r-1}^{M} \geq 0$ tels que la filtration de $\widehat{V}$ associée soit contenue dans $\left(\widehat{W}_{w}\right)_{w \in \underline{w}}$.

Soit enfin $\bar{I} \hookrightarrow X$ un sous-schéma fermé fini évitant les prolongements à $\operatorname{Spec} A$ des zéro et pôle du chtouca $\left(\begin{array}{cccc}\mathcal{E} & \hookrightarrow & \mathcal{E}^{\prime} \\ & \nearrow & \\ \tau \mathcal{E} & & \end{array}\right)$.

Considérons les propriétés suivantes portant sur les $\varphi$-réseaux itérés $M$ dans $\{M\}$ :

(1) Aucun des homomorphismes induits

$$
u_{s}: \Lambda^{s \tau}\left(\mathcal{E}(M) \otimes \mathcal{O}_{I}\right) \longrightarrow \Lambda^{s}\left(\mathcal{E}(M) \otimes \mathcal{O}_{I}\right), \quad 1 \leq s \leq r,
$$

n'est nilpotent, même après réduction modulo $\pi_{A}$.

(2) Il existe une famille d'homomorphismes

$$
v_{s}: \Lambda^{s}\left(\mathcal{E}(M) \otimes \mathcal{O}_{I}\right) \longrightarrow \Lambda^{s}\left(A^{r} \otimes \mathcal{O}_{I}\right), \quad 1 \leq s \leq r,
$$

telle que, pour tout $s,{ }^{\tau} v_{s}=v_{s} \circ u_{s}$ et que, pour n'importe quel choix de base de $\mathcal{E}(M) \otimes \mathcal{O}_{I}$ sur l'anneau $A \otimes \mathcal{O}_{I}$, la famille $\left(v_{1}, v_{2}, \ldots, v_{r} ; \pi_{A}^{d_{1}^{M}}, \ldots, \pi_{A}^{d_{r-1}^{M}}\right)$ soit élément de $\Omega^{r}\left(A \otimes \mathcal{O}_{I}\right)$.

Alors:

(i) La propriété (2) entraîne la propriété (1).

D'autre part, si un seul des $\varphi$-réseaux itérés dans $\{M\}$ vérifie la propriété (1) [resp. (2)], il en est de même de tous les autres.

(ii) Quitte à remplacer A par une extension finie non ramifiée, la propriété (1) entraîne la propriété (2).

Démonstration. (i) La première assertion est évidente.

Pour ce qui est de la seconde, on remarque que si $M, M^{\prime}$ sont deux $\varphi$-réseaux itérés dans $\{M\}$ tels que $M^{\prime}$ soit le transformé de $M$ par l'un des $\widehat{W}_{w}$ alors $M$ vérifie la propriété (1) [resp. (2)] si et seulement si $M^{\prime}$ la vérifie. Or si $M, M^{\prime}$ sont maintenant deux éléments quelconques de $\{M\}$, on peut construire deux suites $M$, $M^{1}, M^{2}, \ldots, M^{m}$ et $M^{\prime}, M^{\prime 1}, M^{\prime 2}, \ldots, M^{\prime m^{\prime}}$ de transformés successifs de $M$ et $M^{\prime}$ par des $\widehat{W}_{w}$ telles que $M^{m}$ et $M^{\prime m^{\prime}}$ soient des $\varphi$-réseaux itérés relativement à une 
même suite d'entiers $d_{1}, d_{2}, \ldots, d_{r-1} \geq 0$ et donc coïncident d'après la proposition 2 (ii) du paragraphe 2a. La conclusion s'en déduit.

(ii) D'après (i) il suffit de vérifier l'assertion pour un élément fixé $M$ de $\{M\}$ vérifiant l'assertion (1).

Pour tout $s \geq 2$, on doit avoir

$$
\begin{aligned}
& u_{s}=\left(\prod_{1 \leq t<s} \pi_{A}^{-(s-t) d_{t}^{M}(q-1)}\right) \Lambda^{s} u_{1}, \\
& v_{s}=\left(\prod_{1 \leq t<s} \pi_{A}^{-(s-t) d_{t}^{M}}\right) \Lambda^{s} v_{1} .
\end{aligned}
$$

Il s'agit donc de déterminer $v_{1}$ en fonction de $u_{1}$.

Or $\mathcal{M}=\mathcal{E}(M) \otimes \mathcal{O}_{I}$ est un module libre de $\operatorname{rang} r$ sur l'anneau $A \otimes \mathcal{O}_{I}$. Il est muni d'une filtration $0=\mathcal{M}_{0} \varsubsetneqq \cdots \varsubsetneqq \mathcal{M}_{w} \varsubsetneqq \cdots \varsubsetneqq \mathcal{M}_{r}=\mathcal{M}$ respectée par $u_{1}$ et telle que

- chaque $\mathcal{M}_{w} / \mathcal{M}_{w^{-}}, w \in \underline{w}$, est libre de rang $w-w^{-}$sur l'anneau $A \otimes \mathcal{O}_{I}$,

- pour tout $w \in \underline{w}$, l'homomorphisme $\left(\prod_{t \leq w^{-}} \pi_{A}^{-d_{t}^{M}(q-1)}\right) u_{1}$ induit un isomorphisme de ${ }^{\tau}\left(\mathcal{M}_{w} / \mathcal{M}_{w^{-}}\right)$sur $\mathcal{M}_{w} / \mathcal{M}_{w^{-}}$.

Quitte à remplacer $A$ par une extension finie non ramifiée, on peut donc choisir pour chaque $\mathcal{M}_{w} / \mathcal{M}_{w^{-}}$une base $\left\{\bar{m}_{s}, w^{-}+1 \leq s \leq w\right\}$ dont tous les vecteurs vérifient la relation

$$
u_{1}\left({ }^{\tau} \bar{m}_{s}\right)=\left(\prod_{t \leq w^{-}} \pi_{A}^{d_{t}^{M}(q-1)}\right) \bar{m}_{s}
$$

puis relever chaque $\bar{m}_{s}$ en un élément $m_{s} \in \mathcal{M}_{w}$ vérifiant

$$
u_{1}\left({ }^{\tau} m_{s}\right)=\left(\prod_{t \leq w^{-}} \pi_{A}^{d_{t}^{M}(q-1)}\right) m_{s} .
$$

Alors l'isomorphisme $v_{1}$ de $\mathcal{M}=\mathcal{E}(M) \otimes \mathcal{O}_{I}$ sur $A^{r} \otimes \mathcal{O}_{I}$ qui envoie la base $\left\{m_{1}, m_{2}, \ldots, m_{r}\right\}$ sur la base canonique de $A^{r} \otimes \mathcal{O}_{I}$ répond à la question posée.

De la proposition $15 \mathrm{du}$ présent paragraphe combinée avec la proposition $11 \mathrm{du}$ paragraphe précédent, on déduit:

Corollaire 17. Soient $M$ un $\varphi$-réseau itéré dans $V,\left(\widehat{W}_{w}\right)_{w \in \underline{w}}$ une filtration de $\widehat{V}$ raffinant celle associée à $M$ et $w_{0}$ un élément de $\underline{w} \cap \underline{w}^{-}$.

Alors, quitte à remplacer l'anneau de valuation discrète A par une extension finie non ramifiée:

(i) Ou bien $M$ admet un modifié par $\widehat{W}_{w_{0}}$ qui ne compte pas $\widehat{W}_{w_{0}}$ dans sa filtration de $\widehat{V}$ associée, ou bien $M$ admet un transformé réciproque strict par $\widehat{W}_{w_{0}}$.

(ii) A fortiori $M$ admet un modifié maximal par $\widehat{W}_{w_{0}}$ et il admet un modifié maximal par les $\widehat{W}_{w}, w \geq w_{0}$.

Puis on obtient:

Corollaire 18. Soient $M$ un $\varphi$-réseau itéré dans $V,\left(\widehat{W}_{w}\right)_{w \in \underline{w}}$ une filtration $d e \widehat{V}$ raffinant celle associée à $M$ et $w_{0}$ un élément de $\underline{w} \cap \underline{w}^{-}$. 
On suppose que les hypothèses de la proposition 14 sont vérifiées et que pour tout $w>w_{0}$ dans $\underline{w}^{-}$tel que $\widehat{W}_{w}$ figure dans la filtration associée à $M$, on ait $\overline{\mathcal{E}}_{w}^{M} \oplus{ }^{\tau} \mathcal{E}_{w}^{M} \varsubsetneqq \tau \mathcal{E}^{M}$ et $\overline{\mathcal{E}}_{w}^{M}+{ }^{\tau} \mathcal{E}_{w^{+}}^{M}={ }^{\tau} \mathcal{E}^{M}$.

Alors, quitte à remplacer l'anneau de valuation discrète A par une extension finie non ramifiée, $M$ admet un transformé strict par les $\widehat{W}_{w}, w \geq w_{0}$.

Démonstration. D'après le corollaire 17 et la proposition $14 \mathrm{du}$ présent paragraphe combinés avec la proposition 12 du précédent paragraphe et quitte à remplacer $A$ par une extension finie non ramifiée, $M=M^{0}$ admet un transformé $M^{1}$ par les $\widehat{W}_{w}, w \geq w_{0}$; si $M^{1}$ est un transformé strict, on a terminé et sinon il vérifie les mêmes propriétés que $M$ si bien qu'il admet un transformé $M^{2}$ par les $\widehat{W}_{w}, w \geq w_{0}$.

En répétant cette opération autant de fois que nécessaire, on finit nécessairement par obtenir un transformé strict de $M$ par les $\widehat{W}_{w}, w \geq w_{0}$. En effet, si ce n'était le cas, on aurait construit une suite infinie de $\varphi$-réseaux itérés $\left(M^{m}\right)_{m>0}$ relativement à des suites d'entiers $d_{1}^{M^{m}}, \ldots, d_{r-1}^{M^{m}} \geq 0$ telles que pour tout $m$ on ait $d_{s}^{M^{m+1}} \leq$ $d_{s}^{M^{m}}, \forall s \geq w$, et $\sum_{s \geq w} d_{s}^{M^{m+1}}<\sum_{s \geq w} d_{s}^{M^{m}} ;$ c'est impossible.

De même, par combinaison avec la proposition 13 du précédent paragraphe, on obtient:

Corollaire 19. Soient $M$ un $\varphi$-réseau itéré dans $V,\left(\widehat{W}_{w}\right)_{w \in \underline{w}}$ une filtration de $\widehat{V}$ raffinant celle associée à $M$ et $w_{0}$ un élément de $\underline{w} \cap \underline{w}^{-}$.

On suppose que pour toute extension finie non ramifiée $A^{\prime}$ de $A$ et pour tout $\varphi$-réseau itéré $M^{\prime}$ de $V \otimes_{K_{A_{X}}} K_{A_{X}^{\prime}}$ dont la filtration de $\widehat{V} \otimes_{K_{\widehat{A}_{X}}} K_{\widehat{A}_{X}^{\prime}}$ associée soit contenue dans $\left(\widehat{W}_{w} \otimes_{K_{\widehat{A}_{X}}} K_{\widehat{A}_{X}^{\prime}}\right)_{w \in \underline{w}}$, cette filtration comprenne l'élément $\widehat{W}_{w_{0}} \otimes_{K_{\widehat{A}_{X}}}$ $K_{\widehat{A}_{X}^{\prime}}$. On suppose aussi que pour tout $w>w_{0}$ dans $\underline{w}^{-}$tel que $\widehat{W}_{w}$ figure dans la filtration associée à $M$, on ait $\overline{\mathcal{E}}_{w}^{M} \oplus{ }^{\tau} \mathcal{E}_{w}^{M} \varsubsetneqq \tau \mathcal{E}^{M}$ et $\overline{\mathcal{E}}_{w}^{M}+{ }^{\tau} \mathcal{E}_{w^{+}}^{M}={ }^{\tau} \mathcal{E}^{M}$.

Alors, quitte à remplacer $A$ par une extension finie non ramifiée, $M$ admet un transformé réciproque strict par les $\widehat{W}_{w}, w \geq w_{0}$.

e) Dégénérescence des chtoucas en chtoucas itérés. Nous allons démontrer dans ce paragraphe:

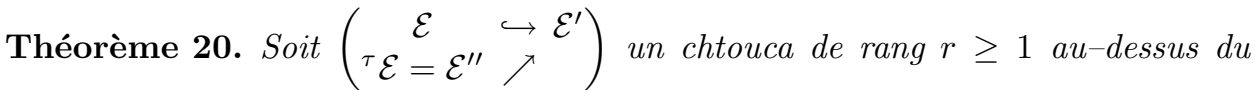
point générique Spec $K_{A}$ d'un anneau de valuation discrète $A$ contenant $\mathbb{F}_{q}$. Soit $V$ sa fibre générique.

Soit $p:[O, r] \rightarrow \mathbb{R}_{+}$un polygone qui est 2 -grand et tel que le morphisme Spec $K_{A}$ $\longrightarrow \mathrm{Cht}^{r}$ correspondant au chtouca considéré se factorise à travers l'ouvert $\mathrm{Cht}^{r, \bar{p}} \leq p$. Alors:

(i) Quitte à remplacer $A$ par une extension finie, il existe dans $V$ un $\varphi$-réseau itéré $M$ dont le chtouca dégénéré associé soit un chtouca itéré et même définisse un objet de $\overline{\mathrm{Cht}^{r, \bar{p} \leq p}}(\operatorname{Spec} A)$.

(ii) Si $M$ et $M^{\prime}$ sont deux $\varphi$-réseaux itérés de $V$ définissant chacun un objet de $\overline{\mathrm{Cht}^{r, \bar{p}} \leq p}(\operatorname{Spec} A)$, on a nécessairement $M=M^{\prime}$.

Démonstration du théorème 20 (i). Tout d'abord et quitte à remplacer $A$ par une extension finie, on sait d'après la proposition $4 \mathrm{du}$ paragraphe $2 \mathrm{a}$ qu'existe dans $V$ un $\varphi$-réseau itéré $M$. 
On peut supposer que le type $\underline{r}=\left(r_{1}, \ldots, r_{k}\right)$ de $M$ est maximal pour l'ordre lexicographique. Autrement dit, pour toute extension finie $A^{\prime}$ de $A$ et pour tout $\varphi$-réseau itéré $M^{\prime}$ de $V \otimes_{K_{A_{X}}} K_{A_{X}^{\prime}}$, le type $\underline{r}^{\prime}=\left(r_{1}^{\prime}, \ldots, r_{k^{\prime}}^{\prime}\right)$ de $M^{\prime}$ vérifie

$$
\begin{aligned}
& r_{1}^{\prime} \leq r_{1}, \\
& r_{2}^{\prime} \leq r_{2} \text { si } r_{1}^{\prime}=r_{1}, \\
& r_{3}^{\prime} \leq r_{3} \text { si } r_{2}^{\prime}=r_{2}, r_{1}^{\prime}=r_{1},
\end{aligned}
$$

etc.

Notons $\left(\widehat{V}_{s}\right)_{s \in \underline{r}}$ la filtration de $\widehat{V}$ associée à $M$.

D'autre part, pour tout entier $r^{\prime}, 0 \leq r^{\prime} \leq r$, notons $d\left(r^{\prime}\right) \in \mathbb{Z}$ l'unique entier tel que

$$
p\left(r^{\prime}\right)-1<d\left(r^{\prime}\right)-\frac{r^{\prime}}{r} \operatorname{deg} \mathcal{E} \leq p\left(r^{\prime}\right) .
$$

Nous allons maintenant procéder à une construction par étapes, sans plus préciser qu'à chaque pas on remplace $A$ par une extension finie.

Il existe dans $V$ un $\varphi$-réseau itéré $M^{0}$ tel que

- la filtration de $\widehat{V}$ associée à $M^{0}$ n'est autre que $\left(\widehat{V}_{s}\right)_{s \in r}$,

- le chtouca dégénéré induit par $M^{0}$ est un chtouca itéré,

- pour tout $s \in \underline{r} \cap \underline{r}^{-}, \operatorname{deg} \mathcal{E}_{s}^{M^{0}}=d(s)+1$.

Cela résulte du lemme suivant:

Lemme 21. Soient $N$ un $\varphi$-réseau itéré de $V,\left(\widehat{W}_{w}\right)_{w \in \underline{w}}$ la filtration de $\widehat{V}$ associée et $w_{0}$ un élément de $\underline{w}$ tels que

- les deux sous-familles $\left\{\widehat{W}_{w}, w<w_{0}\right\}$ et $\left\{\widehat{V}_{s}, s<w_{0}\right\}$ se confondent,

- pour tout $w \geq w_{0}$ dans $\underline{w} \cap \underline{w}^{-}$, on a

$$
\operatorname{deg} \mathcal{E}_{w}^{N}=d(w), \quad{ }^{\tau} \mathcal{E}_{w}^{N} \oplus \overline{\mathcal{E}}_{w}^{N} \varsubsetneqq{ }^{\tau} \mathcal{E}^{N} \quad \text { et } \quad{ }^{\tau} \mathcal{E}_{w^{+}}^{N}+\overline{\mathcal{E}}_{w}^{N}={ }^{\tau} \mathcal{E}^{N} .
$$

Alors il existe un $\varphi$-réseau itéré $N^{\prime}$ de $V$ tel que

- la filtration de $\widehat{V}$ associée à $N^{\prime}$ contient $\left(\widehat{V}_{s}\right)_{s<w_{0}}$ et elle est contenue dans $\left(\widehat{W}_{w}\right)_{w \in \underline{w}}$

- le chtouca dégénéré induit par $N^{\prime}$ est un chtouca itéré,

- pour tout élément $w$ de $\underline{w}$, on a

$$
\begin{array}{ll}
\operatorname{deg} \mathcal{E}_{w}^{N^{\prime}}=d(w)+1 & \text { si } w<w_{0}, \\
\operatorname{deg} \mathcal{E}_{w}^{N^{\prime}}=d(w) & \text { si } w \geq w_{0} .
\end{array}
$$

Démonstration du lemme 21. On construit une suite de $\varphi$-réseaux itérés $N=N_{w_{0}}$, $N_{w_{0}^{-}}^{\prime}, N_{w_{0}^{-}}, \ldots$ dans $V$ de la manière suivante:

Si $w<w_{0}$ et $N_{w^{+}}$est déjà construit, on remplace celui-ci par son transformé réciproque strict par les $\widehat{W}_{w^{\prime}}, w^{\prime} \geq w$, puis on recommence autant de fois que nécessaire jusqu'à obtenir un $\varphi$-réseau itéré $N_{w}^{\prime}$ vérifiant $\operatorname{deg} \mathcal{E}_{w}^{N_{w}^{\prime}}>d(w)+1$. Une telle construction est possible d'après le corollaire $19 \mathrm{du}$ paragraphe $2 \mathrm{~d}$.

Puis on remplace $N_{w}^{\prime}$ par son transformé strict par les $\widehat{W}_{w^{\prime}}, w^{\prime} \geq w$, et on recommence autant de fois que nécessaire jusqu'à obtenir un $\varphi$-réseau itéré $N_{w}$ vérifiant $\operatorname{deg} \mathcal{E}_{w}^{N_{w}}=d(w)+1$. C'est possible d'après le corollaire $18 \mathrm{du}$ paragraphe $2 \mathrm{~d}$.

Il résulte des propositions 13 et 12 du paragraphe 2 c que $N^{\prime}=N_{0^{+}}$répond à la question posée. 
Suite de la démonstration du théorème 20 (i). Partant du $\varphi$-réseau itéré $M^{0}$, nous allons construire maintenant une suite finie de $\varphi$-réseaux itérés $M^{n}$ et d'éléments $w^{n} \in\{1,2, \ldots, r\}$ tels que:

- Si $\left(\widehat{W}_{w}^{n}\right)_{w \in \underline{w}^{n}}$ désigne la filtration de $\widehat{V}$ associée à $M^{n}$, on a $w^{n} \in \underline{w}^{n}$ et $\left\{\widehat{W}_{w}^{n}, w<w^{n}\right\}=\left\{\widehat{V}_{s}, s<w^{n}\right\}$.

- Le chtouca dégénéré induit par $M^{n}$ est un chtouca itéré.

- Pour tout élément $w$ de $\underline{w}^{n}$, on a

$$
\begin{array}{ll}
\operatorname{deg} \mathcal{E}_{w}^{M^{n}}=d(w)+1 & \text { si } w<w^{n}, \\
\operatorname{deg} \mathcal{E}_{w}^{M^{n}}=d(w) & \text { si } w \geq w^{n} .
\end{array}
$$

- Pour tout bon sous-objet $\mathcal{A}$ de $\mathcal{E}^{M^{n}}$ vérifiant $\mathcal{E}_{w}^{M^{n}} \varsubsetneqq \mathcal{A} \varsubsetneqq \mathcal{E}_{w^{+}}^{M^{n}}$ avec $w \in \underline{w}^{n}$, $w \geq w^{n}$, on a

$$
\operatorname{deg} \mathcal{A} \leq d(\operatorname{rg} \mathcal{A})
$$

et même

$$
\operatorname{deg} \mathcal{A}<d(\operatorname{rg} \mathcal{A}) \quad \text { si }{ }^{\tau} \mathcal{A}+\overline{\mathcal{E}}_{w}^{M^{n}} \varsubsetneqq{ }^{\tau} \mathcal{E}^{M^{n}} .
$$

Le passage du couple $\left(M^{n}, w^{n}\right)$ au couple $\left(M^{n+1}, w^{n+1}\right)$ se fait de la manière suivante:

On choisit un bon sous-objet $\mathcal{A}$ de $\mathcal{E}^{M^{n}}$ vérifiant

$$
\mathcal{E}_{w^{n}-}^{M^{n}} \subseteq \mathcal{A} \varsubsetneqq \mathcal{E}_{w^{n}}^{M^{n}} \quad \text { et } \quad \operatorname{deg} \mathcal{A}>d(\operatorname{rg} \mathcal{A})
$$

et qui soit de rang maximal $w^{n+1}$ parmi ceux satisfaisant ces propriétés. (Il n'est pas nécessaire de se préoccuper des sous-objets $\mathcal{A}$ vérifiant $\mathcal{E}_{w^{n_{-}}}^{M^{n}} \subseteq \mathcal{A} \varsubsetneqq \mathcal{E}_{w^{n}}^{M^{n}}$, ${ }^{\tau} \mathcal{A}+\overline{\mathcal{E}}_{w^{n}-}^{M^{n}} \varsubsetneqq{ }^{\tau} \mathcal{E}^{M^{n}}$ et $\operatorname{deg} \mathcal{A}=d(\operatorname{rg} \mathcal{A})$ car dans nos transformations leur degré va diminuer automatiquement.) Ce bon sous-objet $\mathcal{A}$ correspond à un sous- $\varphi-$ espace $\widehat{W}_{w^{n+1}}^{n}$ de $\widehat{V}$ compatible avec la filtration $\left(\widehat{W}_{w}^{n}\right)_{w \in \underline{w}^{n}}$. On construit alors le transformé strict de $M^{n}$ par les $\widehat{W}_{w}^{n}, w \geq w^{n+1}$, et on recommence autant de fois que nécessaire jusqu'à obtenir un $\varphi$-réseau itéré $M^{\prime n+1}$ vérifiant $\operatorname{deg} \mathcal{E}_{w^{n+1}}^{M^{\prime n+1}}=d\left(w^{n+1}\right)$. Puis on applique à $M^{\prime n+1}$ et $w^{n+1}$ la construction du lemme 21, d'où un nouveau $\varphi$-réseau itéré $M^{n+1}$.

Il résulte de la proposition 12 du paragraphe $2 \mathrm{c}$ et de la proposition 9 (ii) du paragraphe $2 \mathrm{~b}$ que la suite des $\left(M^{n}, w^{n}\right)$ vérifie bien les propriétés annoncées.

La suite des $w^{n}$ est strictement décroissante, donc la construction s'arrête au bout d'un nombre fini $m$ de pas et on voit que le $\varphi$-réseau itéré $M^{m}$ répond à la question posée.

Afin de démontrer la partie (ii) du théorème 20, on a besoin du lemme évident:

Lemme 22. Soient $M$ et $M^{\prime}$ deux $\varphi$-réseaux itérés de $V$ relativement à des familles d'entiers $d_{1}, d_{2}, \ldots, d_{r-1} \geq 0$ et $d_{1}^{\prime}, d_{2}^{\prime}, \ldots, d_{r-1}^{\prime} \geq 0$, de types $\underline{r}=\left(r_{1}, \ldots, r_{k}\right)$ et $\underline{r}^{\prime}=\left(r_{1}^{\prime}, \ldots, r_{k^{\prime}}^{\prime}\right)$.

Alors le réseau $M^{\prime \prime}=M+M^{\prime}$ est lui-même un $\varphi$-réseau itéré de $V$, relativement à une famille d'entiers $d_{1}^{\prime \prime}, d_{2}^{\prime \prime}, \ldots, d_{r-1}^{\prime \prime} \geq 0$ et d'un type $\underline{r}^{\prime \prime}=\left(r_{1}^{\prime \prime}, \ldots, r_{k^{\prime \prime}}^{\prime \prime}\right)$.

De plus, si $\left(\widehat{V}_{s}\right)_{s \in \underline{r}}$, $\left(\widehat{V}_{s}^{\prime}\right)_{s \in \underline{r}^{\prime}}$ et $\left(\widehat{V}_{s}^{\prime \prime}\right)_{s \in \underline{r}^{\prime \prime}}$ désignent les filtrations de $\widehat{V}$ canoniquement associées à $M, M^{\prime}$ et $M^{\prime \prime}$ et si pour tout entier $d$, on note $s_{d}$, $s_{d}^{\prime}$ et $s_{d}^{\prime \prime}$ les 
plus grands éléments de $\underline{r}, \underline{r}^{\prime}$ et $\underline{r}^{\prime \prime}$ respectivement tels que

$$
\sum_{\substack{s \in \underline{r}_{d} \\ s<s_{d}}} d_{s} \leq d, \quad \sum_{\substack{s \in \underline{r}^{\prime} \\ s<\bar{s}_{d}^{\prime}}} d_{s}^{\prime} \leq d, \quad \sum_{\substack{s \in \underline{r}^{\prime \prime} \\ s<s_{d}^{\prime \prime}}} d_{s}^{\prime \prime} \leq d
$$

on $a \widehat{V}_{s_{d}^{\prime \prime}}^{\prime \prime}=\widehat{V}_{s_{d}}+\widehat{V}_{s_{d}^{\prime}}^{\prime}$

Démonstration du théorème 20 (ii). Soient donc $M$ et $M^{\prime}$ deux $\varphi$-réseaux itérés de $V$ définissant chacun un objet de $\overline{\mathrm{Cht}^{r, \bar{p}} \leq p}(\operatorname{Spec} A)$.

On se sert des notations du lemme 22 et particulièrement du $\varphi$-réseau itéré $M^{\prime \prime}=M+M^{\prime}$.

Afin de prouver que $M=M^{\prime}$, il suffit de démontrer que les deux homomorphismes $\mathcal{E}^{M} \rightarrow \mathcal{E}^{M^{\prime \prime}}, \mathcal{E}^{M^{\prime}} \rightarrow \mathcal{E}^{M^{\prime \prime}}$ induits par les inclusions $M \subseteq M^{\prime \prime}, M^{\prime} \subseteq M^{\prime \prime}$, sont des isomorphismes ou, ce qui revient au même, qu'ils sont injectifs.

Montrons par exemple que l'homomorphisme $\mathcal{E}^{M} \rightarrow \mathcal{E}^{M^{\prime \prime}}$ est injectif.

Pour tout entier $d$, on dispose de deux homomorphismes induits $\mathcal{E}_{s_{d}}^{M} / \mathcal{E}_{s_{d}^{-}}^{M} \rightarrow$ $\mathcal{E}_{s_{d}^{\prime \prime}}^{M^{\prime \prime}} / \mathcal{E}_{s_{d}^{\prime \prime-}}^{M^{\prime \prime}}$ et $\mathcal{E}_{s_{d}^{\prime}}^{M^{\prime}} / \mathcal{E}_{s_{d}^{\prime-}}^{M^{\prime}} \rightarrow \mathcal{E}_{s_{d}^{\prime \prime}}^{M^{\prime \prime}} / \mathcal{E}_{s_{d}^{\prime \prime}}^{M^{\prime \prime}}$ dont les images engendrent $\mathcal{E}_{s_{d}^{\prime \prime}}^{M^{\prime \prime}} / \mathcal{E}_{s_{d}^{\prime \prime-}}^{M^{\prime \prime}}$ en le point générique de $X \otimes \kappa_{A}$.

Notons $\mathcal{E}_{t_{d}}^{M} / \mathcal{E}_{s_{d}^{-}}^{M}$ le noyau de $\mathcal{E}_{s_{d}}^{M} / \mathcal{E}_{s_{d}^{-}}^{M} \rightarrow \mathcal{E}_{s_{d}^{\prime \prime}}^{M^{\prime \prime}} / \mathcal{E}_{s_{d}^{\prime \prime}}^{M{ }^{\prime \prime}}, \mathcal{E}_{t_{d}^{\prime \prime}}^{M^{\prime \prime}} / \mathcal{E}_{s_{d}^{\prime \prime}}^{M^{\prime \prime}}$ le sous-fibré maximal de $\mathcal{E}_{s_{d}^{\prime \prime}}^{M^{\prime \prime}} / \mathcal{E}_{s_{d}^{\prime \prime-}}^{M^{\prime \prime}}$ engendré par son image et $\mathcal{E}_{t_{d}^{\prime}}^{M^{\prime}} / \mathcal{E}_{s_{d}^{\prime-}}^{M^{\prime}}$ le noyau du composé $\mathcal{E}_{s_{d}^{\prime}}^{M^{\prime}} / \mathcal{E}_{s_{d}^{\prime-}}^{M^{\prime}} \rightarrow \mathcal{E}_{s_{d}^{\prime \prime}}^{M^{\prime \prime}} / \mathcal{E}_{s_{d}^{\prime \prime}}^{M^{\prime \prime}} \rightarrow \mathcal{E}_{s_{d}^{\prime \prime}}^{M^{\prime \prime}} / \mathcal{E}_{t_{d}^{\prime \prime}}^{M^{\prime \prime}}$

Ainsi on dispose de plongements

$$
\mathcal{E}_{s_{d}}^{M} / \mathcal{E}_{t_{d}}^{M} \hookrightarrow \mathcal{E}_{t_{d}^{\prime \prime}}^{M^{\prime \prime}} / \mathcal{E}_{s_{d}^{\prime \prime}}^{M^{\prime \prime}} \quad \text { et } \quad \mathcal{E}_{s_{d}^{\prime}}^{M^{\prime}} / \mathcal{E}_{t_{d}^{\prime}}^{M^{\prime}} \hookrightarrow \mathcal{E}_{s_{d}^{\prime \prime}}^{M^{\prime \prime}} / \mathcal{E}_{t_{d}^{\prime \prime}}^{M^{\prime \prime}}
$$

qui sont des isomorphismes en le point générique de $X \otimes \kappa_{A}$, avec donc $s_{d}-t_{d}=$ $t_{d}^{\prime \prime}-s_{d}^{\prime \prime-}$ et $s_{d}^{\prime}-t_{d}^{\prime}=s_{d}^{\prime \prime}-t_{d}^{\prime \prime}$.

Pour tout entier $r^{\prime}, 0 \leq r^{\prime} \leq r$, notons à nouveau $d\left(r^{\prime}\right)$ l'unique entier vérifiant l'encadrement

$$
p\left(r^{\prime}\right)-1<d\left(r^{\prime}\right)-\frac{r^{\prime}}{r} \operatorname{deg} \mathcal{E} \leq p\left(r^{\prime}\right) .
$$

Comme $M$ et $M^{\prime}$ définissent des objets de $\overline{\mathrm{Cht}^{r, \bar{p}} \leq p}(\operatorname{Spec} A)$, on a pour tout entier $d$

$$
\operatorname{deg} \mathcal{E}_{s_{d}}^{M}=d\left(s_{d}\right), \operatorname{deg} \mathcal{E}_{t_{d}}^{M} \leq d\left(t_{d}\right), \operatorname{deg} \mathcal{E}_{s_{d}^{\prime}}^{M^{\prime}}=d\left(s_{d}^{\prime}\right), \operatorname{deg} \mathcal{E}_{t_{d}^{\prime}}^{M^{\prime}} \leq d\left(t_{d}^{\prime}\right),
$$

d'où on tire

$$
\begin{aligned}
& \operatorname{deg} \mathcal{E}_{t_{d}^{\prime \prime}}^{M^{\prime \prime}} / \mathcal{E}_{s_{d}^{\prime \prime}}^{M^{\prime \prime}} \geq d\left(s_{d}\right)-d\left(t_{d}\right) \geq d\left(t_{d}^{\prime \prime}\right)-d\left(s_{d}^{\prime \prime-}\right), \\
& \operatorname{deg} \mathcal{E}_{s_{d}^{\prime \prime}}^{M^{\prime \prime}} / \mathcal{E}_{t_{d}^{\prime \prime}}^{M^{\prime \prime}} \geq d\left(s_{d}^{\prime}\right)-d\left(t_{d}^{\prime}\right) \geq d\left(s_{d}^{\prime \prime}\right)-d\left(t_{d}^{\prime \prime}\right)
\end{aligned}
$$

puisque le polygone $p$ est 2 -grand et que, d'après le lemme 22 on a nécessairement $t_{d} \leq s_{d}^{\prime \prime-}$ et $s_{d}^{\prime} \leq s_{d}^{\prime \prime}$. De plus, la première [resp. la seconde] inégalité est stricte si $s_{d}-t_{d}=t_{d}^{\prime \prime}-s_{d}^{\prime \prime-}>0$ et $t_{d}<s_{d}^{\prime \prime-}\left[\right.$ resp. si $s_{d}^{\prime}-t_{d}^{\prime}=s_{d}^{\prime \prime}-t_{d}^{\prime \prime}>0$ et $\left.s_{d}^{\prime}<s_{d}^{\prime \prime}\right]$. Or on a

$$
\sum_{s \in \underline{r}^{\prime \prime}} \operatorname{deg} \mathcal{E}_{s}^{M^{\prime \prime}} / \mathcal{E}_{s^{-}}^{M^{\prime \prime}}=\operatorname{deg} \mathcal{E}^{M^{\prime \prime}}=\operatorname{deg} \mathcal{E}=d(r)=\sum_{s \in \underline{r}^{\prime \prime}} d(s)-d\left(s^{-}\right)
$$


d'où on voit que pour tout entier $d$ :

$$
\begin{gathered}
\operatorname{deg} \mathcal{E}_{t_{d}}^{M}=d\left(t_{d}\right), \quad \operatorname{deg} \mathcal{E}_{t_{d}^{\prime}}^{M^{\prime}}=d\left(s_{d}^{\prime}\right), \\
\operatorname{deg} \mathcal{E}_{s_{d}^{\prime \prime-}}^{M^{\prime \prime}}=d\left(s_{d}^{\prime \prime-}\right), \quad \operatorname{deg} \mathcal{E}_{t_{d}^{\prime \prime}}^{M^{\prime \prime}}=d\left(t_{d}^{\prime \prime}\right), \quad \operatorname{deg} \mathcal{E}_{s_{d}^{\prime \prime}}^{M^{\prime \prime}}=d\left(s_{d}^{\prime \prime}\right),
\end{gathered}
$$

et

$$
\begin{array}{cc}
s_{d}-t_{d}=t_{d}^{\prime \prime}-s_{d}^{\prime \prime-}>0 & \Longrightarrow t_{d}=s_{d}^{\prime \prime-}, s_{d}=t_{d}^{\prime \prime}, \\
s_{d}^{\prime}-t_{d}^{\prime}=s_{d}^{\prime \prime}-t_{d}^{\prime \prime}>0 & \Longrightarrow s_{d}^{\prime}=s_{d}^{\prime \prime}, t_{d}^{\prime}=t_{d}^{\prime \prime} .
\end{array}
$$

Raisonnant par l'absurde, supposons que l'homomorphisme $\mathcal{E}^{M} \rightarrow \mathcal{E}^{M^{\prime \prime}}$ ne soit pas injectif. D'après le lemme $22, \mathcal{E}_{s_{0}}^{M} \rightarrow \mathcal{E}_{s_{0}^{\prime \prime}}^{M^{\prime \prime}}$ est automatiquement injectif et donc il existe alors un entier $d \geq 1$ tel que $\mathcal{E}_{s_{d-1}}^{M} \rightarrow \mathcal{E}_{s_{d-1}^{\prime \prime}}^{M^{\prime \prime}}$ soit injectif mais que $\mathcal{E}_{s_{d}}^{M} / \mathcal{E}_{s_{d}^{-}}^{M} \rightarrow \mathcal{E}_{s_{d}^{\prime \prime}}^{M^{\prime \prime}} / \mathcal{E}_{s_{d}^{\prime \prime}}^{M \prime \prime}$ ne le soit pas, avec ainsi $s_{d}^{-}<t_{d} \leq s_{d}$ et $s_{e}^{-}=t_{e}$ si $e<d$.

Ainsi on a $\mathcal{E}_{s_{d}^{-}}^{M} \subsetneq \mathcal{E}_{t_{d}}^{M} \subseteq \mathcal{E}_{s_{d}}^{M}$ avec $\operatorname{deg} \mathcal{E}_{t_{d}}^{M}=d\left(t_{d}\right)$ d'où nécessairement ${ }^{\tau} \mathcal{E}_{t_{d}}^{M}+$ $\overline{\mathcal{E}}_{s_{d}^{-}}^{M}={ }^{\tau} \mathcal{E}^{M}$ et

$$
\operatorname{deg}\left({ }^{\tau} \mathcal{E}_{t_{d}}^{M} /{ }^{\tau} \mathcal{E}_{t_{d}}^{M} \cap \overline{\mathcal{E}}_{s_{d}^{-}}^{M}\right)=\operatorname{deg}\left(\mathcal{E}_{s_{d}^{-}}^{M}\right)+1=d\left(s_{d}^{-}\right)+1 .
$$

Mais d'autre part, on a $s_{d}^{-}=s_{d-1}=t_{d-1}^{\prime \prime}$ et on dispose de la suite de plongements suivants entre fibrés de même rang

$$
{ }^{\tau} \mathcal{E}_{s_{d-1}}^{M}={ }^{\tau} \mathcal{E}_{s_{d}^{-}}^{M} \hookrightarrow{ }^{\tau} \mathcal{E}_{t_{d}}^{M} /{ }^{\tau} \mathcal{E}_{t_{d}}^{M} \cap \overline{\mathcal{E}}_{s_{d}^{-}}^{M} \hookrightarrow{ }^{\tau} \mathcal{E}_{t_{d-1}^{\prime \prime}}^{M^{\prime \prime}} .
$$

Comme $\operatorname{deg}\left({ }^{\tau} \mathcal{E}_{t_{d-1}^{\prime \prime}}^{M^{\prime \prime}}\right)=d\left(t_{d-1}^{\prime \prime}\right)=d\left(s_{d}^{-}\right)$, il y a contradiction.

Ceci termine la démonstration.

Remarque de conclusion. Le théorème $12 \mathrm{du}$ paragraphe $1 \mathrm{~d}$ résulte du critère valuatif tel qu'énoncé dans le théorème 20 du présent paragraphe: cela se voit en faisant une récurrence sur le rang $r$ et employant la proposition $7 \mathrm{du}$ paragraphe $1 \mathrm{c}$.

\section{BiBLIOGRAPHIE}

1. C. De Concini et C. Procesi, Complete symmetric varieties, in Invariant Theory, Montecatini 1982, Lecture notes in Mathematics 996, Springer-Verlag. MR 85e:14070

2. V.G. Drinfeld, The proof of Petersson's conjecture for GL(2) over a global field of characteristic $p$, Functional analysis and its applications 22, 1988. MR 90c:11038

3. V.G. Drinfeld, Cohomology of compactified manifolds of F-sheaves of rank 2, Journal of Soviet Mathematics 46, 1989. MR 89b:11050

4. L. Lafforgue, Chtoucas de Drinfeld et conjecture de Ramanujan-Petersson, Astérisque $\mathrm{n}^{\circ} 243$, Société Mathématique de France, 1997. CMP 98:07

5. D. Laksov, Completed quadrics and linear maps, in Algebraic geometry, Bowdoin 1985, Proceedings of symposia in pure mathematics, volume 46 II, 1987. MR 89c:14077

6. S.G. Langton, Valuative criteria for families of vector bundles on algebraic varieties, Annals of Mathematics 101, 1975. MR 51:510

URA D0752 du CNRS, Université de PARIS-Sud, MathÉmatiques, BÂt. 425, 91405 Orsay Cedex, France

E-mail address: laurent.lafforgue@math.u-psud.fr 